TRANSACTIONS OF THE

AMERICAN MATHEMATICAL SOCIETY

Volume 351, Number 4, April 1999, Pages 1605-1661

S $0002-9947(99) 02111-X$

\title{
HARDY SPACES, BMO, AND BOUNDARY VALUE PROBLEMS FOR THE LAPLACIAN ON A SMOOTH DOMAIN IN $\mathbf{R}^{N}$
}

\author{
DER-CHEN CHANG, GALIA DAFNI, AND ELIAS M. STEIN
}

\begin{abstract}
We study two different local $H^{p}$ spaces, $0<p \leq 1$, on a smooth domain in $\mathbf{R}^{n}$, by means of maximal functions and atomic decomposition. We prove the regularity in these spaces, as well as in the corresponding dual BMO spaces, of the Dirichlet and Neumann problems for the Laplacian.
\end{abstract}

\section{INTRODUCTION}

Let $\Omega$ be a bounded domain in $\mathbf{R}^{n}$, with smooth boundary. The $L^{p}$ regularity of elliptic boundary value problems on $\Omega$, for $1<p<\infty$, is a classical result in the theory of partial differential equations (see e.g. [ADN]). In the situation of the whole space without boundary, i.e. where $\Omega$ is replaced by $\mathbf{R}^{n}$, the results for $L^{p}$, $1<p<\infty$, extend to the Hardy spaces $H^{p}$ when $0<p \leq 1$ and to BMO. Thus it is a natural question to ask whether the $L^{p}$ regularity of elliptic boundary value problems on a domain $\Omega$ has an $H^{p}$ and BMO analogue, and what are the $H^{p}$ and BMO spaces for which it holds.

This question was previously studied in [CKS], where partial results were obtained and were framed in terms of a pair of spaces, $h_{r}^{p}(\Omega)$ and $h_{z}^{p}(\Omega)$. These spaces, variants of those defined in $[\mathrm{M}]$ and [JSW], are, roughly speaking, the "largest" and "smallest" $h^{p}$ spaces that can be associated to a domain $\Omega$.

Our purpose here is to substantially extend the previous results by determining those $h^{p}$ spaces on $\Omega$ which are particularly applicable to boundary value problems. These spaces allow one to prove sharp results (preservation of the appropriate $h^{p}$ spaces) for all values of $p, 0<p \leq 1$, as well as the preservation of corresponding spaces of BMO functions.

0.1. Motivation and statement of results. There are two approaches to defining the appropriate Hardy spaces on $\Omega$. Recall that the spaces $H^{p}\left(\mathbf{R}^{n}\right)$, for $p<1$, are spaces of distributions. Thus one approach is to look at the problem from the point of view of distributions on $\Omega$. If we denote by $\mathcal{D}(\Omega)$ the space of smooth functions with compact support in $\Omega$, and by $\mathcal{D}^{\prime}(\Omega)$ its dual, we can consider the space of distributions in $\mathcal{D}^{\prime}(\Omega)$ which are the restriction to $\Omega$ of distributions in $H^{p}\left(\mathbf{R}^{n}\right)$ (or in $h^{p}\left(\mathbf{R}^{n}\right)$, the local Hardy spaces defined in [G].) These spaces were studied in $[\mathrm{M}]$ (for arbitrary open sets) and in [CKS] (for Lipschitz domains), where they were denoted $h_{r}^{p}(\Omega)$ (the $r$ stands for "restriction".)

While one is able to prove regularity results for the Dirichlet problem for these spaces when $p$ is near 1 (see [CKS]), these spaces are no longer appropriate when $p$

Received by the editors September 5, 1996 and, in revised form, March 20, 1997.

1991 Mathematics Subject Classification. Primary 35J25, 42B25; Secondary 46E15, 42B30.

(C)1999 American Mathematical Society 
is small, nor for the Neumann problem. This is illustrated for the Dirichlet problem by the following example. Let $x$ be a point on $\partial \Omega$, and denote by $f$ the distribution which is the normal derivative of the delta function at $x$. Such a distribution is in the local Hardy space $h^{p}\left(\mathbf{R}^{n}\right)$ when $p<\frac{n}{n+1}$. Furthermore, it is possible to take a sequence of $L^{2}$ functions $a_{j}$ (if fact $h_{r}^{p}$ atoms) such that $a_{j} \rightarrow f$ as distributions. Since $f$ vanishes on $\Omega$, this means $a_{j} \rightarrow 0$ in $\mathcal{D}^{\prime}(\Omega)$. Now consider the Dirichlet problem for the Laplacian on $\Omega$, defined for smooth functions $\varphi$ by

$$
\begin{gathered}
\Delta u=\varphi \text { on } \Omega, \\
u=0 \text { on } \partial \Omega .
\end{gathered}
$$

Let $\mathbf{G}$ be Green's operator for the Dirichlet problem, i.e. $u=\mathbf{G}(\varphi)$. By the $L^{2}$ theory, we can solve this problem for each $a_{j}$, and since $\mathbf{G}$ is self-adjoint, we have, for every $\varphi \in \mathcal{D}(\Omega)$,

$$
\left\langle\mathbf{G}\left(a_{j}\right), \varphi\right\rangle=\left.\left\langle a_{j}, \mathbf{G}(\varphi)\right\rangle \rightarrow \frac{\partial}{\partial \vec{n}} \mathbf{G}(\varphi)\right|_{x}
$$

as $j \rightarrow \infty$. Note that for the Dirichlet problem, the normal derivative of the solution need not vanish on the boundary. Thus as distributions in $\mathcal{D}^{\prime}(\Omega), \mathbf{G}\left(a_{j}\right) \not \rightarrow 0$. This shows that the problem is not well-defined in $\mathcal{D}^{\prime}(\Omega)$. In essence, this is because the space of test functions, $\mathcal{D}(\Omega)$, is not preserved by the solution of the Dirichlet problem.

To remedy this situation, and define a space of distributions appropriate to the Dirichlet problem, we change our space of test functions from $\mathcal{D}(\Omega)$ to $\mathcal{C}_{d}^{\infty}(\bar{\Omega})$, consisting of functions $\varphi \in \mathcal{C}^{\infty}(\bar{\Omega})$ with $\left.\varphi\right|_{\partial \Omega}=0$ (the $d$ stands for Dirichlet). Note that this space is preserved under the solution to the Dirichlet problem. Thus if we let $\mathcal{C}_{d}^{\infty}(\bar{\Omega})$ be the dual space, we can define the solution to the Dirichlet problem for an element $f$ of $\mathcal{C}_{d}^{\infty \prime}(\bar{\Omega})$ in the sense of distributions. Moreover, if $f$ happens to be a function which is smooth up to the boundary, or a function in $L^{p}$, this solution agrees with $\mathbf{G}(f)$.

We then define the Hardy spaces $h_{d}^{p}(\bar{\Omega})$ to consist of those elements of $\mathcal{C}_{d}^{\infty \prime}(\bar{\Omega})$ satisfying the expected maximal function conditions; here the maximal functions are fashioned out of test functions taken from $\mathcal{C}_{d}^{\infty}(\bar{\Omega})$. For these spaces we get the following regularity result:

Result 0.1. The operators $\frac{\partial^{2} \mathbf{G}}{\partial x_{j} \partial x_{l}}$, defined in the sense of distributions, are bounded from $h_{d}^{p}(\bar{\Omega})$ to $h_{d}^{p}(\bar{\Omega})$, for all $p, 0<p \leq 1$.

This is proved by means of an atomic decomposition for elements of $h_{d}^{p}(\bar{\Omega})$, where atoms supported near the boundary are required to satisfy fewer cancellation conditions than those supported away from the boundary. From this atomic decomposition it can be seen that $h_{d}^{p}(\bar{\Omega})$ is the same as $h_{r}^{p}(\Omega)$ when $\frac{n}{n+1}<p \leq 1$; hence the regularity result is an extension to small $p$ of the $h_{r}^{p}(\Omega)$ regularity result in [CKS].

A second approach to defining Hardy spaces on $\Omega$ is to consider the closure of $\Omega, \bar{\Omega}$, and the distributions in $h^{p}\left(\mathbf{R}^{n}\right)$ which are supported on $\bar{\Omega}$. We shall call the spaces formed by these distributions $h_{z}^{p}(\bar{\Omega})$, where the $z$ denotes the fact that these distributions are zero outside $\bar{\Omega}$. These spaces are the same as those defined in [JSW] (for certain closed sets). A variant of these spaces, $h_{z}^{p}(\Omega)$, formed by taking a quotient of $h_{z}^{p}(\bar{\Omega})$ in order to make it a subspace of $\mathcal{D}^{\prime}(\Omega)$, was defined in [CKS] 
(for Lipschitz domains). By the same reasoning used in the example above, one sees that this quotient space is not appropriate for small $p$ because it eliminates all distributions supported on the boundary.

The spaces $h_{z}^{p}(\bar{\Omega})$ are useful because elements of $h_{z}^{p}(\bar{\Omega})$ have an atomic decomposition into $h^{p}$ atoms supported in $\bar{\Omega}$. Moreover, they are applicable to both the Dirichlet and Neumann problems. Using the atomic decomposition, we can define the operators $\frac{\partial^{2} \mathbf{G}}{\partial x_{j} \partial x_{l}}$ and $\frac{\partial^{2} \widetilde{\mathbf{G}}}{\partial x_{j} \partial x_{l}}$ (where $\widetilde{\mathbf{G}}$ is the solution operator of the Neumann problem for the Laplacian) on $h_{z}^{p}(\bar{\Omega})$, and prove the following regularity result:

Result 0.2. The operators $\frac{\partial^{2} \mathbf{G}}{\partial x_{j} \partial x_{l}}$ and $\frac{\partial^{2} \widetilde{\mathbf{G}}}{\partial x_{j} \partial x_{l}}$ extend to bounded operators from $h_{z}^{p}(\bar{\Omega})$ to $h_{z}^{p}(\bar{\Omega})$, for all $0<p \leq 1$.

A weaker version of this result, namely the boundedness from $h_{z}^{p}(\Omega)$ to $h_{r}^{p}(\Omega)$, is in [CKS]. Note, however, that while the proof given there is valid for atoms, it does not hold for the quotient space $h_{z}^{p}(\Omega)$, since the quotient space norm may be much smaller than the one given by the atomic decomposition.

Once we have the appropriate definitions and regularity results for the $H^{p}$ spaces, when $p=1$, we can consider the corresponding dual BMO spaces. In this case, the dual spaces to $h_{d}^{1}(\bar{\Omega})$ and $h_{z}^{1}(\bar{\Omega})$ are the spaces $\operatorname{bmo}_{z}(\Omega)$ and $\operatorname{bmo}_{r}(\Omega)$, defined in $[\mathrm{M}],[\mathrm{JSW}]$ and $[\mathrm{C}]$. Using some additional arguments, one can convert the $h_{d}^{1}$ and $h_{z}^{1}$ regularity results to the following:

Result 0.3. The operators $\frac{\partial^{2} \mathbf{G}}{\partial x_{j} \partial x_{l}}$ are bounded on $\operatorname{bmo}_{z}(\Omega)$ and on $\mathrm{bmo}_{r}(\Omega)$. Furthermore, the operators $\frac{\partial^{2} \widetilde{\mathbf{G}}}{\partial x_{j} \partial x_{l}}$ are bounded on $\operatorname{bmo}_{r}(\Omega)$.

We should remark that while the results in this paper are stated only for the Laplacian, one can generalize the proofs to any second order elliptic operator, given that the same kind of estimates hold for the various Green's operators.

0.2 . Organization of the paper. In Section 1 , we define the spaces $h_{d}^{p}(\bar{\Omega})$ and $h_{z}^{p}(\bar{\Omega})$. The atomic decompositions for these spaces are given in Section 2. The proof of the atomic decomposition for $h_{d}^{p}(\bar{\Omega})$ uses the maximal function definition and follows the lines of the proof given in [S2] of the atomic decomposition for $H^{p}\left(\mathbf{R}^{n}\right)$.

In Section 3 we prove the $h_{d}^{p}$ regularity of the Dirichlet problem, and in Section 4 we prove the $h_{z}^{p}$ regularity of the Dirichlet and Neumann problems. Besides the atomic decompositions, the proofs of both results make use of the Sobolev estimates for $\mathbf{G}$ and $\widetilde{\mathbf{G}}$, and some Calderón-Zygmund type estimates on the kernels of these operators and their derivatives.

Section 5 contains a different proof of the regularity results when $p=1$, which gives the atomic decomposition directly from a cancellation property inside the domain, without using the maximal function. We then use the $h^{1}$ regularity to prove the bmo regularity. This requires an additional argument involving commutations of vector fields with the Green's operators $\mathbf{G}$ and $\tilde{\mathbf{G}}$.

In the last section we turn from regularity problems to some more analysis of the Hardy spaces themselves. The results in Section 6 illustrate the various relations among the spaces $h_{d}^{p}(\bar{\Omega}), h_{z}^{p}(\bar{\Omega})$, and the spaces $h_{r}^{p}(\Omega)$ and $h_{z}^{p}(\Omega)$ defined in [CKS]. 


\section{Definition OF SPACES}

Let $\Omega$ be a bounded domain in $\mathbf{R}^{n}$ with smooth boundary. In this section we will define two Hardy spaces on $\Omega$.

We first recall the definition of the local Hardy spaces $h^{p}\left(\mathbf{R}^{n}\right)$, introduced by Goldberg (see $[\mathrm{G}]$ ). One can define these spaces by means of a "grand" maximal function. We call a smooth function $\phi$ on $\mathbf{R}^{n}$ a normalized bump function if it is supported in a ball $B$ of radius $R$, and

$$
\left|\partial^{\alpha} \phi\right| \leq R^{-n-|\alpha|}
$$

for all $|\alpha| \leq N_{p}+1$. Here $N_{p}=[n(1 / p-1)]$, the greatest non-negative integer in $n(1 / p-1)$.

Definition 1.1. For $f \in \mathcal{S}^{\prime}\left(\mathbf{R}^{n}\right)$, define the local grand maximal function $m(f)$ at a point $x \in \mathbf{R}^{n}$ by

$$
m(f)(x)=\sup |\langle f, \varphi\rangle|,
$$

where the supremum is taken over all normalized bump functions supported in balls of radii $R \leq 1$ containing $x$.

For $0<p<\infty$, the space $h^{p}\left(\mathbf{R}^{n}\right)$ is defined as the space of tempered distributions $f \in \mathcal{S}^{\prime}\left(\mathbf{R}^{n}\right)$ for which $m(f) \in L^{p}\left(\mathbf{R}^{n}\right)$, with

$$
\|f\|_{h^{p}\left(\mathbf{R}^{n}\right)} \stackrel{\text { def }}{=}\|m(f)\|_{L^{p}\left(\mathbf{R}^{n}\right)} .
$$

We now restrict ourselves to $p \leq 1$, and consider a subspace of $h^{p}\left(\mathbf{R}^{n}\right)$ specific to $\Omega$.

Definition 1.2. For $0<p \leq 1$, the space $h_{z}^{p}(\bar{\Omega})$ is defined to be the subspace of $h^{p}\left(\mathbf{R}^{n}\right)$ consisting of those elements which are supported on $\bar{\Omega}$, i.e.

$$
h_{z}^{p}(\bar{\Omega})=\left\{f \in h^{p}\left(\mathbf{R}^{n}\right): f=0 \text { on } \mathbf{R}^{n} \backslash \bar{\Omega}\right\},
$$

with

$$
\|f\|_{h_{z}^{p}(\bar{\Omega})} \stackrel{\text { def }}{=}\|f\|_{h^{p}\left(\mathbf{R}^{n}\right)} .
$$

Remarks. 1. This is a special case of the space $h^{p}(F)$ considered in [JSW] for a closed " $d$-set" $F$ satisfying the Markov property (here $F=\bar{\Omega}, d=n$ and $\mu$ is just the restriction of Lebesgue measure to $\bar{\Omega})$.

2. For $p<1$, this is not the same as the space $h_{z}^{p}(\Omega)$ introduced in [CKS], since that space is the quotient space

$$
h_{z}^{p}(\Omega)=h_{z}^{p}(\bar{\Omega}) /\left\{f \in h_{z}^{p}(\bar{\Omega}): f=0 \text { on } \Omega\right\},
$$

consisting of those distributions on $\Omega$ which have extensions to elements of $h_{z}^{p}(\bar{\Omega})$. See Section 6 for more discussion of this space.

3. Following the maximal function definition of $h^{p}\left(\mathbf{R}^{n}\right)$, one can also define $h_{z}^{p}(\bar{\Omega})$ by means of a grand maximal function (see [JSW], for example). In that case, the space of test functions is $\mathcal{C}^{\infty}(\bar{\Omega})$, i.e. smooth functions up to the boundary, and the elements of $h_{z}^{p}(\bar{\Omega})$ can be considered as linear functionals on that space.

For the second space, $h_{d}^{p}(\bar{\Omega})$, we want to restrict our space of test functions to those smooth functions on $\bar{\Omega}$ which vanish on $\partial \Omega$. Denoting this space by $\mathcal{C}_{d}^{\infty}(\bar{\Omega})$, we have

$$
\mathcal{C}_{d}^{\infty}(\bar{\Omega})=\left\{\varphi \in \mathcal{C}^{\infty}(\bar{\Omega}):\left.\varphi\right|_{\partial \Omega}=0\right\}
$$


We take the topology of $\mathcal{C}_{d}^{\infty}(\bar{\Omega})$ to be that inherited from $\mathcal{C}^{\infty}(\bar{\Omega})$, and let $\mathcal{C}_{d}^{\infty}(\bar{\Omega})$ denote the dual space.

We call a function $\varphi \in \mathcal{C}_{d}^{\infty}$ a normalized $\mathcal{C}_{d}^{\infty}$ bump function if it is the restriction to $\bar{\Omega}$ of a normalized bump function $\phi$ on $\mathbf{R}^{n}$, with $\left.\phi\right|_{\partial \Omega}=0$.

Definition 1.3. For $f \in \mathcal{C}_{d}^{\infty}(\bar{\Omega})$, define the maximal function $m_{d}(f)$ at a point $x$ in $\Omega$ by

$$
m_{d}(f)(x)=\sup |\langle f, \varphi\rangle|,
$$

where the supremum is taken over all normalized $\mathcal{C}_{d}^{\infty}$ bump functions supported in balls of radii $R \leq 1$ containing $x$.

For $0<p \leq 1$, set

$$
h_{d}^{p}(\bar{\Omega})=\left\{f \in \mathcal{C}_{d}^{\infty \prime}(\bar{\Omega}): m_{d}(f) \in L^{p}(\Omega)\right\},
$$

and for $f \in h_{d}^{p}(\bar{\Omega})$,

$$
\|f\|_{h_{d}^{p}(\bar{\Omega})} \stackrel{\text { def }}{=}\left\|m_{d}(f)\right\|_{L^{p}(\Omega)} .
$$

Remarks. 1. In the definition of the maximal function $m_{d}$, we could have taken the supremum over all normalized $\mathcal{C}_{d}^{\infty}$ bump functions supported in balls of radii $R \leq \delta$, for some fixed $\delta>0$. This new maximal function, $m_{d}^{\delta}$, is equivalent to $m_{d}$ in the sense that they define the same space $h_{d}^{p}$, although the norms differ by a constant depending on $\delta$.

2. Convergence in the " $h_{d}^{p}(\bar{\Omega})$-norm" implies convergence in $\mathcal{C}_{d}^{\infty \prime}(\Omega)$. To see this, it suffices to show that for $f \in h_{d}^{p}(\bar{\Omega})$ and $\varphi \in \mathcal{C}_{d}^{\infty}(\bar{\Omega})$,

$$
|\langle f, \varphi\rangle| \leq C_{\varphi}\|f\|_{h_{d}^{p}(\bar{\Omega})},
$$

where the constant may depend on $\varphi$ but not on $f$.

\section{Atomic DeComposition}

We want to have a characterization of our spaces by means of atomic decomposition. We begin by giving a variant of the definition of atoms for the local Hardy spaces $h^{p}\left(\mathbf{R}^{n}\right)$.

Notation. In what follows, the word "cube" shall mean a cube with sides parallel to the axes, and if $A$ is a constant, $A Q$ shall denote the dilated cube, meaning the cube with the same center as $Q$ with sidelength multiplied by $A$.

Definition 2.1. Let $0<p \leq 1, N_{p}=[n(1 / p-1)]$, and $\nu_{p}=\left(N_{p}+1\right) / n+1-1 / p$. A function $a$ will be called an $h^{p}\left(\mathbf{R}^{n}\right)$ atom if it is supported in a cube $Q \subset \mathbf{R}^{n}$ and satisfies the size condition

$$
\|a\|_{\infty} \leq|Q|^{-1 / p}
$$

and, when $|Q|<1$, the approximate moment conditions

$$
\left|\int a(x)\left(x-x_{Q}\right)^{\alpha} d x\right| \leq|Q|^{\nu_{p}}
$$

for all multi-indices $\alpha$ with $|\alpha| \leq N_{p}$. Here $x_{Q}$ is the center of the cube $Q$.

Remarks. 1. Atoms supported in cubes $Q$ with $|Q|<1$ and satisfying zero moment conditions up to order $N_{p}$ (i.e. $h^{p}$ atoms as defined in [G]) satisfy conditions 2.2 trivially for $|\alpha| \leq N_{p}$. Conversely, as shown in [D], an atom $a$ which satisfies conditions 2.2 for any $\nu>0$ (not necessarily $\nu_{p}$ ) is in $h^{p}\left(\mathbf{R}^{n}\right)$ 
with $\|a\|_{h^{p}} \leq C_{\nu}$. Thus a distribution $f \in \mathcal{S}^{\prime}\left(\mathbf{R}^{n}\right)$ is in $h^{p}\left(\mathbf{R}^{n}\right)$ if for some $\nu>0$ it can be decomposed as $\sum \lambda_{j} a_{j}$, where $\sum\left|\lambda_{j}\right|^{p}<\infty$ and the $a_{j}$ are atoms satisfying conditions 2.2 for that $\nu$. This shows that the atomic spaces corresponding to different choices of $\nu$ in conditions 2.2 are in fact all equivalent to $h^{p}\left(\mathbf{R}^{n}\right)$, except for the dependence of the norm on $\nu$. As will be seen below, it is most convenient to take $\nu=\nu_{p}=\left(N_{p}+1\right) / n-1 / p+1$.

2. Due to the choice of $\nu_{p}$, the size condition for an $h^{p}\left(\mathbf{R}^{n}\right)$ atom $a$ automatically gives conditions 2.2 for moments of order $|\alpha|=N_{p}+1$, since

$$
\left|\int a(x)\left(x-x_{Q}\right)^{\alpha} d x\right| \leq(\operatorname{diam}(Q))^{N_{p}+1}|Q|^{-1 / p+1}=C_{n, p}|Q|^{\nu_{p}} .
$$

By expanding any $\psi \in \mathcal{C}^{\infty}$ in a Taylor expansion of order $N_{p}$ around $x_{Q}$, we can use conditions 2.2 to get

$$
\left|\int a(x) \psi(x) d x\right| \leq C_{n, p}\|\psi\|_{\mathcal{C}^{N_{p}+1}(Q)}|Q|^{\nu_{p}}
$$

where

$$
\|\psi\|_{\mathcal{C}^{N}(Q)}=\sum_{|\alpha| \leq N} \sup _{x \in Q} \frac{1}{|\alpha| !}\left|\psi^{(\alpha)}(x)\right| .
$$

Requiring this for all $\psi \in \mathcal{C}^{\infty}$ is in fact equivalent to the moment conditions 2.2 .

3. Remark 2 show that the conditions imposed on an atom $a$ are invariant under a smooth change of coordinates, in the following sense: if we make a smooth change of variables $y=\Phi(x)$, set $\tilde{a}(y)=a\left(\Phi^{-1}(y)\right)$, and let $\widetilde{Q}$ be the smallest cube containing $\Phi(Q)$, we have

$$
\|\tilde{a}\|_{\infty} \leq C_{\Phi}|\widetilde{Q}|^{-1 / p}
$$

and

$$
\left|\int \tilde{a}(y)\left(y-y_{\tilde{Q}}\right)^{\alpha} d y\right| \leq C_{\Phi}|\widetilde{Q}|^{\nu_{p}}
$$

where the constant $C_{\Phi}$ depends on $\Phi$ and its derivatives up to order $N_{p}+1$. This follows from Remark 2 by letting $\psi(x)=\left(\Phi(x)-y_{\widetilde{Q}}\right)^{\alpha}\left|J_{\Phi}(x)\right|$.

We now define some atoms specific to the domain $\Omega$. First:

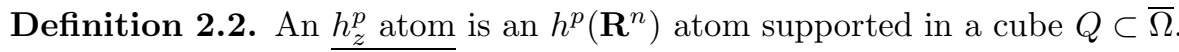

Next, we turn to $h_{d}^{p}$ atoms and define two types of atoms, depending on the position of their supporting cubes with respect to the domain. This definition uses some constants arising from the geometry of the domain.

Definition 2.3. Let $A_{\Omega}, B_{\Omega}$, and $C_{\Omega}$ be constants, with $A_{\Omega}>1, B_{\Omega}>0$, and $C_{\Omega}>0$.

A cube will be called a type (a) cube if the dilated cube $A_{\Omega} Q$ is contained in $\Omega$. A function $a$ will be called a type (a) $h_{d}^{p}$ atom if it is an $h^{p}\left(\mathbf{R}^{n}\right)$ atom supported in a type (a) cube, with the modification that it need only satisfy the approximate moment conditions 2.2 when $|Q|<C_{\Omega}$.

A cube will be called a type (b) cube if

$$
A_{\Omega} Q \cap \partial \Omega \neq \emptyset
$$


but

$$
|Q \cap \bar{\Omega}|>B_{\Omega}|Q|
$$

A function $a$ will be called a type (b) $h_{d}^{p}$ atom if it is supported in a type (b) cube, satisfies the size condition $\overline{2.1 \text {, and, when }|Q|}<C_{\Omega}$, the approximate moment conditions

$$
\left|\int a(x) \psi(x) d x\right| \leq\|\psi\|_{\mathcal{C}^{N_{p}+1}(Q)}|Q|^{\nu_{p}}
$$

for all $\psi \in \mathcal{C}_{d}^{\infty}(\bar{\Omega})$.

Remarks. 1. The conditions on a type (a) cube guarantee that the cube is "far away" from the boundary, relative to its size. Thus type (a) $h_{d}^{p}$ atoms will be invariant under diffeomorphisms of $\Omega$, in the sense of the remarks above, with the possible change of the constants $A_{\Omega}$ and $C_{\Omega}$.

If in addition the diffeomorphism can be extended to a neighborhood of $\bar{\Omega}$, then type (b) $h_{d}^{p}$ atoms will also be invariant, again up to a change of constants.

2. The conditions on a type (b) cube guarantee that the cube is "near" the boundary relative to its size, and that a significant portion of the cube is inside the domain. We do not require that the cube be completely included in the domain because we want to allow type (b) $h_{d}^{p}$ atoms to be supported up to the boundary; hence their supporting cubes cannot be inside the domain unless the boundary is flat and parallel to the axes. We will see, however, that we can assume this locally under an appropriate change of variables.

3. Using the constants $B_{\Omega}$ and $C_{\Omega}$, one may define a modified version of $h_{z}^{p}$ atoms. We will call a function $a$ a modified $h_{z}^{p}$ atom if it is supported in a cube $Q$ with $|Q \cap \bar{\Omega}|>B_{\Omega}|Q|$, satisfies the size condition 2.1 , and satisfies the approximate moment conditions 2.2 when $|Q|<C_{\Omega}$. In fact, this is exactly a type (a) or a type (b) $h_{d}^{p}$ atom with full moment conditions. Unlike the standard $h_{z}^{p}$ atoms, these atoms are invariant under a diffeomorphism of $\bar{\Omega}$, up to a change of constants.

Note that the atoms we have defined indeed belong to the appropriate spaces, with uniformly bounded norms. This is obviously true for $h_{z}^{p}$ atoms (including the modified version), since they are in $h^{p}\left(\mathbf{R}^{n}\right)$ with bounded norm, and vanish outside $\bar{\Omega}$.

For $h_{d}^{p}$ atoms, we have the following

Lemma 2.4. If $a$ is an $h_{d}^{p}$ atom (of either type), then $a \in h_{d}^{p}(\bar{\Omega})$ and

$$
\|a\|_{h_{d}^{p}(\bar{\Omega})}=\left\|m_{d}(a)\right\|_{L^{p}(\Omega)} \leq C_{p},
$$

where $C_{p}$ is independent of $a$.

The proof follows the standard arguments and so is omitted.

We now recall the atomic decomposition in $h_{z}^{p}(\bar{\Omega})$.

Theorem 2.5 ([JSW], [CKS]). Let $\Omega$ be a bounded domain in $\mathbf{R}^{n}$, with $\mathcal{C}^{\infty}$ boundary, and $0<p \leq 1$. A distribution $f \in \mathcal{S}^{\prime}\left(\mathbf{R}^{n}\right)$ is in $h_{z}^{p}(\bar{\Omega})$ if and only if it has a decomposition

$$
f=\sum \lambda_{l} a_{l}
$$


in $\mathcal{S}^{\prime}\left(\mathbf{R}^{n}\right)$, where the $a_{l}$ are $h_{z}^{p}$ atoms and the $\lambda_{l}$ are complex numbers satisfying

$$
\sum\left|\lambda_{l}\right|^{p}<\infty
$$

Furthermore,

$$
\|f\|_{h_{z}^{p}}^{p} \approx \inf \left(\sum\left|\lambda_{l}\right|^{p}\right)
$$

where the infimum is taken over all such decompositions, and the constants of proportionality are independent of $f$.

Remarks. 1. Jonsson, Sjögren, and Wallin (see [JSW], Theorems 3.1 and 3.2) prove this decomposition in the case of a closed " $d$-set" $F$ in $\mathbf{R}^{n}$ satisfying the Markov property (see Remark 1 following Definition 1.2). In their proof, the $h_{z}^{p}$ atoms are actually the modified $h_{z}^{p}$ atoms (see Remark 3 above), i.e. they are supported in $F$, and their supports are contained in balls with centers in $F$, but the balls need not be contained in $F$.

2. In ([CKS], Theorem 3.2), this decomposition is stated for a bounded Lipschitz domain $\Omega$, not for the space $h_{z}^{p}(\bar{\Omega})$, but for the quotient space $h_{z}^{p}(\Omega)$, without the bounds on the norms. However, the proof (via the square function) only assumes that $f \in h^{p}\left(\mathbf{R}^{n}\right)$ and $f$ is supported in $\bar{\Omega}$, so it in fact holds for $f \in h_{z}^{p}(\bar{\Omega})$, and in that case one gets the bounds on the norms by noting that the $h_{z}^{p}(\bar{\Omega})$ norm of $f$ is the same as its $h^{p}\left(\mathbf{R}^{n}\right)$ norm, which bounds the $L^{p}$ norm of the square function, and which in turn bounds $\left(\sum\left|\lambda_{l}\right|^{p}\right)^{1 / p}$. Moreover, by a slight modification of their argument it can be shown that all the atoms may be taken to be of type (a), i.e. with support $Q$ such that $A_{\Omega} Q$ is still in $\Omega$. It should be added that at the time the paper [CKS] was written, the authors were unfortunately unaware of the earlier work of [JSW].

We are now ready to state the main result of this section.

Theorem 2.6. Let $\Omega$ be a bounded domain in $\mathbf{R}^{n}$, with $\mathcal{C}^{\infty}$ boundary. Then there are constants $A_{\Omega}, B_{\Omega}$, and $C_{\Omega}$ (as in Definition 2.3) such that the following holds:

$A$ distribution $f \in \mathcal{C}_{d}^{\infty \prime}(\bar{\Omega})$ is in $h_{d}^{p}(\bar{\Omega})$ if and only if it has a decomposition

$$
f=\sum \lambda_{l} a_{l}+\sum \mu_{m} a_{m}
$$

in $\mathcal{C}_{d}^{\infty \prime}$, where the first sum is taken over type (a) $h_{d}^{p}$ atoms, the second sum is taken over type $(\mathrm{b}) h_{d}^{p}$ atoms, and $\lambda_{l}, \mu_{m}$ are complex numbers satisfying

$$
\sum\left|\lambda_{l}\right|^{p}+\sum\left|\mu_{m}\right|^{p}<\infty
$$

Furthermore,

$$
\|f\|_{h_{d}^{p}}^{p} \approx \inf \left(\sum\left|\lambda_{l}\right|^{p}+\sum\left|\mu_{m}\right|^{p}\right)
$$

where the infimum is taken over all such decompositions, and the constants of proportionality are independent of $f$.

Proof. The easy part of the proof is the "if" part, that is, assuming $f$ has such a decomposition in $\mathcal{C}_{d}^{\infty}(\Omega)$. For then, if the sum is finite,

$$
\langle f, \varphi\rangle=\sum \lambda_{l}\left\langle a_{l}, \varphi\right\rangle+\sum \mu_{m}\left\langle a_{m}, \varphi\right\rangle
$$

for all normalized $\mathcal{C}_{d}^{\infty}$ bump functions $\varphi$, and so

$$
m_{d}(f)(x) \leq \sum\left|\lambda_{l}\right| m_{d}\left(a_{l}\right)(x)+\sum\left|\mu_{m}\right| m_{d}\left(a_{m}\right)(x) ;
$$


hence

$$
\begin{aligned}
\left\|m_{d}(f)\right\|_{L^{p}(\Omega)}^{p} & \leq \sum\left|\lambda_{l}\right|^{p}\left\|m_{d}\left(a_{l}\right)\right\|_{L^{p}(\Omega)}^{p}+\sum\left|\mu_{m}\right|^{p}\left\|m_{d}\left(a_{m}\right)\right\|_{L^{p}(\Omega)}^{p} \\
& \leq C_{\nu}^{p}\left(\sum\left|\lambda_{l}\right|^{p}+\sum\left|\mu_{m}\right|^{p}\right)
\end{aligned}
$$

by the lemma. This gives the convergence of the sum in the $h_{d}^{p}$ norm, and so $f \in h_{d}^{p}(\bar{\Omega})$.

As for the "only if" part, let us first state the result for a special case. We will then use this to prove the general case.

Lemma 2.7. Let $D$ be a smoothly bounded domain contained in the upper halfspace $\mathbf{R}_{+}^{n}$ and containing the open upper half-ball $B_{+}(0,10)=B(0,10) \cap \mathbf{R}_{+}^{n}$. Suppose $f \in h_{d}^{p}(\bar{D})$ and $f$ is supported in $B(0,1)$. Then $f$ has a decomposition

$$
f=\sum \lambda_{i} a_{i}
$$

in $\mathcal{C}_{d}^{\infty}(\bar{D})$. Here the $\lambda_{i}$ are complex numbers satisfying

$$
C_{1}\|f\|_{h_{d}^{p}(\bar{D})}^{p} \leq \sum\left|\lambda_{i}\right|^{p} \leq C_{2}\|f\|_{h_{d}^{p}(\bar{D})}^{p}
$$

for some constants $C_{1}$ and $C_{2}$ depending only on $p$, and the $a_{i}$ are as follows.

Each $a_{i}$ is a function supported in a cube $Q_{i}$ which is contained in the half-open upper half-ball $B(0,3) \cap \overline{\mathbf{R}_{+}^{n}}$, and

$$
\left\|a_{i}\right\|_{\infty} \leq\left\|Q_{i}\right\|^{-1 / p}
$$

Furthermore, there exists a constant $C_{n}$ (depending only on the dimension) such that when $\left|Q_{i}\right|<C_{n}, a_{i}$ satisfies the following cancellation conditions. If $2 Q_{i} \subset$ $\mathbf{R}_{+}^{n}$, then

$$
\int_{Q_{i}} a_{i}(x) x^{\alpha} d x=0
$$

for all monomials $x^{\alpha}$ of degree $|\alpha| \leq N_{p}$. If $2 Q_{i} \cap \partial \mathbf{R}_{+}^{n} \neq \emptyset$, then

$$
\int_{Q_{i}} a_{i}(x) x^{\alpha} d x=0
$$

for all monomials $x^{\alpha}$ of degree $|\alpha| \leq N_{p}$ with $\alpha_{n} \geq 1$.

Assuming the lemma, we continue with the proof of Theorem 2.6.

We take a partition of unity for $\bar{\Omega}$ as follows. Let $\eta_{j}, j=0, \ldots, k$, be $\mathcal{C}^{\infty}$ functions, $\sum \eta_{j}=1$ on $\bar{\Omega}$. For $j=0, \eta_{0}$ has compact support in $\Omega$. For $1 \leq j \leq k$, $\eta_{j}$ has compact support inside an open set $U_{j}$ with $U_{j} \cap \partial \Omega \neq \emptyset$. Furthermore, each $U_{j}$ is contained in a larger open set $V_{j}$ such that there is a $\mathcal{C}^{\infty}$ diffeomorphism $\Phi_{j}$ between $V_{j}$ and the ball $B(0,10)$ which maps $V_{j} \cap \Omega$ onto $B_{+}(0,10)=B(0,10) \cap \mathbf{R}_{+}^{n}$, $V_{j} \cap \partial \Omega$ into $\partial \mathbf{R}_{+}^{n}$, and $U_{j}$ into $B(0,1)$.

Write $f=\sum\left(\eta_{j} f\right)$. Consider first $j=0$. Set

$$
\delta=\frac{1}{2} \operatorname{dist}\left(\operatorname{supp}\left(\eta_{0}\right), \partial \Omega\right)
$$

and let $m^{\delta}$ be the local grand maximal function defined as in Definition 1.1 but with bump functions supported in balls of diameter bounded by $\delta$. Notice that $\eta_{0} f \in \mathcal{S}^{\prime}$, and

$$
m^{\delta}\left(\eta_{0} f\right)(x)=0
$$


for $x \notin \Omega$. For $x \in \Omega$,

$$
m^{\delta}\left(\eta_{0} f\right)(x) \leq C_{0} m_{d}(f)(x)
$$

where the constant $C_{0}$ only depends on the derivatives of $\eta_{0}$ up to order $N_{p}+1$. Thus

$$
\left\|m^{\delta}\left(\eta_{0} f\right)\right\|_{L^{p}\left(\mathbf{R}^{n}\right)} \leq C_{0}\left\|m_{d}(f)\right\|_{L^{p}(\Omega)}<\infty
$$

so $\eta_{0} f \in h^{p}\left(\mathbf{R}^{n}\right)$.

Since the support of $m^{\delta}\left(\eta_{0} f\right)$ lies inside $\Omega$, and at least distance $\delta$ from the boundary, we can get an atomic decomposition

$$
\eta_{0} f=\sum \lambda_{i} a_{i}
$$

where $a_{i}$ are $h^{p}\left(\mathbf{R}^{n}\right)$ atoms supported in cubes $Q_{i} \subset \Omega$ with $\operatorname{dist}\left(Q_{i}, \partial \Omega\right) \geq \delta$ for all $i$. If $2 Q_{i} \subset \Omega$, then $a_{i}$ is a type (a) $h_{d}^{p}$ atom. If $2 Q_{i} \cap \partial \Omega \neq \emptyset$, then $a_{i}$ is a type (b) $h_{d}^{p}$ atom because $a_{i}$ satisfies the size condition and, when $|Q|<1$, the full moment conditions $a_{i}$ satisfies as an $h^{p}\left(\mathbf{R}^{n}\right)$ atom imply

$$
\left|\int a_{i}(x) \varphi(x) d x\right| \leq\|\varphi\|_{\mathcal{C}^{N_{p}+1}(Q)}\left|Q_{j}\right|^{\nu_{p}}
$$

for all $\varphi \in \mathcal{C}^{\infty}$ (see Remark 2 following Definition 2.1,) hence for all $\varphi \in \mathcal{C}_{d}^{\infty}(\bar{\Omega})$.

Now fix $j, 1 \leq j \leq k$, and write $\eta$ for $\eta_{j}, U$ for $U_{j}, V=V_{j}$, and $\Phi=\Phi_{j}$. Let $\tau=\eta \circ \Phi^{-1}$. Then $\tau$ is a $\mathcal{C}^{\infty}$ function supported in $B(0,1)$. Note that if $\varphi$ is a $\mathcal{C}^{\infty}$ function supported in $\overline{B_{+}(0,10)}$ and $\left.\varphi\right|_{\partial \mathbf{R}_{+}^{n}}=0$, then $\varphi \circ \Phi \in \mathcal{C}_{d}^{\infty}(\Omega)$. Define $g$ acting on such $\varphi$ by

$$
\langle g, \varphi\rangle=\langle\eta f, \varphi \circ \Phi\rangle=\langle f,(\tau \varphi) \circ \Phi\rangle .
$$

If $\varphi$ is supported outside $B(0,1)$, this gives 0 . Thus we can extend $g$ to act on any $\varphi \in \mathcal{C}_{d}^{\infty}(D)$ by setting it to be zero on $D \backslash B(0,1)$. The continuity of $f$ implies that of $g$, so $g \in \mathcal{C}_{d}^{\infty \prime}(D)$ and $g$ is supported in $B(0,1)$.

In order to use the lemma, we want to show $g \in h_{d}^{p}(\bar{D})$. So suppose $x \in B(0,10)$ and $\varphi_{t}^{x}$ is the restriction to $\mathbf{R}_{+}^{n}$ of a normalized $\mathcal{C}^{\infty}$ bump function, supported in a ball radius $t \leq 1$ containing $x$, and vanishing on $\partial \mathbf{R}_{+}^{n}$. Then $\psi=\left(\tau \varphi_{t}^{x}\right) \circ \Phi \in \mathcal{C}_{d}^{\infty}(\bar{\Omega})$ and is supported in a ball of radius $\delta$ containing $\Phi^{-1}(x)$, where $\delta$ depends only on $\Phi$. We can write $\psi$ as a constant multiple of a normalized $\mathcal{C}_{d}^{\infty}$ bump function supported in a ball of radius $\delta$, the constant depending only on $\eta$ and $\Phi$. Thus

$$
\left|\left\langle g, \varphi_{t}^{x}\right\rangle\right|=|\langle f, \psi\rangle| \leq C m_{d}^{\delta}(f)\left(\Phi^{-1}(x)\right)
$$

so by Remark 1 following Definition 1.3,

$$
\left\|m_{d}(g)\right\|_{L^{p}(D)} \leq C\left\|m_{d}(f)\right\|_{L^{p}(\Omega)},
$$

and $g \in h_{d}^{p}(\bar{D})$.

Thus $g$ satisfies the hypotheses of Lemma 2.7, and we get a decomposition

$$
g=\sum \lambda_{i} a_{i}
$$

Let $\widetilde{a_{i}}=a_{i} \circ \Phi$, and let $\widetilde{Q_{i}}$ be the smallest cube containing $\Phi^{-1}\left(Q_{i}\right)$, where $Q_{i}$ is the cube in which $a_{i}$ is supported. We want to show that the $\widetilde{a_{i}}$ are multiples of either type (a) or type (b) atoms.

Since the $a_{i}$ satisfy the size condition, we get that

$$
\left\|\widetilde{a_{i}}\right\|_{\infty}=\left\|a_{i}\right\|_{\infty} \leq\left|Q_{i}\right|^{-1 / p} \leq C_{\Phi, \Omega}\left|\widetilde{Q_{i}}\right|^{-1 / p} .
$$


Since the $Q_{i}$ are all contained in $\overline{B_{+}(0,3)}$, the constant $C_{\Phi, \Omega}$ only depends on the bounds on the derivatives of $\Phi$ in a compact set, as well as on the geometry of $\Omega$ (and of course $p$ and $n$ ). Thus we can divide all the $\widetilde{a_{i}}$ by this constant, and they will satisfy the size condition.

To check the cancellation conditions, we must specify the constants used in Definition 2.3. We will do this first just for the map $\Phi=\Phi_{j}$. There exists a constant $A_{\Phi}>1$ (depending only on the maximum and minimum of the derivatives of $\Phi$ on a compact set) such that for any cube $Q \subset B_{+}(0,3)$, if $\widetilde{Q}$ is the smallest cube containing $\Phi^{-1}(Q)$, and if $A_{\Phi} \widetilde{Q} \subset \Omega$, then $2 Q \subset \mathbf{R}_{+}^{n}$. Furthermore, there exists a constant $B_{\Phi}>0$, again depending only on $\Phi$ and the geometry of $\Omega$, such that if $Q$ is a cube in $\overline{B_{+}(0,3)}$, and $2 Q \cap \overline{\mathbf{R}_{+}^{n}} \neq 0$, then the smallest cube $\widetilde{Q}$ containing $\Phi^{-1}(Q)$ satisfies $|\widetilde{Q} \cap \Omega| \geq B_{\Phi}|\widetilde{Q}|$. Finally, there exists a constant $C_{\Phi}>0$ (in fact a constant multiple of the minimum for the Jacobian determinant $\left|J_{\Phi}\right|$ on $\left.\Phi^{-1}\left(\overline{B_{+}(0,3)}\right)\right)$ such that if $Q$ and $\widetilde{Q}$ are as above, and $|\widetilde{Q}|<C_{\Phi}$, then $|Q|<C_{n}$ (with $C_{n}$ as in Lemma 2.7).

Now note that since there are only finitely many $\Phi_{j}$, we can choose these constants uniformly. (Here we also take into account the implicit choices made for $j=0$, namely $A_{\Phi}=2, B_{\Phi}=1$ and $C_{\Phi}=1$.) Thus we will replace $A_{\Phi}, B_{\Phi}$ and $C_{\Phi}$ by $A_{\Omega}, B_{\Omega}$ and $C_{\Omega}$, respectively. We are now ready to prove the cancellation conditions.

First suppose $A_{\Omega} \widetilde{Q_{i}} \subset \Omega$. Since all the cubes $Q_{i} \subset \overline{B_{+}(0,3)}$, we have, by the choice of $A_{\Omega}$, that $2 Q_{i} \subset \mathbf{R}_{+}^{n}$. In that case $a_{i}$ in an $h^{p}\left(\mathbf{R}^{n}\right)$ atom, so if in addition $\left|\widetilde{Q_{i}}\right|<C_{\Omega}$, then $\left|Q_{i}\right|<C_{n}$, and

$$
\int_{Q_{i}} a_{i}(y) y^{\alpha} d y=0
$$

whenever $|\alpha| \leq N_{p}$. From Remarks 1 and 3 following Definition 2.1, we get

$$
\left|\int_{\widetilde{Q_{i}}} \widetilde{a}_{i}(x)\left(x-x_{\widetilde{Q}_{i}}\right)^{\alpha} d x\right| \leq C\left|\widetilde{Q_{i}}\right|^{\nu_{p}},
$$

where $x_{\widetilde{Q_{i}}}$ is the center of $\widetilde{Q_{i}}$ and the constant $C$ depends on $\Phi$ and the geometry of $\Omega$, but is independent of $\widetilde{a_{i}}$. Dividing $\widetilde{a_{i}}$ by $C$, we get that $\widetilde{a_{i}}$ is a type (a) atom.

Next, suppose $A_{\Omega} \widetilde{Q_{i}} \cap \partial \Omega \neq \emptyset$. Then we have by the choice of $B_{\Omega}$ that

$$
\left|\widetilde{Q_{i}} \cap \bar{\Omega}\right| \geq B_{\Omega}\left|\widetilde{Q_{i}}\right|
$$

So we want to show that $\widetilde{a_{i}}$ satisfies the cancellation conditions for a type (b) $h_{d}^{p}$ atom. Let $\psi \in \mathcal{C}_{d}^{\infty}(\bar{\Omega})$. By using a cut-off function, we may assume $\operatorname{supp}(\psi) \subset V$. Define a function $\varphi$ on $B(0,10) \cap \mathbf{R}_{+}^{n}$ by

$$
\varphi(y)=\psi\left(\Phi^{-1}(y)\right)\left|J_{\Phi}^{-1}(y)\right| .
$$

Then by a change of variables $y=\Phi(x)$, we have

$$
\int_{\widetilde{Q_{i}}}{\widetilde{a_{i}}}_{i}(x) \psi(x) d x=\int_{Q_{i}} a_{i}(y) \varphi(y) d y .
$$

Let $y^{*}$ be the projection of the center of $Q_{i}$ onto $\partial \mathbf{R}_{+}^{n}$. Since $\psi=0$ on $\partial \Omega$, we have that $\varphi=0$ on $\partial \mathbf{R}_{+}^{n}$, so all the tangential derivatives of $\varphi$ at $y^{*}$ vanish. Thus the 
Taylor expansion of $\varphi$ around $y^{*}$ is

$$
\varphi(y)=\sum_{|\alpha| \leq N_{p}, \alpha_{n} \geq 1}\left(\partial^{\alpha} \varphi\right)\left(y^{*}\right)\left(y-y^{*}\right)^{\alpha}+R_{N_{p}+1}\left(\varphi, y, y^{*}\right) .
$$

Plugging this into the integral, we can use the moment conditions on $a_{i}$ (noting that $\left(y-y^{*}\right)^{\alpha}$ contains a factor of $y_{n}$ of order $\left.\alpha_{n} \geq 1\right)$ to get

$$
\begin{aligned}
\left|\int_{Q_{i}} a_{i}(y) \varphi(y) d y\right| & =\left|\int_{Q_{i}} a_{i}(y) R_{N_{p}+1}\left(\varphi, y, y^{*}\right) d y\right| \\
& \leq \sum_{|\alpha|=N_{p}+1} \frac{1}{\left(N_{p}+1\right) !} \sup _{y \in Q_{i}}\left|\partial^{\alpha}(\varphi(y))\right| \int\left|a_{i}(y)\right|\left|y-y^{*}\right|^{\left(N_{p}+1\right)} d y \\
& \leq\|\varphi\|_{\mathcal{C}^{N_{p}+1}\left(Q_{i}\right)}\left|Q_{i}\right|^{1-1 / p} \operatorname{diam}\left(Q_{i}\right)^{\left(N_{p}+1\right)} \\
& \leq C\|\psi\|_{\mathcal{C}^{N_{p}+1}\left(\widetilde{Q_{i}}\right)}\left|\widetilde{Q_{i}}\right|^{\nu_{p}},
\end{aligned}
$$

where the constant $C$ depends on $\Phi$, the cutoff function, and the geometry of $\Omega$, but is independent of $\widetilde{a_{i}}$. Again, we can divide $\widetilde{a_{i}}$ by the constant to get a type (b) $h_{d}^{p}$ atom.

We have thus shown that in $\mathcal{C}_{d}^{\infty}(\bar{\Omega})$,

$$
\eta f=g \circ \Phi=\sum \lambda_{i} a_{i} \circ \Phi=\sum \lambda_{i} \widetilde{a_{i}}=\sum\left(C \lambda_{i}\right)\left(\widetilde{a_{i}} / C\right),
$$

where $\widetilde{a}_{i} / C$ are either type (a) $h_{d}^{p}$ atoms or type (b) $h_{d}^{p}$ atoms. Furthermore,

$$
\sum\left|C \lambda_{i}\right|^{p} \leq C^{\prime}\|g\|_{h_{d}^{p}(\bar{D})}^{p} \leq C^{\prime \prime}\|f\|_{h_{d}^{p}(\bar{\Omega})}^{p} .
$$

Getting back to the global picture, we have, in $\mathcal{C}_{d}^{\infty}(\bar{\Omega})$,

$$
f=\sum_{j=0}^{k} \eta_{j} f=\sum_{j=0}^{k} \sum_{i} \lambda_{i}^{j} a_{i}^{j}
$$

which is an atomic decomposition that satisfies the conditions of the theorem.

In order to prove Lemma 2.7, we will follow the proof in [S2], Chapter III, Section 2 , (which is itself adapted from several earlier arguments going back to the work of Latter and others). We begin with the following proposition.

Proposition 2.8. Let $D$ be as in Lemma 2.7. Suppose that $f \in \mathcal{C}_{d}^{\infty \prime}(\bar{D}), m_{d}(f) \in$ $L^{p}(D)$, and $f$ is supported in $B(0,1)$. Then for $\gamma \geq 0$, there is a decomposition $f=g+b, b=\sum b_{k}$, and a collection of cubes $\left\{Q_{k}^{*}\right\}$, so that

1. the $\left\{Q_{k}^{*}\right\}$ are contained in $B(0,3) \cap \overline{\mathbf{R}_{+}^{n}}$, have the bounded intersection property, and

$$
\text { interior }\left(\bigcup_{k} Q_{k}^{*}\right)=\left\{x \in D: m_{d}(f)(x)>\gamma\right\} ;
$$

2. each $b_{k} \in \mathcal{C}_{d}^{\infty \prime}(\bar{D})$ is supported in $Q_{k}^{*}$ and satisfies

$$
\int_{D} m_{d}\left(b_{k}\right)^{p} d x \leq c \int_{Q_{k}^{*}} m_{d}(f)^{p} d x ;
$$


3. if $Q_{k}^{*}$ is such that $2 Q_{k}^{*} \subset \mathbf{R}_{+}^{n}$, and $\left|Q_{k}^{*}\right|<C_{n}$ (with $C_{n}$ as in Lemma 2.7), then $b_{k}$ satisfies

$$
\left\langle b_{k}, q\right\rangle=0
$$

for all polynomials $q$ of degree up to $N_{p}$; otherwise, if $2 Q_{k}^{*} \cap \partial \mathbf{R}_{+}^{n} \neq \emptyset$, and $\left|Q_{k}^{*}\right|<C_{n}$, then $b_{k}$ satisfies

$$
\left\langle b_{k}, q\right\rangle=0
$$

for all polynomials of degree up to $N_{p}$ which are divisible by $x_{n}$;

4. $g \in \mathcal{C}_{d}^{\infty \prime}(\bar{D})$ and

$$
m_{d}(g) \leq C m_{d}(f)(x) \chi_{F}+C \gamma \sum_{k} \frac{l_{k}^{n+N_{p}+1}}{\left(l_{k}+\left|x-x_{k}\right|\right)^{n+N_{p}+1}},
$$

where $F$ is the closure of the set $\left\{x \in D: m_{d}(f)(x) \leq \gamma\right\}$, and $l_{k}$ and $x_{k}$ are the side-length and center of $Q_{k}^{*}$.

Proof. Let us outline the modifications needed for the proof in [S2], Chapter III, Section 2.2, to apply in this situation.

Let $F$ be the closure of the set $\left\{x \in D: m_{d}(f)(x) \leq \gamma\right\}$ in $\mathbf{R}^{n}$. Note that since $f$ is supported in $B(0,1)$, and the maximal function $m_{d}$ is defined using bump functions supported in balls of radii not greater than 1 , we must have that $m_{d}(f)$ is supported in $B(0,3)$, and hence $(\bar{D} \backslash F) \subset B(0,3) \cap \overline{\mathbf{R}_{+}^{n}}$.

Consider the dyadic Whitney decomposition for $\mathbf{R}^{n} \backslash F$ (see [S1], Ch. VI, Section 1.1). From this decomposition, we take only those cubes lying in $\bar{D}$. This is possible since any cube in the dyadic decomposition lies on one side or the other of $\partial \mathbf{R}_{+}^{n}$, and furthermore $\partial D \subset F \cup \partial \mathbf{R}_{+}^{n}$, so any cube in the decomposition lies either entirely in $\bar{D}$ or entirely outside of it.

Thus we can get closed cubes $Q_{k} \subset B(0,3) \cap \overline{\mathbf{R}_{+}^{n}}$ whose interiors are mutually disjoint, with

$$
\operatorname{diam}\left(Q_{k}\right) \leq \operatorname{dist}\left(Q_{k}, F\right) \leq 4 \operatorname{diam}\left(Q_{k}\right)
$$

and

$$
\bigcup_{k} Q_{k}=\bar{D} \backslash F \subset B(0,3) \cap \overline{\mathbf{R}_{+}^{n}} .
$$

Let $\widetilde{Q_{k}}=\frac{3}{2} Q_{k} \cap \overline{\mathbf{R}_{+}^{n}}$ and $Q_{k}^{*}=2 Q_{k} \cap \overline{\mathbf{R}_{+}^{n}}$. While $Q_{k}^{*}$ may no longer be a cube (in case $\operatorname{dist}\left(Q, \partial \mathbf{R}_{+}^{n}\right)$ is smaller than half the sidelength of $Q$ ), we can enlarge it in the $x_{n}$ direction (by no more than half the sidelength of $Q$ ) to make it a cube. Even if that is the case, we still have that, for all $y \in Q_{k}^{*}$,

$$
\operatorname{dist}\left(y, Q_{k}\right) \leq \frac{\sqrt{n+3}}{2 \sqrt{n}} \operatorname{diam}\left(Q_{k}\right)<\operatorname{diam}\left(Q_{k}\right)
$$

(since $n>1$ ), so as we assumed that $\operatorname{dist}\left(Q_{k}, F\right) \geq \operatorname{diam}\left(Q_{k}\right)$, we have

$$
\operatorname{dist}\left(Q_{k}^{*}, F\right)>0 \text {. }
$$

Combined with the fact that $Q_{k}^{*} \subset \overline{\mathbf{R}_{+}^{n}}$, and recalling that $\partial D \subset F \cup \partial \mathbf{R}_{+}^{n}$, this implies

$$
Q_{k}^{*} \subset \bar{D} \backslash F \subset B(0,3) \cap \overline{\mathbf{R}_{+}^{n}} .
$$

As we already have $\bigcup Q_{k}=\bar{D} \backslash F$, we get that

$$
\bigcup_{k} Q_{k}^{*}=\bar{D} \backslash F
$$


and hence

$$
\text { interior }\left(\bigcup_{k} Q_{k}^{*}\right)=\left\{x \in D: m_{d}(f)(x)>\gamma\right\} \text {. }
$$

In constructing the partition of unity $\left\{\eta_{k}\right\}$ for $\bar{D} \backslash F$, we first follow the construction in [S2], namely we take a partition of unity subordinate to the cubes $\frac{3}{2} Q_{k}$, but then we restrict the functions to $\overline{\mathbf{R}_{+}^{n}}$. Thus $\eta_{k}$ will be smooth when $\widetilde{Q_{k}} \subset \mathbf{R}_{+}^{n}$, and otherwise $\eta_{k}$ will be the restriction of a smooth function to $\overline{\mathbf{R}_{+}^{n}}$.

We set

$$
b_{k}=\left(f-c_{k}\right) \eta_{k},
$$

where the polynomial $c_{k}$ is picked as follows.

Let $C_{n}=(3 \sqrt{n})^{-n}$. If $\left|Q_{k}^{*}\right| \geq C_{n}$, we let $c_{k}=0$.

If $\left|Q_{k}^{*}\right|<C_{n}$ and $Q_{k}^{*}$ is a type (a) cube, namely $2 Q_{k}^{*} \subset \mathbf{R}_{+}^{n}$, then $c_{k}$ is the unique polynomial for which

$$
\left\langle f, q \eta_{k}\right\rangle=\int c_{k} q \eta_{k} d x
$$

for all polynomials $q$ of degree $\leq N_{p}$. Note that we can write

$$
c_{k}(x)=\left\langle f, P(x, \cdot) \eta_{k}\right\rangle
$$

where $P(x, y)$ is the kernel of the projection operator from the $L^{2}$ space with weight function $\eta_{k}$ onto its subspace $\mathcal{H}_{k, N_{p}}$ consisting of polynomials of degree $\leq N_{p}$.

If $\left|Q_{k}^{*}\right|<C_{n}$ and $Q_{k}^{*}$ is a type (b) cube, i.e. $2 Q_{k}^{*} \cap \overline{\mathbf{R}_{+}^{n}} \neq \emptyset$, pick $c_{k}$ to be the unique polynomial for which

$$
\left\langle f, x^{\alpha} \eta_{k}\right\rangle=\int c_{k} x^{\alpha} \eta_{k} d x
$$

for all monomials of degree $|\alpha| \leq N_{p}$ with $\alpha_{n} \geq 1$.

We claim that

and

$$
\left|c_{k} \eta_{k}\right| \leq c \gamma
$$

$$
\left|c_{k} \eta_{k}\right| \leq c m_{d}(f)(x)
$$

for any $x$ in $Q_{k}^{*}$. This is obvious when $\left|Q_{k}^{*}\right| \geq C_{n}$, so assume below that $\left|Q_{k}^{*}\right|<C_{n}$.

For a type (a) cube, this follows from the proof as in [S2].

For type (b) cubes, we can modify the proof by considering instead of $P(x, y)$ the kernel $\widetilde{P}(x, y)$ of the projection onto the subspace of $\mathcal{H}_{k, N_{p}}$ consisting of all polynomials divisible by $x_{n}$. This kernel must vanish for $y \in \partial \mathbf{R}_{+}^{n}$, so $\widetilde{P}(x, y) \eta_{k}(y)$ will also be a normalized $\mathcal{C}_{d}^{\infty}$ bump function in $y$, and the same argument as above shows that it lies in a ball of radius $\leq 1$ containing a point of $F$.

Since $f$ belongs to $\mathcal{C}_{d}^{\infty \prime}(\bar{D})$, so does $b_{k}$, and it is supported in $Q_{k}^{*}$. It remains to show that

$$
\int_{D} m_{d}\left(b_{k}\right)^{p} d x \leq c \int_{Q_{k}^{*}} m_{d}(f)^{p} d x .
$$

We restrict ourselves to "small" cubes, where the main difficulty lies. One notes first that

$$
m_{d}\left(f \eta_{k}\right)(x)=\sup _{\varphi_{t}^{x}, t \leq 1}\left|\left\langle f, \eta_{k} \varphi_{t}^{x}\right\rangle\right| \leq C_{\eta_{k}} m_{d}(f)(x),
$$

and since we already have $\left|c_{k} \eta_{k}\right| \leq C m_{d}(f)(x)$, we get

$$
m_{d}\left(b_{k}\right)(x) \leq C m_{d}(f)(x)
$$


for any $x$ in $Q_{k}^{*}$. Integrating, we get

$$
\int_{Q_{k}^{*}} m_{d}\left(b_{k}\right)^{p} d x \leq C \int_{Q_{k}^{*}} m_{d}(f)^{p} d x .
$$

Looking at $D \backslash Q_{k}^{*}$ and the case when $\left|Q_{k}^{*}\right|<C_{n}$, we can write

$$
\left\langle b_{k}, \varphi_{t}^{x}\right\rangle=\left\langle b_{k}, \varphi_{t}^{x}-q\right\rangle=\left\langle f, \eta_{k}\left(\varphi_{t}^{x}-q\right)\right\rangle .
$$

Here

$$
q(y)=\sum_{|\alpha| \leq N_{p}} \partial^{\alpha} \varphi\left(x_{k}\right)\left(y-x_{k}\right)^{\alpha},
$$

where $x_{k}$ is the center of $Q_{k}^{*}$ if $Q_{k}^{*}$ is a type (a) cube, and the projection of the center of $Q_{k}^{*}$ onto $\partial \mathbf{R}_{+}^{n}$ if $Q_{k}^{*}$ is a type (b) cube. Note that in the latter case, $\partial^{\alpha} \varphi_{t}^{x}\left(x_{k}\right)=0$ if $\alpha_{n}=0$, since $\varphi_{t}^{x}$ vanishes identically on $\partial \mathbf{R}_{+}^{n}$. Thus the only non-zero terms in the sum above involve positive powers of $y_{n}$, against which the integral of $b_{k}$ vanishes.

In both cases, we still have $|x-y| \simeq\left|x-x_{k}\right|$ when $x \notin Q_{k}^{*}$ and $y \in \widetilde{Q_{k}}$, and the radius $t$ of the support of $\varphi_{t}^{x}$ still satisfies $t \geq c\left|x-x_{k}\right|$. Furthermore, $\eta_{k}\left(\varphi_{t}^{x}-q\right)$ is supported in $\widetilde{Q_{k}}$, which (since $\left|Q_{k}^{*}\right|<C_{n}$ ) is contained in a ball of radius $\leq 1$ containing a point of $F$ (see above). Thus the rest of the estimates in [S2] go through unchanged, and we get

$$
m_{d}\left(b_{k}\right)(x) \leq c \gamma \frac{\operatorname{diam}\left(Q_{k}\right)^{n+N_{p}+1}}{\left|x-x_{k}\right|^{n+N_{p}+1}}
$$

if $x \notin Q_{k}^{*}$. Integrating, and using the definition of $N_{p}$, we have that

$$
\int_{D \backslash Q_{k}^{*}} m_{d}\left(b_{k}\right)^{p} d x \leq c \gamma^{p}\left|Q_{k}^{*}\right| \leq c \int_{Q_{k}^{*}} m_{d}(f)^{p} d x .
$$

This proves part 2 of the proposition. We now let $b=\sum b_{k}, g=f-b$. Then $g \in \mathcal{C}_{d}^{\infty}\left(\overline{\mathbf{R}_{+}^{n}}\right)$, so it remains to prove the estimate in part 4 of the proposition. But this is just a straightforward adaptation of the argument on pp. 111-112 of [S2], using the maximal function $m_{d}$ instead of $\mathcal{M}_{0}$, and incorporating the modifications on pp. 104-105 for small values of $p$. Note that in this argument we can ignore the cubes $Q_{k}$ with $\left|Q_{k}^{*}\right| \geq C_{n}$, because for those we have that $g=0$ in $Q_{k}$.

This completes the proof of the proposition.

Proof of Lemma 2.7. Again we will follow the proof in [S2], pp. 107-112. There are a few changes that need to be noted.

First we want to assume that $f$ is locally integrable. This is justified by the fact that $h_{d}^{p}(\bar{D}) \cap L^{1}(D)$ is dense in $h_{d}^{p}(\bar{D})$. To see this it suffices to know that for every $\gamma>0$, the distribution $g$ in the proposition is in fact in $L^{1}(D)$, since letting $\gamma \rightarrow \infty$ we have that $\|f-g\|_{h_{d}^{p}}=\|b\|_{h_{d}^{p}} \rightarrow 0$. Using part 4 of the proposition, we can show (see p. 112 of [S2]) that $m_{d}(g) \in L^{1}\left(\mathbf{R}_{+}^{n}\right)$. Now for every $x \in \mathbf{R}_{+}^{n}$,

$$
m_{d}(g)(x) \geq \sup \langle g, \varphi\rangle
$$

where this time the supremum is taken over all smooth normalized bump functions $\varphi$ with compact support inside $D$. But then $g \in L^{1}$.

Second, convergence is now in the " $h_{d}^{p}(\bar{D})$-norm", i.e. controlled by $\left\|m_{d}(\cdot)\right\|_{L^{p}(D)}^{p}$. As discussed in the remarks following Definition 1.3, this implies convergence in $\mathcal{C}_{d}^{\infty \prime}(\bar{D})$. 
Third, when defining the polynomials $c_{k, l}$, one needs to distinguish between the different kinds of cubes. If any of the cubes involved have volume $\geq C_{n}$, we set the corresponding projection to be zero. Otherwise, if $Q_{l}^{j+1 *}$ is a type (a) cube and $\left|Q_{l}^{j+1 *}\right|<C_{n}$, the projection $P_{l}^{j+1}$ remains as it is in the proof in [S2], i.e. the projection onto the whole space of polynomials $\mathcal{H}_{l, N_{p}}$. However, if $Q_{l}^{j+1 *}$ is a type (b) cube (with $\left|Q_{l}^{j+1 *}\right|<C_{n}$ ), we need to replace $P_{l}^{j+1}$ by $\widetilde{P_{l}^{j+1}}$, the projection onto the subspace of $\mathcal{H}_{l, N_{p}}$ consisting of polynomials divisible by $x_{n}$.

This guarantees that if $Q_{k}^{j *}$ and all of the cubes $Q_{l}^{j+1 *}$ intersecting $Q_{k}^{j}$ are type (a) cubes of volume $<C_{n}$, the atom $A_{k}^{j}$ will have the full moment conditions. On the other hand, if all the cubes are of volume $<C_{n}$ but either $Q_{k}^{j}$ or any of the $Q_{l}^{j+1}$ intersecting it are type (b) cubes, the atom $A_{k}^{j}$ will only have the partial moment conditions corresponding to monomials $x^{\alpha}$ with $\alpha_{n} \geq 1$. It remains to show that in this case $A_{k}^{j}$ will be supported in a type (b) cube.

As in the proof in [S2], we know that $A_{k}^{j}$ is supported in a fixed dilate $c Q_{k}^{j}$ of the cube $Q_{k}^{j *}$. (Actually, the proof in [S2] uses a ball $B_{k}^{j}$, but a cube will do just as well.) Certainly if $Q_{k}^{j *}$ is a type (b) cube, so is $c Q_{k}^{j}$ (here $c \geq 1$, of course). Similarly, if one of the $Q_{l}^{j+1 *}$ is a type (b) cube, i.e. $2 Q_{l}^{j+1 *} \cap \partial \mathbf{R}_{+}^{n} \neq \emptyset$, then since $Q_{l}^{j+1 *} \subset c Q_{k}^{j}$ implies $2 Q_{l}^{j+1 *} \subset 2 c Q_{k}^{j}$, so is $c Q_{k}^{j}$.

Finally, if $Q_{k}^{j *}$ or one of the cubes $Q_{l}^{j+1}$ intersecting it have volume $\geq C_{n}$, then certainly $\left|c Q_{k}^{j}\right| \geq C_{n}$, and so $A_{k}^{j}$ need not have any moment conditions.

The rest of the details are the same as in [S2], and this concludes the proof of the lemma.

\section{The $h_{d}^{p}$ Regularity of the Dirichlet PRoblem}

We now want to study regularity for the Dirichlet problem in the context of the spaces $h_{d}^{p}(\bar{\Omega})$. We begin by looking at the problem in the sense of distributions in $\mathcal{C}_{d}^{\infty \prime}(\bar{\Omega})$.

Let $\Omega \subset \mathbf{R}^{n}$ be a bounded domain with smooth boundary, as above, and let $\mathbf{G}$ be the Green's operator for the Dirichlet problem on $\Omega$, i.e. when $\varphi \in \mathcal{C}^{\infty}(\bar{\Omega})$, $u=\mathbf{G}(\varphi)$ is the solution of the Dirichlet problem

$$
\begin{gathered}
\Delta u=\varphi \text { on } \Omega, \\
u=0 \text { on } \partial \Omega .
\end{gathered}
$$

By the classical theory, we know that for $\varphi \in \mathcal{C}^{\infty}(\bar{\Omega})$ we have $\mathbf{G}(\varphi) \in \mathcal{C}^{\infty}(\bar{\Omega})$, hence $\mathbf{G}(\varphi) \in \mathcal{C}_{d}^{\infty}(\bar{\Omega})$. Moreover, we can consider, for $1 \leq j, l \leq n$, the functions $\frac{\partial^{2} \varphi}{\partial x_{j} \partial x_{l}}$. These are also in $\mathcal{C}^{\infty}(\bar{\Omega})$, and hence $\mathbf{G}\left(\frac{\partial^{2} \varphi}{\partial x_{j} \partial x_{l}}\right)$ will be in $\mathcal{C}_{d}^{\infty}(\bar{\Omega})$. Furthermore, by the Sobolev estimates on $\mathbf{G}$, we know that this map

$$
\mathbf{G} \circ \frac{\partial^{2}}{\partial x_{j} \partial x_{l}}: \mathcal{C}_{d}^{\infty}(\bar{\Omega}) \rightarrow \mathcal{C}_{d}^{\infty}(\bar{\Omega})
$$

is continuous.

This allows us to define a continuous operator on $\mathcal{C}_{d}^{\infty}(\bar{\Omega})$, as follows:

Definition 3.1. For $1 \leq j, l \leq n$, define $T_{j, l}: \mathcal{C}_{d}^{\infty \prime}(\bar{\Omega}) \rightarrow \mathcal{C}_{d}^{\infty \prime}(\bar{\Omega})$ by

$$
\left\langle T_{j, l}(f), \varphi\right\rangle=\left\langle f, \mathbf{G}\left(\frac{\partial^{2} \varphi}{\partial x_{j} \partial x_{l}}\right)\right\rangle
$$

for all $f \in \mathcal{C}_{d}^{\infty}(\bar{\Omega}), \varphi \in \mathcal{C}_{d}^{\infty}(\bar{\Omega})$. 
When $f$ is a smooth function, $T_{j, l}(f)$ takes on a familiar form:

Lemma 3.2. For $f \in \mathcal{C}^{\infty}(\bar{\Omega})$,

$$
T_{j, l}(f)=\frac{\partial^{2} \mathbf{G}(f)}{\partial x_{j} \partial x_{l}}
$$

in $\mathcal{C}_{d}^{\infty}(\bar{\Omega})$.

Proof. Let $f \in \mathcal{C}^{\infty}(\bar{\Omega})$. Then $\frac{\partial^{2} \mathbf{G}(f)}{\partial x_{j} \partial x_{l}} \in \mathcal{C}^{\infty}(\bar{\Omega})$ and for $\varphi \in \mathcal{C}_{d}^{\infty}(\bar{\Omega})$,

$$
\left\langle\frac{\partial^{2} \mathbf{G}(f)}{\partial x_{j} \partial x_{l}}, \varphi\right\rangle=\int_{\Omega} \frac{\partial^{2} \mathbf{G}(f)}{\partial x_{j} \partial x_{l}} \varphi d V
$$

Since $\left.\varphi\right|_{\partial \Omega}=0$, we can integrate by parts without boundary terms to get

$$
-\int_{\Omega} \frac{\partial \mathbf{G}(f)}{\partial x_{l}} \frac{\partial \varphi}{\partial x_{j}} d V
$$

But now since $\left.\mathbf{G}(f)\right|_{\partial \Omega}=0$, we can integrate by parts again, and we have

$$
\int_{\Omega} \mathbf{G}(f) \frac{\partial^{2} \varphi}{\partial x_{j} \partial x_{l}} d V
$$

The Green's operator for the Dirichlet problem is symmetric, so this is the same as

$$
\int_{\Omega} f \mathbf{G}\left(\frac{\partial^{2} \varphi}{\partial x_{j} \partial x_{l}}\right) d V
$$

which is by definition

$$
\left\langle T_{j, l}(f), \varphi\right\rangle \text {. }
$$

Thus we can consider $T_{j, l}$ as an extension to $\mathcal{C}_{d}^{\infty}(\bar{\Omega})$ of the operator $\frac{\partial^{2} \mathbf{G}}{\partial x_{j} \partial x_{l}}$, initially defined on $\mathcal{C}^{\infty}(\bar{\Omega})$. In particular, since $L^{2}(\Omega) \subset \mathcal{C}_{d}^{\infty}(\bar{\Omega})$, this operator is defined on $L^{2}$, and we have the following:

Lemma 3.3. For $1 \leq j, l \leq n, T_{j, l}$ is a bounded operator from $L^{2}(\Omega)$ to $L^{2}(\Omega)$.

This follows by the argument above since the mapping $\psi \rightarrow \partial_{j} \partial_{\ell} \mathbf{G}(\psi)$ extends to a bounded operator on $L^{2}(\Omega)$.

We now come to the main result of this section.

Theorem 3.4. With $\Omega$ as above, and $1 \leq j, l \leq n$, the extension $T_{j, l}$ of $\frac{\partial^{2} \mathbf{G}}{\partial x_{j} \partial x_{l}}$ to $\mathcal{C}_{d}^{\infty \prime}(\bar{\Omega})$ is a bounded operator from $h_{d}^{p}(\bar{\Omega})$ to $h_{d}^{p}(\bar{\Omega})$.

Proof. Let $f \in h_{d}^{p}(\bar{\Omega})$. By the atomic decomposition, we can write

$$
f=\sum \lambda_{k} a_{k}
$$

in $\mathcal{C}_{d}^{\infty}(\bar{\Omega})$, where the $a_{k}$ are either type (a) $h_{d}^{p}$ atoms or type (b) $h_{d}^{p}$ atoms (with respect to some constants $A_{\Omega}, B_{\Omega}$ and $C_{\Omega}$ as in Definition 2.3 and Theorem 2.6 of Section 2.) By the continuity of $T_{j, l}$,

$$
T_{j, l}(f)=\sum \lambda_{k} T_{j, l}\left(a_{k}\right)
$$


in $\mathcal{C}_{d}^{\infty \prime}(\bar{\Omega})$. If we can show that, for every $k, T_{j, l}\left(a_{k}\right) \in h_{d}^{p}(\bar{\Omega})$ and $\left\|T_{j, l}\left(a_{k}\right)\right\|_{h_{d}^{p}} \leq C$ independently of $k$, then we will have that $\sum \lambda_{k} T_{j, l}\left(a_{k}\right)$ converges in $h_{d}^{p}(\bar{\Omega})$ and

$$
\left\|T_{j, l}(f)\right\|_{h_{d}^{p}} \leq C\left(\sum\left|\lambda_{k}\right|^{p}\right)^{1 / p} .
$$

Since this would be true for any atomic decomposition, we will have

$$
\left\|T_{j, l}(f)\right\|_{h_{d}^{p}} \leq C\|f\|_{h_{d}^{p}} .
$$

Therefore, it suffices to prove the following:

Lemma 3.5. If $a$ is an $h_{d}^{p}$ atom (of either type), then $T_{j, l}(a) \in h_{d}^{p}(\bar{\Omega})$ and

$$
\left\|T_{j, l}(a)\right\|_{h_{d}^{p}} \leq C
$$

with $C$ independent of $a$.

Proof of Lemma. Let $g=T_{j, l}(a)$. We want to show $m_{d}(g) \in L^{p}(\Omega)$. For $x \in \Omega$, write

$$
m_{d}(g)(x)=\sup \left\langle g, \varphi_{t}^{x}\right\rangle,
$$

where the supremum is taken over all $\mathcal{C}_{d}^{\infty}$ bump functions $\varphi_{t}^{x}$ supported in balls of radii $t \leq 1$ containing $x$. Let $Q$ be the supporting cube of $a$.

Case I: $x \in \Omega \cap 2 Q$ or $|Q| \geq C_{\Omega}$. Here we will use the $L^{2}$ estimate from Lemma 3.3, namely $g \in L^{2}$ and $\|g\|_{L^{2}} \leq C\|a\|_{L^{2}}$. By extending each $\mathcal{C}_{d}^{\infty}$ bump function to a bump function in $\mathbf{R}^{n}$, we see that

$$
m_{d}(g)(x)=\sup _{\varphi_{t}^{x} \in \mathcal{C}_{d}^{\infty}}\left\langle g, \varphi_{t}^{x}\right\rangle \leq \sup _{\psi_{t}^{x} \in \mathcal{D}\left(\mathbf{R}^{n}\right)}\left\langle g, \psi_{t}^{x}\right\rangle=m(g)(x),
$$

where $m$ is the local grand maximal function in $\mathbf{R}^{n}$. Since $m$ is bounded on $L^{2}$, we have

$$
\left\|m_{d}(g)\right\|_{L^{2}} \leq\|m(g)\|_{L^{2}} \leq C\|g\|_{L^{2}} \leq C^{\prime}\|a\|_{L^{2}} \leq C^{\prime}|Q|^{1 / 2-1 / p},
$$

so

$$
\begin{aligned}
\int_{2 Q \cap \Omega} m_{d}(g)^{p}(x) d x & \leq\left\|m_{d}(g)\right\|_{L^{2}}^{p}|2 Q|^{1-p / 2} \\
& \leq C_{p}\left(|Q|^{1 / 2-1 / p}\right)^{p}|2 Q|^{1-p / 2} \\
& =C_{p}^{\prime} .
\end{aligned}
$$

When $|Q| \geq C_{\Omega}$, we can use the estimate above to bound the integral of $m_{d}(g)^{p}$ over all of $\Omega$, which proves the lemma for the case where $|Q| \geq C_{\Omega}$.

Case II: $|Q|<C_{\Omega}$ and $x \in \Omega \backslash 2 Q$. Fix a $\mathcal{C}_{d}^{\infty}$ bump function $\varphi_{t}^{x}$ with $t \leq 1$. By definition of $T_{j, l}$, we have

$$
\begin{aligned}
\left\langle g, \varphi_{t}^{x}\right\rangle & =\left\langle a, \mathbf{G}\left(\partial_{j} \partial_{l} \varphi_{t}^{x}\right)\right\rangle \\
& =\int_{\Omega} a(y) \mathbf{G}\left(\partial_{j} \partial_{l} \varphi_{t}^{x}\right)(y) d y .
\end{aligned}
$$

Let

$$
K_{\varphi_{t}^{x}}(y)=\mathbf{G}\left(\partial_{j} \partial_{l} \varphi_{t}^{x}\right)(y)
$$


Claim 3.6. The function $K_{\varphi_{t}^{x}}$ is smooth on $\bar{\Omega}$, vanishes identically on the boundary (i.e. $K_{\varphi_{t}^{x}}(y)=0$ for $y \in \partial \Omega$ ), and satisfies the estimates

$$
\left|\frac{\partial^{|\alpha|}}{\partial y^{\alpha}} K_{\varphi_{t}^{x}}(y)\right| \leq \frac{C_{|\alpha|}}{|x-y|^{n+|\alpha|}}
$$

for all multi-indices $\alpha,|\alpha| \leq N_{p}+1$. Here the constants $C_{|\alpha|}$ are independent of the choice of bump function $\varphi_{t}^{x}$.

Assuming the conclusions of the claim, let us proceed with the proof of the lemma. Again there are two cases

Case II(a): $a$ is a type (a) atom with $|Q|<C_{\Omega}$. In this case $Q \subset \Omega$ and we may assume 0 is the center of $Q, Q=[-\delta, \delta]^{n}$ for some $\delta<C_{\Omega}{ }^{1 / n}$.

By Remark 2 following Definition 2.1, and the bounds on the derivatives of $K_{\varphi_{t}^{x}}$ (given by the claim), we have

$$
\begin{aligned}
\left|\int_{Q} a(y) K_{\varphi_{t}^{x}}(y) d y\right| & \leq C_{n, p}\left\|K_{\varphi_{t}^{x}}\right\|_{\mathcal{C}^{N_{p}+1}(Q)}|Q|^{\nu_{p}} \\
& =C_{n, p} \sum_{|\alpha| \leq N_{p}+1} \frac{1}{|\alpha| !} \sup _{y \in Q}\left|\partial_{y}^{\alpha} K_{\varphi_{t}^{x}}(y)\right||Q|^{\nu_{p}} \\
& \leq \sum_{|\alpha| \leq N_{p}+1} \sup _{y \in Q} \frac{C_{|\alpha|}^{\prime}}{|x-y|^{n+|\alpha|}|Q|^{\nu_{p}}} \\
& \leq \sum_{|\alpha| \leq N_{p}+1} \frac{C_{|\alpha|}^{\prime \prime}|Q|^{n+|\alpha|}}{|Q|^{\nu_{p}}} \\
& \leq C \frac{|Q|^{\nu_{p}}}{|x|^{n+N_{p}+1}} .
\end{aligned}
$$

For the next-to-last inequality we used the fact that when $x \notin 2 Q,|x-y| \geq C|x|$ for all $y \in Q$, while for the last inequality we used the fact that $|x|$ is bounded.

Since this bound does not depend on the choice of bump function $\varphi_{t}^{x}$, we get, for $x \in \Omega \backslash 2 Q$,

$$
m_{d}(g)(x)=\sup _{\varphi_{t}^{x}}\left|\int_{Q} a(y) K_{\varphi_{t}^{x}}(y) d y\right| \leq C \frac{|Q|^{\nu_{p}}}{|x|^{n+N_{p}+1}} .
$$

Taking $p$-th powers and integrating over $\Omega \backslash 2 Q$, we see that

$$
\begin{aligned}
|Q|^{p \nu_{p}} \int_{x \in \Omega \backslash 2 Q}|x|^{-\left(n+N_{p}+1\right) p} d x & \leq C \delta^{\left(N_{p}+1\right) p-n+n p} \int_{2 \delta \leq|x| \leq A}|x|^{-\left(n+N_{p}+1\right) p} d x \\
& \leq C
\end{aligned}
$$

since $\left(N_{p}+1\right) p-n+n p=\left(n+N_{p}+1\right) p-n>[n+n(1 / p-1)] p-n=0$. This shows

$$
\int_{x \in \Omega \backslash 2 Q} m_{d}(g)(x)^{p} d x \leq C,
$$

which proves the lemma for the case of a type (a) atom with $|Q|<C_{\Omega}$.

Case II(b): $a$ is a type (b) atom with $|Q|<C_{\Omega}$. Note that by the claim, $K_{\varphi_{t}^{x}} \in$ $\overline{\mathcal{C}_{d}^{\infty}}(\bar{\Omega})$, so by the moment conditions on $a$ (see Definition 2.3 ),

$$
\left|\int_{Q} a(y) K_{\varphi_{t}^{x}}(y) d y\right| \leq\left\|K_{\varphi_{t}^{x}}\right\|_{\mathcal{C}^{N_{p}+1}(Q)}|Q|^{\nu_{p}}
$$


and we can proceed exactly as in case II(a) above to show that

$$
\int_{x \in \Omega \backslash 2 Q} m_{d}(g)(x)^{p} d x \leq C .
$$

This proves the lemma for a type (b) atom with $|Q|<C_{\Omega}$.

Now that we have completed the proof of the lemma, we can get back to the

Proof of Claim 3.6. The smoothness of $K_{\varphi_{t}^{x}}$ on $\bar{\Omega}$ and its vanishing on the boundary follow from the fact that it is the solution to the Dirichlet problem with smooth data $\partial_{j} \partial_{l} \varphi_{t}^{x}$.

To prove the estimates on the derivatives of $K_{\varphi_{t}^{x}}(y)$, we will first consider the situation when $|x-y|<4 t$. Let

$$
u(y)=K_{\varphi_{t}^{x}}(y)=\mathbf{G}\left(\partial_{j} \partial_{l} \varphi_{t}^{x}\right)(y) .
$$

Then it suffices to show

$$
\left|\partial^{\alpha} u\right| \leq C t^{-n-|\alpha|}
$$

for all multi-indices $\alpha$. We will do this by using the Sobolev embedding theorem.

First note that

$$
\left\|\partial^{\alpha} u\right\|_{L^{2}(\Omega)} \leq C t^{-n / 2-|\alpha|} .
$$

For $\alpha=0$, this follows from:

$$
\|u\|_{L^{2}(\Omega)}=\left\|\mathbf{G}\left(\partial_{j} \partial_{l} \varphi_{t}^{x}\right)\right\|_{L^{2}(\Omega)} \leq C\left\|\varphi_{t}^{x}\right\|_{L^{2}(\Omega)} \leq C t^{-n / 2},
$$

since $\varphi_{t}^{x}$ is a bump function. For $|\alpha| \geq 2$, this follows from the regularity of $\mathbf{G}$ :

$$
\left\|\partial^{\alpha} u\right\|_{L^{2}(\Omega)} \leq\|u\|_{|\alpha|} \leq C\left\|\partial_{j} \partial_{l} \varphi_{t}^{x}\right\|_{|\alpha|-2} \leq C t^{-n / 2-|\alpha|} .
$$

Here $\|\cdot\|_{s}$ denotes the Sobolev $s$ norm. Finally, for $|\alpha|=1$, we have, since $u$ satisfies the Dirichlet boundary conditions,

$$
\begin{aligned}
\|\nabla u\|_{L^{2}(\Omega)}^{2} & =\int_{\Omega} \nabla u \cdot \overline{\nabla u} d V \\
& =-\int_{\Omega}(\Delta u) \bar{u} d V \\
& =-\int_{\Omega} \partial_{j} \partial_{l} \varphi_{t}^{x} \bar{u} d V \\
& \leq\left\|\partial_{j} \partial_{l} \varphi_{t}^{x}\right\|_{L^{2}}\|u\|_{L^{2}} \\
& \leq C t^{-n / 2-2} t^{-n / 2} \\
& =C t^{-n-2} .
\end{aligned}
$$

Now fix a multi-index $\alpha$ with $|\alpha| \leq N_{p}+1$. We want to use the Sobolev embedding theorem to bound the $L^{\infty}$ norm of $\partial^{\alpha} u$ by the $L^{2}$ norms of $u$ and its derivatives in balls of radius $t$. Near the boundary, however, such balls may not be contained in $\bar{\Omega}$. Therefore we must first cover a tubular neighborhood of $\partial \Omega$, of radius 1 , by a collection of open sets $U_{i}$, such that each $U_{i}$ is contained in a larger open set $V_{i}$, and in each $V_{i}$ there is a diffeomorphism $\Phi_{i}$ which takes $V_{i} \cap \Omega$ onto the upper half unit ball $B_{+}(0,2)$ and $U_{i} \cap \Omega$ onto $B_{+}(0,1)$. For each $i$, we can extend $u \circ \Phi_{i}^{-1}$ to a smooth function on the whole ball $B(0,2)$ while maintaining its Sobolev norms (see [S1], Chapter VI, Section 3). These are essentially the Sobolev norms of $u$, up to a constant depending on $\Phi_{i}$. 
Fixing $y \in \bar{\Omega}$, since $t \leq 1$, we have either $B(y, t) \subset \bar{\Omega}$, or $y \in U_{i}$ for some $i$. In the latter case, by making a change of variables, we may assume $y \in B_{+}(0,1)$, hence $B(y, t) \subset B(0,2)$. Replacing $u$ by $u \circ \Phi_{i}^{-1}$, and extending to $B(0,2)$, we may assume that $u$ is defined in $B(y, t)$ and its Sobolev norms are bounded as above.

For simplicity, make a translation so that $y=0$. Thus we have

$$
\left\|\partial^{\beta} u\right\|_{L^{2}(B(0, t))} \leq C t^{-n / 2-|\beta|} .
$$

Now define $v$ in $B(0,1)$ by $v(z)=u(t z)$. Then it is easy to see that

$$
\left\|\partial^{\beta} v\right\|_{L^{2}(B(0,1))}=\left\|\partial^{\beta} u\right\|_{L^{2}(B(0, t))} t^{|\beta|-n / 2} \leq C t^{-n} .
$$

Let $k$ be an integer greater than $n / 2$. By the Sobolev embedding theorem, applied to the function $\partial^{\alpha} v$, we can write

$$
\sup _{B(0,1 / 2)}\left\|\partial^{\alpha} v\right\| \leq C \sum_{|\gamma| \leq k}\left\|\partial^{\gamma}\left(\partial^{\alpha} v\right)\right\|_{L^{2}(B(0,1))} \leq C t^{-n}
$$

(see [S1], pp. 124-130). But $\partial^{\alpha} v(z)=t^{|\alpha|}\left(\partial^{\alpha} u\right)(t z)$ so

$$
\left|\partial^{\alpha} u(0)\right| \leq t^{-|\alpha|} \sup _{B(0,1 / 2)}\left\|\partial^{\alpha} v\right\| \leq C t^{-n-|\alpha|} .
$$

Recalling that $\partial^{\alpha} u(0)=\frac{\partial^{|\alpha|}}{\partial y^{\alpha}} K_{\varphi_{t}^{x}}(y)$, we see that we are done with the case $|x-y| \leq$ $4 t$.

When $|x-y|>4 t$, we write

$$
K_{\varphi_{t}^{x}}(y)=\int_{\Omega} G(y, z) \frac{\partial^{2} \varphi_{t}^{x}}{\partial z_{j} \partial z_{l}}(z) d z
$$

Here $y$ is outside the support of $\varphi_{t}^{x}$, so the integral is taken only over the region where $G(y, z)$ is smooth in $z$. In addition, both $\varphi_{t}^{x}$ and $G$ vanish on the boundary, so we can integrate by parts to get

$$
K_{\varphi_{t}^{x}}(y)=\int_{\Omega} \partial_{z_{j}} \partial_{z_{l}} G(y, z) \varphi_{t}^{x}(z) d z .
$$

Differentiating in $y$, this gives

$$
\frac{\partial^{|\alpha|}}{\partial y^{\alpha}} K_{\varphi_{t}^{x}}(y)=\int_{\Omega} \partial_{y}^{\alpha} \partial_{z_{j}} \partial_{z_{l}} G(y, z) \varphi_{t}^{x}(z) .
$$

Furthermore, for $z$ in the support of $\varphi_{t}^{x}$ we have

$$
|x-y| \leq|x-z|+|z-y| \leq 2 t+|z-y|<|x-y| / 2+|z-y| ;
$$

hence $|x-y| / 2<|y-z|$. Thus if we can show

$$
\left|\partial_{y}^{\alpha} \partial_{z_{j}} \partial_{z_{l}} G(y, z)\right| \leq C|y-z|^{-n-|\alpha|}
$$

for all $z \in \Omega \backslash\{y\}$, we would get

$$
\begin{aligned}
\left|\frac{\partial^{|\alpha|}}{\partial y^{\alpha}} K_{\varphi_{t}^{x}}(y)\right| & \leq\left|\int_{\Omega} \partial_{y}^{\alpha} \partial_{z_{j}} \partial_{z_{l}} G(y, z) \varphi_{t}^{x}(z) d z\right| \\
& \leq \int_{\Omega} \frac{C}{|y-z|^{n+|\alpha|}}\left|\varphi_{t}^{x}(z)\right| d z \\
& \leq C|x-y|^{-n-|\alpha|}
\end{aligned}
$$

which is the desired estimate. 
Proof of estimate (3.1). We will follow the general outline of the proofs in [CKS], Section 5 , but without using the reflection mapping. We start by writing the Green's operator $\mathbf{G}$, acting on some $f \in \mathcal{C}^{\infty}(\bar{\Omega})$, as

$$
\mathbf{G}(f)=\mathbf{E}(f)+\mathbf{H}(f) .
$$

Here $\mathbf{E}(f)=E * f$ is convolution with the Newtonian potential $E$, while $-\mathbf{H}(f)=$ $\mathbf{P}\left(\left.E * f\right|_{\partial \Omega}\right)$ is the Poisson integral of the restriction of $\mathbf{E}(f)$ to the boundary. Thus we can write the Green's function as

$$
G(y, z)=E(y, z)+H(y, z)
$$

where

$$
E(y, z)=c_{n}|y-z|^{-(n-2)}
$$

and $H(y, z)$ is the kernel of the operator $\mathbf{H}$. Now $E$ is symmetric in $y$ and $z$ and its second derivatives form a Calderón-Zygmund kernel, so the inequality (3.1) holds with $E$ instead of $G$. Thus we need only concentrate on $H$.

In order to estimate the derivatives of $H(y, z)$, we write, for $z \in \Omega \backslash\{y\}$,

$$
\begin{aligned}
\frac{\partial^{2}}{\partial z_{j} \partial z_{l}} H(y, z) & =\frac{\partial^{2}}{\partial z_{j} \partial z_{l}} \int_{\partial \Omega} P(y, \zeta) E(\zeta, z) d \sigma(\zeta) \\
& =\int_{\partial \Omega} P(y, \zeta) \frac{\partial^{2}}{\partial z_{j} \partial z_{l}} E(\zeta, z) d \sigma(\zeta),
\end{aligned}
$$

where $P(y, \zeta)$ is the Poisson kernel in $\Omega$, and $d \sigma$ is surface measure on $\partial \Omega$. We differentiate the integral with respect to $y$ and separate into local and global parts by means of a cut-off function $\phi:(0, \infty) \rightarrow \mathbf{R}$ such that $\phi(t)=1$ for $t<1 / 4$ and $\phi(t)=0$ for $t>1 / 2$ :

$$
\begin{aligned}
\frac{\partial^{|\alpha|}}{\partial y^{\alpha}} & \int_{\partial \Omega} P(y, \zeta) \frac{\partial^{2}}{\partial z_{j} \partial z_{l}} E(\zeta, z) d \sigma(\zeta) \\
= & \int_{\partial \Omega} \partial_{y}^{\alpha} P(y, \zeta) \phi\left(\frac{|\zeta-z|}{|y-z|}\right) \partial_{z_{j}} \partial_{z_{l}} E(\zeta, z) d \sigma(\zeta) \\
& \quad+\int_{\partial \Omega} \partial_{y}^{\alpha} P(y, \zeta)\left[1-\phi\left(\frac{|\zeta-z|}{|y-z|}\right)\right] \partial_{z_{j}} \partial_{z_{l}} E(\zeta, z) d \sigma(\zeta) \\
= & I_{1}+I_{2} .
\end{aligned}
$$

We want to estimate the local part, $I_{1}$ (where $|\zeta-z| \leq|y-z| / 2$ ), by means of integration by parts. In order to do this, we first have to convert the $z$ derivatives of $E$ into $\zeta$ derivatives. Note that because of the symmetric form of $E$, we have

$$
\partial_{z_{j}} \partial_{z_{l}} E(\zeta, z)=\partial_{\zeta_{j}} \partial_{\zeta_{l}} E(\zeta, z) .
$$

Next, for $z, y$ fixed and $\zeta$ in a neighborhood of $\partial \Omega$, with $|\zeta-z| \leq|y-z| / 2$, we choose tangential and normal coordinates $\left(\zeta^{\prime}, \rho\right)$ in $\zeta$. Converting the old derivatives to the new ones, we get

$$
\frac{\partial}{\partial \zeta_{j}}=\sum_{k=1}^{n-1} a_{j, k} \frac{\partial}{\partial \zeta_{k}^{\prime}}+b_{j} \frac{\partial}{\partial \rho}
$$

for some smooth functions $a_{j, k}, b_{j}$, and therefore

$$
\frac{\partial^{2}}{\partial \zeta_{j} \partial \zeta_{l}} E(\zeta, z)=\sum_{|\beta|+k \leq 2} c_{\beta, k} \frac{\partial^{|\beta|+k}}{\partial\left(\zeta^{\prime}\right)^{\beta} \partial \rho^{k}} E(\zeta, z) .
$$


By choosing $\rho$ to be the signed geodesic distance to $\partial \Omega$, we can rewrite the equation $\Delta_{\zeta} E(\zeta, z)=0$ in the new coordinates as

$$
\frac{\partial^{2}}{\partial \rho^{2}} E(\zeta, z)=\sum_{1 \leq i, m \leq n-1} d_{i, m} \frac{\partial^{2}}{\partial \zeta_{i}^{\prime} \partial \zeta_{m}^{\prime}} E(\zeta, z)+\text { first order terms. }
$$

This reduces the number of $\rho$ derivatives by one, and we remain with an expression of the form

$$
\sum_{|\beta|+k \leq 2, k=0,1} g_{\beta, k}(\zeta) \frac{\partial^{|\beta|+k}}{\partial\left(\zeta^{\prime}\right)^{\beta} \partial \rho^{k}} E(\zeta, z)
$$

for the second derivatives $\partial_{j} \partial_{l}$ of $E(\zeta, z)$. Inserting this into $I_{1}$, we get

$$
\sum_{|\beta|+k \leq 2, k=0,1} \int_{\partial \Omega} \partial_{y}^{\alpha} P(y, \zeta) \phi\left(\frac{|\zeta-z|}{|y-z|}\right) g_{\beta, k}(\zeta) \frac{\partial^{|\beta|+k}}{\partial\left(\zeta^{\prime}\right)^{\beta} \partial \rho^{k}} E(\zeta, z) d \sigma(\zeta) .
$$

We are now ready to integrate by parts in the tangential variables $\zeta^{\prime}$. The derivatives can fall either on $\partial_{y}^{\alpha} P(\eta, \zeta)$, on $\phi\left(\frac{|\zeta-z|}{|y-z|}\right)$, or on $g_{\beta, k}(\zeta)$, and we use the following estimates:

$$
\begin{aligned}
\left|\partial_{\zeta^{\prime}}^{\gamma} \partial_{y}^{\alpha} P(y, \zeta)\right| & \leq C|\zeta-y|^{-(n-1+|\gamma|+|\alpha|)} \\
& \leq C|y-z|^{-(n-1+|\gamma|+|\alpha|)}
\end{aligned}
$$

since $|\zeta-y| \geq|y-z|-|\zeta-z| \geq|y-z| / 2$,

$$
\left|\partial_{\zeta^{\prime}}^{\gamma} \phi\left(\frac{|\zeta-z|}{|y-z|}\right)\right| \leq C|y-z|^{-|\gamma|}
$$

and

$$
\left|\partial_{\zeta^{\prime}}^{\gamma} g_{\beta, k}(\zeta)\right| \leq C
$$

Combining, and using the fact that we can always choose the largest negative exponent of $|y-z|$, since it is bounded above, we have

$$
\begin{aligned}
\left|I_{1}\right| \leq & \frac{C_{1}}{|y-z|^{n+1+|\alpha|}} \int_{|\zeta-z| \leq|y-z| / 2}|E(\zeta, z)| d \sigma(\zeta) \\
& +\frac{C_{2}}{|y-z|^{n+|\alpha|}} \int_{|\zeta-z| \leq|y-z| / 2}\left|\frac{\partial}{\partial \rho} E(\zeta, z)\right| d \sigma(\zeta) \\
\leq & \frac{C}{|y-z|^{n+1+|\alpha|}} \int_{|\zeta-z| \leq|y-z| / 2}|\zeta-z|^{-(n-2)} d \sigma(\zeta) \\
& +\frac{C}{|y-z|^{n+|\alpha|}} \int_{\partial \Omega}\left|\partial_{\nu_{\zeta}} E(\zeta, z)\right| d \sigma(\zeta) \\
\leq & C|y-z|^{-(n+|\alpha|)} .
\end{aligned}
$$

The last estimate (bounding the integral of the normal derivative of the Newtonian potential by a constant) can be found in $[\mathrm{F}]$ (Lemma 3.20, p. 165).

The global part of the integral, $I_{2}$, can be estimated in the same way as was done in $[\mathrm{CKS}]$, in the proof of Lemma 5.3 (pp. 327-331), i.e. by using integration by parts to transfer derivatives from the Poisson kernel to the Newtonian potential. Here we must use the estimate

$$
\begin{aligned}
\left|\partial_{\zeta^{\prime}}^{\gamma} E(\zeta, z)\right| & \leq C|\zeta-z|^{-(n-2+|\gamma|)} \\
& \leq C|y-z|^{-(n-2+|\gamma|)}
\end{aligned}
$$


for $|\zeta-z| \geq|y-z| / 4$. Since the largest $|\gamma|$ possible is $|\alpha|+2$, we have

$$
\left|I_{2}\right| \leq C|y-z|^{-(n+|\alpha|)},
$$

and so all in all

$$
\left|\frac{\partial^{|\alpha|}}{\partial y^{\alpha}} H(y, z)\right|=\left|\frac{\partial^{|\alpha|}}{\partial y^{\alpha}} \int_{\partial \Omega} P(y, \zeta) \frac{\partial^{2}}{\partial z_{j} \partial z_{l}} E(\zeta, z) d \sigma(\zeta)\right| \leq C|y-z|^{-(n+|\alpha|)} .
$$

Combined with the equivalent estimates on $E(y, z)$, we get estimate (3.1).

Now the proof of Claim 3.6 is complete.

This also concludes the proof of Theorem 3.4, the $h_{d}^{p}$ regularity of the Dirichlet problem.

\section{The $h_{z}^{p}$ Regularity of the Dirichlet and Neumann problems}

In this section we will study the regularity of the Dirichlet and Neumann problems in the context of the spaces $h_{z}^{p}(\bar{\Omega})$. We shall concentrate on the Neumann problem, since it involves the more difficult analysis.

Let $\Omega \subset \mathbf{R}^{n}$ be a bounded domain with smooth boundary, and let $\widetilde{\mathbf{G}}$ be a solution operator for the Neumann problem, defined on $f \in \mathcal{C}^{\infty}(\bar{\Omega})$ with $\int_{\Omega} f=0$ by $\widetilde{\mathbf{G}}(f)=u$, where

$$
\begin{gathered}
\Delta u=f \text { on } \Omega, \\
\frac{\partial u}{\partial \vec{n}}=0 \text { on } \partial \Omega,
\end{gathered}
$$

and $\int_{\Omega} u=0$. Here $\vec{n}$ is the outward unit normal vector field on $\partial \Omega$.

If $f \in \mathcal{C}^{\infty}(\bar{\Omega})$ with $\int_{\Omega} f=0$, then $\widetilde{\mathbf{G}}(f) \in \mathcal{C}^{\infty}(\bar{\Omega})$, so we can apply the operators $\frac{\partial^{2} \widetilde{\mathbf{G}}}{\partial x_{j} \partial x_{l}}, j, l=1, \ldots, n$, to $f$. We want to extend these operators to $h_{z}^{p}(\bar{\Omega})$. To do this, we will proceed through the $L^{2}$ theory.

As is well known, $\widetilde{\mathbf{G}}$ extends to a bounded operator from $L^{2}(\Omega)$ to the Sobolev space $H_{2}(\Omega)$, so for $j, l=1, \ldots, n$, the operators $\frac{\partial^{2} \widetilde{\mathbf{G}}}{\partial x_{j} \partial x_{l}}$, extend to bounded operators on $L^{2}(\Omega)$. Thus we can define $\frac{\partial^{2} \widetilde{\mathbf{G}}}{\partial x_{j} \partial x_{l}}(a)$ for an $h_{z}^{p}$ atom $a$. We shall prove the following

Theorem 4.1. With $\Omega$ as above, and $1 \leq j, l \leq n$, there is an extension $\widetilde{T}_{j, l}$ of $\frac{\partial^{2} \widetilde{\mathbf{G}}}{\partial x_{j} \partial x_{l}}$ to a bounded operator from $h_{z}^{p}(\bar{\Omega})$ to $h_{z}^{p}(\bar{\Omega})$.

Before proceeding with the proof of this theorem, we shall state the corresponding result for the Dirichlet problem. As above, one can also define the operator $\frac{\partial^{2} \mathbf{G}}{\partial x_{j} \partial x_{l}}$ on $h_{z}^{p}$ atoms, where $\mathbf{G}$ is the solution operator of the Dirichlet problem for the Laplacian, as in Section 3. Thus we have

Theorem 4.2. With $\Omega$ as above, and $1 \leq j, l \leq n$, there is an extension of $\frac{\partial^{2} \mathbf{G}}{\partial x_{j} \partial x_{l}}$ to a bounded operator from $h_{z}^{p}(\bar{\Omega})$ to $h_{z}^{p}(\bar{\Omega})$.

The proof of this result is just a minor modification of the proof of Theorem 4.1, so we will omit it. In Section 5 , we will give a different proof for the case $p=1$.

The essence of the proof of Theorem 4.1 is contained in the following 
Lemma 4.3. If $a$ is an $h_{z}^{p}$ atom, then $\frac{\partial^{2} \widetilde{\mathbf{G}}}{\partial x_{j} \partial x_{l}}(a) \in h_{z}^{p}(\bar{\Omega})$ and

$$
\left\|\frac{\partial^{2} \widetilde{\mathbf{G}}}{\partial x_{j} \partial x_{l}}(a)\right\|_{h_{z}^{p}} \leq C,
$$

with $C$ independent of $a$.

Proof of lemma. Let $g=\frac{\partial^{2} \widetilde{\mathbf{G}}}{\partial x_{j} \partial x_{l}}(a)$. Note that by definition, we are taking $g=0$ on $\mathbf{R}^{n} \backslash \bar{\Omega}$. Thus to show $g \in h_{z}^{p}(\bar{\Omega})$, we only have to show $g \in h^{p}\left(\mathbf{R}^{n}\right)$. Let $m$ be the local grand maximal function, as in Definition 1.1. Then we want to show $m(g) \in L^{p}\left(\mathbf{R}^{n}\right)$. In fact, we only need to show $m(g) \in L^{p}(\widetilde{\Omega})$, where $\widetilde{\Omega}$ is some bounded set containing all points of distance at most 2 from $\bar{\Omega}$.

Let $Q$ be the supporting cube of $a$.

Case I: $x \in 2 Q$ or $|Q|>1$. Here we will use an $L^{2}$ estimate. Since both $\frac{\partial^{2} \widetilde{\mathbf{G}}}{\partial x_{j} \partial x_{l}}$ and $m$ are bounded on $L^{2}$, we have

$$
\|m(g)\|_{L^{2}} \leq C\|g\|_{L^{2}} \leq C\|a\|_{L^{2}} \leq C|Q|^{1 / 2-1 / p} .
$$

Thus

$$
\begin{aligned}
\int_{2 Q} m(g)^{p}(x) d x & \leq\|m(g)\|_{L^{2}}^{p}|2 Q|^{1-p / 2} \\
& \leq C\left(|Q|^{1 / 2-1 / p}\right)^{p}|2 Q|^{1-p / 2} \\
& =C .
\end{aligned}
$$

When $|Q|>1$, we can use the estimate above to bound the integral of $m(g)^{p}$ over all of $\widetilde{\Omega}$, thus proving the lemma for that case.

Case II: $|Q|<1$ and $x \notin 2 Q$. Fix a $\mathcal{C}^{\infty}$ bump function $\varphi_{t}^{x}$ with $t \leq 1$. Then we have

$$
\begin{aligned}
\left\langle g, \varphi_{t}^{x}\right\rangle & =\int_{\Omega} \frac{\partial^{2} \widetilde{\mathbf{G}}}{\partial x_{j} \partial x_{l}}(a)(x) \varphi_{t}^{x}(x) d x \\
& =\int_{\Omega}\left\{\int_{\Omega} \frac{\partial^{2}}{\partial x_{j} \partial x_{l}} \widetilde{G}(x, y) a(y) d y\right\} \varphi_{t}^{x}(x) d x \\
& =\int_{\Omega}\left\{\int_{\Omega} \frac{\partial^{2}}{\partial x_{j} \partial x_{l}} \widetilde{G}(x, y) \varphi_{t}^{x}(x) d x\right\} a(y) d y \\
& =\int_{\Omega} \widetilde{K_{\varphi_{t}^{x}}}(y) a(y) d y,
\end{aligned}
$$

where

$$
\widetilde{K_{\varphi_{t}^{x}}}(y)=\int_{\Omega} \frac{\partial^{2}}{\partial z_{j} \partial z_{l}} \widetilde{G}(z, y) \varphi_{t}^{x}(z) d z
$$

i.e. the dual operator $\left(\partial_{j} \partial_{l} \widetilde{\mathbf{G}}\right)^{*}$ applied to $\varphi_{t}^{x}$.

Claim 4.4. The function $\widetilde{K_{\varphi_{t}^{x}}}$ is smooth on $\bar{\Omega}$ and satisfies the estimates

$$
\left|\frac{\partial^{|\alpha|}}{\partial y^{\alpha}} \widetilde{K_{\varphi_{t}^{x}}}(y)\right| \leq \frac{C_{|\alpha|}}{|x-y|^{n+|\alpha|}}
$$

for all multi-indices $\alpha,|\alpha| \leq N_{p}+1$. Here the constants $C_{|\alpha|}$ are independent of the choice of bump function $\varphi_{t}^{x}$. 
Assuming the conclusions of the claim, let us continue with the proof of Case II of the lemma. Recall that $Q \subset \bar{\Omega}$. We may assume 0 is the center of $Q$, and $Q=[-\delta, \delta]^{n}$ for some $\delta<1$. Using the moment conditions on $a$ and the bounds on the derivatives of $\widetilde{K_{\varphi_{t}^{x}}}$ given by the claim, we can proceed exactly as in Case II(a) of the proof of Lemma 3.5 to get that

$$
\begin{aligned}
m(g)(x) & =\sup _{\varphi_{t}^{x}}\left|\int_{Q} a(y) \widetilde{K_{\varphi_{t}^{x}}}(y) d y\right| \\
& \leq \frac{C|Q|^{\nu_{p}}}{|x|^{n+N_{p}+1}} .
\end{aligned}
$$

and

$$
\begin{aligned}
\int_{x \in \tilde{\Omega} \backslash 2 Q} m(g)(x)^{p} d x & \leq C \delta^{p n \nu_{p}} \int_{2 \delta \leq|x| \leq \tilde{A}}|x|^{-\left(n+N_{p}+1\right) p} d x \\
& \leq C .
\end{aligned}
$$

This proves Case II of the lemma, and the proof of the lemma is complete.

Proof of Claim 4.4. We will first prove that the operator $\left(\partial_{j} \partial_{l} \widetilde{\mathbf{G}}\right)^{*}$ is bounded from the Sobolev space $H_{k}(\Omega)$ to itself, $k=0,1,2, \ldots$ Specifically, we want to show that if $\varphi \in \mathcal{C}^{\infty}(\bar{\Omega})$, then $\left(\partial_{j} \partial_{l} \widetilde{\mathbf{G}}\right)^{*}(\varphi) \in H_{k}(\Omega)$ for all $k$, with

$$
\left\|\left(\partial_{j} \partial_{l} \widetilde{\mathbf{G}}\right)^{*}(\varphi)\right\|_{k} \leq C\|\varphi\|_{k}
$$

where $\|\cdot\|_{k}$ denotes the Sobolev $k$ norm on $\Omega$. Since $\varphi_{t}^{x} \in \mathcal{C}^{\infty}(\bar{\Omega})$, this will show that $\widetilde{K_{\varphi_{t}^{x}}}=\left(\partial_{j} \partial_{l} \widetilde{\mathbf{G}}\right)^{*}\left(\varphi_{t}^{x}\right)$ is smooth up to the boundary.

To prove 4.1, we first take a smooth partition of unity $\left\{\eta_{\mu}\right\}, \mu=0, \ldots, M$, where as usual $\eta_{0}$ has compact support in $\Omega$, and for $\mu \geq 1, \eta_{\mu}$ has compact support in an open set $U_{\mu}$ which comes equipped with a system of tangential and normal coordinates $\left(\zeta^{\prime}, \rho\right), \rho$ being the geodesic distance to $\partial \Omega$. Write

$$
\left(\partial_{j} \partial_{l} \widetilde{\mathbf{G}}\right)^{*}(\varphi)=\sum_{\mu=0}^{M}\left(\partial_{j} \partial_{l} \widetilde{\mathbf{G}}\right)^{*}\left(\eta_{\mu} \varphi\right) .
$$

We want to prove 4.1 for each $\eta_{\mu} \varphi$.

When $\mu=0$, since $\eta_{0} \varphi$ has compact support in $\Omega$, we can integrate by parts, and since $\widetilde{\mathbf{G}}$ is self-dual, we have that

$$
\left(\partial_{j} \partial_{l} \widetilde{\mathbf{G}}\right)^{*}\left(\eta_{0} \varphi\right)=\widetilde{\mathbf{G}}\left(\partial_{j} \partial_{l}\left(\eta_{0} \varphi\right)\right)
$$

Thus we can use the classical Sobolev estimates for the Neumann problem to get (4.1) for $\eta_{0} \varphi_{t}^{x}$. See Section 3, proof of Claim 3.6 for the analogous argument in the case of the Dirichlet problem.

Now fix $\mu \geq 1$, and let $U=U_{\mu}, \eta=\eta_{\mu}$. Making the change of variables $\Phi(z)=\left(\zeta^{\prime}, \rho\right)=\zeta$, and letting $\tau=\left[(\eta \varphi) \circ \Phi^{-1}\right] J_{\Phi}^{-1}$, we have

$$
\begin{aligned}
\left(\partial_{j} \partial_{l} \widetilde{\mathbf{G}}\right)^{*}(\eta \varphi)(y)= & \int_{U \cap \Omega} \frac{\partial^{2}}{\partial z_{j} \partial z_{l}} \widetilde{G}(z, y) \eta(z) \varphi(z) d z \\
= & \sum_{1 \leq i, m \leq n} \int_{\mathbf{R}_{+}^{n}} a_{i, m}^{j, l}(\zeta) \frac{\partial^{2}}{\partial \zeta_{i} \partial \zeta_{m}} \widetilde{G}\left(\Phi^{-1}(\zeta), y\right) \tau(\zeta) d \zeta \\
& +\sum_{1 \leq m \leq n} \int_{\mathbf{R}_{+}^{n}} b_{m}^{j, l}(\zeta) \frac{\partial}{\partial \zeta_{m}} \widetilde{G}\left(\Phi^{-1}(\zeta), y\right) \tau(\zeta) d \zeta
\end{aligned}
$$


We will deal with each term in the sums individually, and distinguish between tangential and normal derivatives.

Case I: Tangential derivatives. In this case, we want to integrate by parts to transfer the derivatives from $\widetilde{G}$ to $\varphi$, and use the Sobolev estimates for $\widetilde{G}$. Because of the change of coordinates, there are certain details to keep track of.

Consider the function $f \in L^{2}(\Omega)$ defined by

$$
f(y)=\int_{\mathbf{R}_{+}^{n}} a(\zeta) \frac{\partial^{2}}{\partial \zeta_{i} \partial \zeta_{m}} \widetilde{G}\left(\Phi^{-1}(\zeta), y\right) \tau(\zeta) d \zeta,
$$

with $i, m \leq n-1$, where $a=a_{i, m}^{j, l}$ is smooth. Testing $f$ against some $\psi \in \mathcal{C}^{\infty}(\bar{\Omega})$, we can integrate by parts to get

$$
\begin{aligned}
\int_{\Omega} f(y) \psi(y) d y & =\int_{\Omega}\left\{\int_{\mathbf{R}_{+}^{n}} a(\zeta) \frac{\partial^{2}}{\partial \zeta_{i} \partial \zeta_{m}} \widetilde{G}\left(y, \Phi^{-1}(\zeta)\right) \tau(\zeta) d \zeta\right\} \psi(y) d y \\
& =\int_{\mathbf{R}_{+}^{n}}\left\{\int_{\Omega} \frac{\partial^{2}}{\partial \zeta_{i} \partial \zeta_{m}} \widetilde{G}\left(\Phi^{-1}(\zeta), y\right) \psi(y) d y\right\} a(\zeta) \tau(\zeta) d \zeta \\
& =\int_{\mathbf{R}_{+}^{n}} \frac{\partial^{2}}{\partial \zeta_{i} \partial \zeta_{m}} \widetilde{\mathbf{G}}(\psi)\left(\Phi^{-1}(\zeta)\right) a(\zeta) \tau(\zeta) d \zeta \\
& =\int_{\mathbf{R}_{+}^{n}} \widetilde{\mathbf{G}}(\psi)\left(\Phi^{-1}(\zeta)\right) \frac{\partial^{2}}{\partial \zeta_{i} \partial \zeta_{m}}[a(\zeta) \tau(\zeta)] d \zeta \\
& =\int_{\Omega} \widetilde{\mathbf{G}}(\psi)(z) \phi(z) J_{\Phi}(z) d z \\
& =\int_{\Omega} \psi \widetilde{\mathbf{G}}\left(\phi J_{\Phi}\right),
\end{aligned}
$$

where

$$
\phi=\frac{\partial^{2}}{\partial \zeta_{i} \partial \zeta_{m}}(a \tau) \circ \Phi .
$$

This shows $f=\widetilde{\mathbf{G}}\left(\phi J_{\Phi}\right)$ in $L^{2}(\Omega)$, so the Sobolev estimates for $\widetilde{G}$, combined with the fact that

$$
\|\phi\|_{k} \leq C_{\Phi}\|\varphi\|_{k+2}
$$

for all $k \geq 0$, give the desired estimate 4.1 for the terms in the sum 4.3 with $i, m \leq n-1$. A similar argument gives an even better estimate for the terms in the sum 4.4 with $m \leq n-1$.

Case II: One normal derivative. This is the case of a term in the sum 4.3 or 4.4 with $m=n$. If in addition we have a tangential derivative, we may integrate by parts as above. Thus what remains is to show that the operator $\widetilde{\mathbf{G}}_{\rho}^{*}$, defined on $\varphi \in \mathcal{C}^{\infty}(\bar{\Omega})$ by

$$
\widetilde{\mathbf{G}}_{\rho}^{*}(\varphi)(y)=\int_{\Omega} \frac{\partial}{\partial \rho_{z}} \widetilde{G}(y, z) \varphi(z) d z,
$$

is bounded from $H_{k}(\Omega)$ to $H_{k+1}(\Omega)$. Recall (see [CKS], Section 7) that we can write the solution operator $\widetilde{\mathbf{G}}$ to the Neumann problem as

$$
\widetilde{\mathbf{G}}=\mathbf{E}+\widetilde{\mathbf{H}}+S,
$$


where $\mathbf{E}$ is convolution with the Newtonian potential $E$ (followed by restriction to $\bar{\Omega})$

$$
\widetilde{\mathbf{H}}=-\mathbf{P}\left(Q \mathbf{R} \frac{\partial}{\partial \rho} \mathbf{E}\right),
$$

and $S$ is a smoothing error. Here $Q$ is smoothing of order 1 on the boundary.

Since $\mathbf{E}$ maps $H_{k}(\Omega)$ to $H_{k+2}(\Omega)$, its derivatives are bounded from $H_{k}(\Omega)$ to $H_{k+1}(\Omega)$. Furthermore, denoting the kernel by $E(y, z)$, we have

$$
\frac{\partial}{\partial \rho_{z}} E(y, z)=\sum c_{m}(z) \frac{\partial}{\partial z_{m}} E(y, z)=-\sum c_{m}(z) \frac{\partial}{\partial y_{m}} E(y, z),
$$

for some smooth functions $c_{m}$. Thus the operator $\mathbf{E}_{\rho}^{*}$ defined by

$$
\mathbf{E}_{\rho}^{*}(\varphi)(y)=\int_{\Omega} \frac{\partial}{\partial \rho_{z}} E(y, z) \varphi(z) d z
$$

also maps $H_{k}(\Omega)$ to $H_{k+1}(\Omega)$.

Now let us look at $\widetilde{\mathbf{H}}_{\rho}^{*}$, again defined by

$$
\widetilde{\mathbf{H}}_{\rho}^{*}(\varphi)(y)=\int_{\Omega} \frac{\partial}{\partial \rho_{z}} \widetilde{H}(y, z) \varphi(z) d z,
$$

where $\widetilde{H}$ is the kernel of $\widetilde{\mathbf{H}}$. Looking at 4.5 , we see that since we are differentiating with respect to the variable of integration, the derivative falls on the innermost operator, namely $\mathbf{E}$, so that

$$
\widetilde{\mathbf{H}}_{\rho}^{*}=-\mathbf{P}\left(Q \mathbf{R} \frac{\partial}{\partial \rho} \mathbf{E}_{\rho}^{*}\right)
$$

Using 4.6, we see that

$$
\frac{\partial}{\partial \rho_{z}} E(y, z)+\frac{\partial}{\partial \rho_{y}} E(y, z)=\sum\left[c_{m}(y)-c_{m}(z)\right] \frac{\partial}{\partial y_{m}} E(y, z),
$$

which shows that the operator

$$
\mathbf{E}_{\rho}^{*}-\frac{\partial}{\partial \rho} \mathbf{E}
$$

is smoothing of order 2 on $\bar{\Omega}$. Thus

$$
\widetilde{\mathbf{H}}_{\rho}^{*}=\mathbf{P}\left(Q \mathbf{R} \frac{\partial^{2}}{\partial \rho^{2}} \mathbf{E}\right)+\widetilde{\mathbf{H}}^{\prime}
$$

where $\widetilde{\mathbf{H}}^{\prime}$ has the same regularity properties as $\widetilde{\mathbf{H}}$, namely it maps $H_{k}(\Omega)$ to $H_{k+2}(\Omega)$.

But now note that since $E$ is harmonic, we can write $\frac{\partial^{2}}{\partial \rho^{2}} \mathbf{E}$ in terms of purely tangential derivatives, and at most one normal derivative. The tangential derivatives commute with the operators $\mathbf{R}, Q$ and $\mathbf{P}$ up to lower order terms, so that in the end we remain with operators of the form $\partial_{j} \partial_{l} \widetilde{\mathbf{H}}$, which we know have the desired regularity (see $[\mathrm{CKS}]$ ), plus operators which are smoothing to at least order 1. As for the remaining normal derivative, it gives an operator which is essentially $\widetilde{\mathbf{H}}$, up to multiplication by a smooth function. Thus we see that we can write $\widetilde{\mathbf{H}}_{\rho}^{*}$ as a sum of operators, all of which map $H_{k}(\Omega)$ to $H_{k+1}(\Omega)$.

Finally, looking at the smoothing operator $S$, since its kernel can be taken to be as smooth as we like, applying $\frac{\partial}{\partial \rho_{z}}$ to it still gives a kernel which is smoothing. 
Thus we have shown that $\widetilde{\mathbf{G}}_{\rho}^{*}$ maps $H_{k}(\Omega)$ to $H_{k+1}(\bar{\Omega})$, which proves the Sobolev estimates 4.1 for any term containing one normal derivative.

Case III: Two normal derivatives. Here we look at the operator with kernel $\frac{\partial^{2}}{\partial \rho_{z}^{2}} \widetilde{G}(y, z)$. We use the fact that the Neumann function $\widetilde{G}(y, z)$ is harmonic in both $y$ and $z$, so again we can write this in terms of purely tangential derivatives and at most one normal derivative, which cases were dealt with above.

Putting everything together, we see that the estimates (4.1) are satisfied for each of the terms in the sums 4.3 and 4.4; hence they are satisfied for $\eta_{\mu} \varphi$ for each $\mu$, and we have shown that the operator $\left(\partial_{j} \partial_{l} \widetilde{\mathbf{G}}\right)^{*}$ is bounded from $H_{k}(\Omega)$ to $H_{k}(\Omega)$, and in particular $\widetilde{K_{\varphi_{t}^{x}}}$ is smooth up to the boundary.

To prove the estimates on the derivatives of $\widetilde{K_{\varphi_{t}^{x}}}(y)$, we will first consider the situation when $|x-y|<4 t$. Then it suffices to show

$$
\left|\partial^{\alpha} \widetilde{K_{\varphi_{t}^{x}}}\right| \leq C t^{-n-|\alpha|}
$$

for all multi-indices $\alpha$. This can be done by using the Sobolev embedding theorem, as in the case of the Dirichlet problem (see Section 3, proof of Claim 3.6). We only need the estimates

$$
\left\|\partial^{\alpha} \widetilde{K_{\varphi_{t}^{x}}}\right\|_{L^{2}(\Omega)} \leq C t^{-n / 2-|\alpha|},
$$

which follow from the regularity of $\left(\frac{\partial^{2} \widetilde{\mathbf{G}}}{\partial x_{j} \partial x_{l}}\right)^{*}$ discussed above:

$$
\left\|\partial^{\alpha} \widetilde{K_{\varphi_{t}^{x}}}\right\|_{L^{2}(\Omega)} \leq\left\|\widetilde{K_{\varphi_{t}^{x}}}\right\|_{|\alpha|} \leq C\left\|\varphi_{t}^{x}\right\|_{|\alpha|} \leq C t^{-n / 2-|\alpha|} .
$$

When $|x-y|>4 t$, we write

$$
\widetilde{K_{\varphi_{t}^{x}}}(y)=\int_{\Omega} \frac{\partial^{2}}{\partial z_{j} \partial z_{l}} \widetilde{G}(y, z) \varphi_{t}^{x}(z) d z .
$$

Here $y$ is outside the support of $\varphi_{t}^{x}$, so the integral is taken only over the region where $\widetilde{G}(y, z)$ is smooth in $z$. Differentiating in $y$, this gives

$$
\frac{\partial^{|\alpha|}}{\partial y^{\alpha}} \widetilde{K_{\varphi_{t}^{x}}}(y)=\int_{\Omega} \partial_{y}^{\alpha} \partial_{z_{j}} \partial_{z_{l}} \widetilde{G}(y, z) \varphi_{t}^{x}(z) d z
$$

Furthermore, for $z$ in the support of $\varphi_{t}^{x}$ we have

$$
|x-y| \leq|x-z|+|z-y| \leq 2 t+|z-y|<|x-y| / 2+|z-y|
$$

hence $|x-y| / 2<|y-z|$. Thus if we can show

$$
\left|\partial_{y}^{\alpha} \partial_{z_{j}} \partial_{z_{l}} \widetilde{G}(y, z)\right| \leq C|y-z|^{-n-|\alpha|}
$$

for all $z \in \Omega \backslash\{y\}$, we would get

$$
\begin{aligned}
\left|\frac{\partial^{|\alpha|}}{\partial y^{\alpha}} \widetilde{K_{\varphi_{t}^{x}}}(y)\right| & \leq\left|\int_{\Omega} \partial_{y}^{\alpha} \partial_{z_{j}} \partial_{z_{l}} \widetilde{G}(y, z) \varphi_{t}^{x}(z) d z\right| \\
& \leq \int_{\Omega} \frac{C}{|y-z|^{n+|\alpha|}}\left|\varphi_{t}^{x}(z)\right| d z \\
& \leq C|x-y|^{-n-|\alpha|}
\end{aligned}
$$

which is the desired estimate.

So it remains to prove the estimate (4.7) for the Neumann function $\widetilde{G}$. Again we write

$$
\widetilde{\mathbf{G}}=\mathbf{E}+\widetilde{\mathbf{H}}+S .
$$


The Newtonian potential $E(y, z)$ is symmetric in $y$ and $z$ and its second derivatives form a Calderón-Zygmund kernel, so the inequality (4.7) holds with $E$ instead of $\widetilde{G}$. Furthermore, the kernel for $S$ can be taken to be as smooth as desired. Thus we need only concentrate on the kernel $\widetilde{H}(y, z)$ of $\widetilde{\mathbf{H}}$.

In order to estimate the derivatives of $\widetilde{H}(y, z)$, again write

$$
\widetilde{\mathbf{H}}=-\mathbf{P}\left(Q \mathbf{R} \frac{\partial}{\partial \rho} \mathbf{E}\right) .
$$

Let $T=Q \mathbf{R} \frac{\partial}{\partial \rho} \mathbf{E}$. Since $Q$ is smoothing of order 1 on $\partial \Omega, T$ is a reverse Poisson operator of the same order as RE. When we consider $\partial_{y}^{\alpha} \partial_{z_{j}} \partial_{z_{l}} \widetilde{H}(y, z)$, the $y$ derivatives fall on the Poisson kernel $P$, while the $z$ derivatives fall on the kernel of $T$. If $K(\zeta, z)$ is this kernel $(\zeta \in \partial \Omega, z \in \bar{\Omega})$, then

$$
\partial_{y}^{\alpha} \partial_{z_{j}} \partial_{z_{l}} \widetilde{H}(y, z)=\int_{\partial \Omega} \partial_{y}^{\alpha} P(y, \zeta) \partial_{z_{j}} \partial_{z_{l}} K(\zeta, z)
$$

We can now proceed as in the proof of Claim 3.6 (for the Dirichlet problem), with $K(\zeta, z)$ instead of the kernel $E(\zeta, z)$ of RE. Namely, we use integration by parts to transfer derivatives between $K$ and $P$. In the "local" case (denoted by $I_{1}$ in the proof of Claim 3.6), we can use the harmonicity of $E$ and integration by parts to express $\partial_{z_{j}} \partial_{z_{l}} K(\zeta, z)$ as

$$
\sum_{|\beta| \leq 2} \partial_{\zeta}^{|\beta|} K_{\beta}(\zeta, z)
$$

where the derivatives in $\zeta$ are tangential, and the operators associated to the kernels $K_{\beta}$ are of the same (or lower) order as $T$. Thus we can integrate by parts to move the derivatives onto $P$. For the "global" case $\left(I_{2}\right)$, we transfer the derivatives from $P$ onto $K$.

Alternatively, one can proceed to prove the estimates on the derivatives of the kernel $\widetilde{H}(y, z)$ via the symbolic calculus, as in [CKS] (see pp. 342-346, although in this case one needs to reverse the variables when taking derivatives).

This gives the estimate (4.7) on the derivatives of $\widetilde{G}$, which in turn proves Claim 4.4.

Now we can finally give the

Proof of Theorem 4.1. Let $f \in h_{z}^{p}(\bar{\Omega})$. By the atomic decomposition, we can write

$$
f=\sum \lambda_{k} a_{k}
$$

in $\mathcal{S}^{\prime}\left(\mathbf{R}^{n}\right)$, where the $a_{k}$ are $h_{z}^{p}$ atoms. By Lemma 4.3, for every $k, \frac{\partial^{2} \widetilde{\mathbf{G}}}{\partial x_{j} \partial x_{l}}\left(a_{k}\right) \in$ $h_{z}^{p}(\bar{\Omega})$, and $\left\|\frac{\partial^{2} \widetilde{\mathbf{G}}}{\partial x_{j} \partial x_{l}}\left(a_{k}\right)\right\|_{h_{z}^{p}} \leq C$ independently of $a_{k}$, so that $\sum \lambda_{k} \frac{\partial^{2} \widetilde{\mathbf{G}}}{\partial x_{j} \partial x_{l}}\left(a_{k}\right)$ converges in the $h_{z}^{p}(\bar{\Omega})$ norm. Since $h_{z}^{p}(\bar{\Omega})$ is a closed subspace of $h_{z}^{p}\left(\mathbf{R}^{n}\right)$, hence complete, this sum is an element of $h_{z}^{p}(\bar{\Omega})$, and we can define $\widetilde{T}_{j, l}$ on $f$ by

$$
\widetilde{T}_{j, l}(f) \stackrel{\text { def }}{=} \sum \lambda_{k} \frac{\partial^{2} \widetilde{\mathbf{G}}}{\partial x_{j} \partial x_{l}}\left(a_{k}\right) .
$$

To see that this is independent of the choice of atomic decomposition, it suffices to show that $\widetilde{T}_{j, l}$ is continuous on $L^{2}(\Omega)$ in the distribution topology, i.e. if $\left\{f_{k}\right\}$ is 
a sequence in $L^{2}(\Omega)$ with $f_{k} \rightarrow 0$ in $\mathcal{C}^{\infty}(\bar{\Omega})$, then

$$
\left\langle\frac{\partial^{2} \widetilde{\mathbf{G}}}{\partial x_{j} \partial x_{l}}\left(f_{k}\right), \varphi\right\rangle \rightarrow 0
$$

for every $\varphi \in \mathcal{C}^{\infty}(\bar{\Omega})$. But as in Case II of the proof of Lemma 4.3,

$$
\left\langle\frac{\partial^{2} \widetilde{\mathbf{G}}}{\partial x_{j} \partial x_{l}}\left(f_{k}\right), \varphi\right\rangle=\left\langle f_{k},\left(\partial_{j} \partial_{l} \widetilde{\mathbf{G}}\right)^{*}(\varphi)\right\rangle .
$$

The right-hand-side converges to zero since $f_{k} \rightarrow 0$ in $\mathcal{C}^{\infty}(\bar{\Omega})$ and the operator $\left(\partial_{j} \partial_{l} \widetilde{\mathbf{G}}\right)^{*}$ is continuous on $\mathcal{C}^{\infty}(\bar{\Omega})$, as demonstrated in the proof of Claim 4.4 .

Thus the extension $\widetilde{T}_{j, l}$ of $\frac{\partial^{2} \widetilde{\mathbf{G}}}{\partial x_{j} \partial x_{l}}$ to $h_{z}^{p}(\bar{\Omega})$ is well defined. Furthermore, we have that

$$
\left\|\widetilde{T}_{j, l}(f)\right\|_{h_{z}^{p}} \leq C\left(\sum\left|\lambda_{k}\right|^{p}\right)^{1 / p}
$$

with $C$ independent of the atomic decomposition. Since

$$
\|f\|_{h_{z}^{p}(\bar{\Omega})} \approx \inf \left(\sum\left|\lambda_{k}\right|^{p}\right)^{1 / p}
$$

where the infimum is taken over all such decompositions, we get

$$
\left\|\widetilde{T}_{j, l}(f)\right\|_{h_{z}^{p}} \leq C^{\prime}\|f\|_{h_{z}^{p}}
$$

i.e. $\widetilde{T}_{j, l}$ is bounded on $h_{z}^{p}(\bar{\Omega})$.

We have now proved the $h_{z}^{p}$ regularity of the Neumann problem.

\section{5. $H^{1}$ AND BMO REGULARITY}

In this section we will give a different proof of the $h_{z}^{1}$ regularity of the Dirichlet and Neumann problems for the Laplacian. Then we will then state and prove the corresponding results for the appropriate dual space, $\mathrm{bmo}_{r}$, and for the dual space of $h_{d}^{1}(\bar{\Omega}), \mathrm{bmo}_{z}$.

Let us state again the case $p=1$ of Theorems 4.1 and 4.2.

Theorem 5.1. If $\Omega$ is as above, and $\mathbf{G}, \widetilde{\mathbf{G}}$ are the solution operators for the Dirichlet and Neumann problems, respectively, then for $1 \leq j, l \leq n$, the operators $\frac{\partial^{2} \mathbf{G}}{\partial x_{j} \partial x_{l}}$ and $\frac{\partial^{2} \widetilde{\mathbf{G}}}{\partial x_{j} \partial x_{l}}$ extend to bounded operators from $h_{z}^{1}(\bar{\Omega})$ to $h_{z}^{1}(\bar{\Omega})$.

Instead of using the maximal function, we will prove this by using a cancellation property to obtain the atomic decomposition directly. To illustrate this method, we will first prove an analogue in the upper half-space. Just as for the local spaces $h_{z}^{p}(\bar{\Omega})$, we define the space $H_{z}^{p}\left(\overline{\mathbf{R}_{+}^{n}}\right)$ to be the subspace of $H^{p}\left(\mathbf{R}^{n}\right)$ consisting of those distributions supported in $\overline{\mathbf{R}_{+}^{n}}$.

Proposition 5.2. If $\mathbf{G}$ (resp. $\widetilde{\mathbf{G}}$ ) is the solution operator for the Dirichlet problem (resp. Neumann problem) on the upper half-space $\mathbf{R}_{+}^{n}$, then for $1 \leq j, l \leq n, \frac{\partial^{2} \mathbf{G}}{\partial x_{j} \partial x_{l}}$ (resp. $\left.\frac{\partial^{2} \widetilde{\mathbf{G}}}{\partial x_{j} \partial x_{l}}\right)$ extends to a bounded operator from $H_{z}^{1}\left(\overline{\mathbf{R}_{+}^{n}}\right)$ to $H_{z}^{1}\left(\overline{\mathbf{R}_{+}^{n}}\right)$.

Proof. Let $f \in H_{z}^{1}\left(\overline{\mathbf{R}_{+}^{n}}\right)$. Then by an analogue of Theorem 2.5 (see [JSW], Theorem 5.3, and [CKS], Theorem 3.3), $f$ has an atomic decomposition $\sum \lambda_{j} a_{j}$, where $\sum\left|\lambda_{j}\right|<\infty$ and the $a_{j}$ are now $H^{1}$ atoms supported in cubes $Q_{j}$ contained entirely 
in $\overline{\mathbf{R}_{+}^{n}}$. Recall that an $H^{1}$ atom must satisfy a size condition, which in this case we take in the $L^{2}$ sense:

$$
\|a\|_{L^{2}} \leq|Q|^{-1 / 2}
$$

and an exact moment condition:

$$
\int a(x) d x=0 .
$$

As in the proofs of Theorem 3.4 and Theorem 4.1, it suffices to show that the operators are bounded in the $H_{z}^{1}$ norm when acting on atoms.

Consider such an atom $a$. Then $a \in L^{2}$, so we can define the solutions to the Dirichlet and Neumann problems for $a$ by $u=\mathbf{G}(a)=E_{o} * a$ and $u=\widetilde{\mathbf{G}}(a)=E_{e} * a$, respectively. Here $E$ is the Newtonian potential, and $E_{o}, E_{e}$ are its odd and even parts. Let

$$
F_{j, l}=\frac{\partial^{2} u}{\partial x_{j} \partial x_{l}} .
$$

We want to show that for any $1 \leq j, l \leq n,\left.F_{j, l}\right|_{\overline{\mathbf{R}_{+}^{n}}} \in H_{z}^{1}\left(\overline{\mathbf{R}_{+}^{n}}\right)$ with norm bounded by a constant.

We begin by proving a cancellation property for $F_{j, l}$, namely that

$$
\int_{\mathbf{R}_{+}^{n}} F_{j, l}=0 .
$$

A priori, we know $F_{j, l}$ exists locally in the $L^{2}$ sense, and is therefore locally integrable. We will show that it is actually integrable at infinity, so $F_{j, l} \in L^{1}\left(\mathbf{R}_{+}^{n}\right)$. Note that

for the Dirichlet problem, or

$$
F_{j, l}=\frac{\partial^{2} E_{o}}{\partial x_{j} \partial x_{l}} * a
$$

$$
F_{j, l}=\frac{\partial^{2} E_{e}}{\partial x_{j} \partial x_{l}} * a
$$

for the Neumann problem. Let $Q$ be the supporting cube of $a$, with center $y_{Q}$. Then by the moment condition on $a$, and the size of the derivatives of the Newtonian potential, we have, for $x \in \mathbf{R}^{n} \backslash 2 Q$ :

$$
\begin{aligned}
\left|F_{j, l}(x)\right| & \leq\left|\int_{Q} \frac{\partial^{2} E_{o}}{\partial x_{j} \partial x_{l}}(x-y) a(y) d y\right| \\
& =\left|\int_{Q}\left\{\frac{\partial^{2} E_{o}}{\partial x_{j} \partial x_{l}}(x-y)-\frac{\partial^{2} E_{o}}{\partial x_{j} \partial x_{l}}\left(x-y_{Q}\right)\right\} a(y) d y\right| \\
& \leq \frac{C}{\left|x-y_{Q}\right|^{n+1}} \int_{Q}\left|y-y_{Q}\right||a(y)| d y \\
& \leq \frac{C|Q|^{1 / n}}{\left|x-y_{Q}\right|^{n+1}} .
\end{aligned}
$$

Exactly the same estimate holds for the case of the Neumann problem.

Now that we have the decay and integrability at infinity, we immediately get that

$$
\int_{\mathbf{R}_{+}^{n}} \frac{\partial^{2} u}{\partial x_{j} \partial x_{l}} d x=0
$$


whenever either $j<n$ or $l<n$. Furthermore, since $\Delta u=a$ on $\mathbf{R}_{+}^{n}$, and $a$ has vanishing integral, we have

$$
\int_{\mathbf{R}_{+}^{n}} \frac{\partial^{2} u}{\partial x_{n}^{2}} d x=\int_{\mathbf{R}_{+}^{n}}\left\{a-\sum_{i=1}^{n-1} \frac{\partial^{2} u}{\partial x_{i}^{2}}\right\} d x=0 .
$$

Thus $\int_{\mathbf{R}_{+}^{n}} F_{j, l}=0$ for all $j, l$.

Now fix $j$ and $l$ and let $F=F_{j, l}$. We will show $\left.F\right|_{\mathbf{R}_{+}^{n}}$ has an atomic decomposition with atoms supported entirely in $\overline{\mathbf{R}_{+}^{n}}$.

Again looking at the supporting cube $Q$ of $a$, we define $Q_{k}, k \geq 1$, to be the smallest cube such that

$$
\left(2^{k} Q\right) \cap \overline{\mathbf{R}_{+}^{n}} \subset Q_{k} \subset \overline{\mathbf{R}_{+}^{n}} .
$$

Set

for $k \geq 1$,

$$
F_{Q_{k}}=\frac{1}{\left|Q_{k}\right|} \int_{Q_{k}} F(x) d x
$$

$$
g_{1}=F \chi_{Q_{1}}-F_{Q_{1}} \chi_{Q_{1}},
$$

and

$$
g_{k}=F \chi_{Q_{k} \backslash Q_{k-1}}+F_{Q_{k-1}} \chi_{Q_{k-1}}-F_{Q_{k}} \chi_{Q_{k}}
$$

for $k \geq 2$. Then $\int g_{1}=0$ and for $k \geq 2$,

$$
\int g_{k}=\int_{Q_{k} \backslash Q_{k-1}} F+\left\{\frac{1}{\left|Q_{k-1}\right|} \int_{Q_{k-1}} F\right\}\left|Q_{k-1}\right|-\left\{\frac{1}{\left|Q_{k}\right|} \int_{Q_{k}} F\right\}\left|Q_{k}\right|=0 .
$$

We claim

$$
\sum_{i=1}^{k} g_{i}=F \chi_{Q_{k}}-F_{Q_{k}} \chi_{Q_{k}} .
$$

This is true for $k=1$, and if we assume it is true for $k-1$, we get

$$
\begin{aligned}
\sum_{i=1}^{k} g_{i}= & \sum_{i=1}^{k-1} g_{i}+g_{k} \\
= & F \chi_{Q_{k-1}}-F_{Q_{k-1}} \chi_{Q_{k-1}} \\
& +F \chi_{Q_{k} \backslash Q_{k-1}}+F_{Q_{k-1}} \chi_{Q_{k-1}}-F_{Q_{k}} \chi_{Q_{k}} \\
= & F \chi_{Q_{k}}-F_{Q_{k}} \chi_{Q_{k}} .
\end{aligned}
$$

This, combined with the fact that $\int_{\mathbf{R}_{+}^{n}} F=0$, gives

$$
\begin{aligned}
\int_{\mathbf{R}_{+}^{n}}\left|F-\sum_{i=1}^{k} g_{i}\right| & \leq \int_{\mathbf{R}_{+}^{n}}\left|F-F \chi_{Q_{k}}\right|+\left|F_{Q_{k}}\right|\left|Q_{k}\right| \\
& =\int_{\mathbf{R}_{+}^{n} \backslash Q_{k}}|F|+\left|\int_{Q_{k}} F\right| \\
& =\int_{\mathbf{R}_{+}^{n} \backslash Q_{k}}|F|+\left|\int_{\mathbf{R}_{+}^{n} \backslash Q_{k}} F\right| \\
& \leq 2 \int_{\mathbf{R}_{+}^{n} \backslash Q_{k}}|F| \\
& \rightarrow 0
\end{aligned}
$$


as $k \rightarrow \infty$. Thus

$$
\left.F\right|_{\mathbf{R}_{+}^{n}}=\sum_{i=1}^{\infty} g_{i}
$$

in $L^{1}$.

Now we want to estimate the $L^{2}$ norms of the $g_{k}$. Note that for $k=1$, we have, by the $L^{2}$ boundedness,

$$
\begin{aligned}
\left\|g_{1}\right\|_{L^{2}} & \leq\left\{\int_{Q_{1}}|F|^{2}\right\}^{1 / 2}+\left|F_{Q_{1}} \| Q_{1}\right|^{1 / 2} \\
& \leq 2\left\{\int_{Q_{1}}|F|^{2}\right\}^{1 / 2} \\
& \leq 2\left\|\frac{\partial^{2} u}{\partial x_{j} \partial x_{l}}\right\|_{L^{2}} \\
& \leq C\|a\|_{L^{2}} \\
& \leq C_{1}\left|Q_{1}\right|^{-1 / 2}
\end{aligned}
$$

For $k \geq 2$ we can again write

$$
\left\|g_{k}\right\|_{L^{2}} \leq\left\{\int_{Q_{k} \backslash Q_{k-1}}|F|^{2}\right\}^{1 / 2}+\left|F_{Q_{k-1}}\right|\left|Q_{k-1}\right|^{1 / 2}+\left|F_{Q_{k}}\right|\left|Q_{k}\right|^{1 / 2} .
$$

To estimate the first term, recall that, for $x \in \mathbf{R}_{+}^{n} \backslash Q_{1}$,

$$
|F(x)| \leq \frac{C|Q|^{1 / n}}{\left|x-y_{Q}\right|^{n+1}} .
$$

Therefore, with $\delta$ being one-half the sidelength of $Q$, we have

$$
\begin{aligned}
\left\{\int_{Q_{k} \backslash Q_{k-1}}|F|^{2}\right\}^{1 / 2} & \leq \frac{C \delta}{\left(2^{k-1} \delta\right)^{n+1}}\left|Q_{k}\right|^{1 / 2} \\
& =C 2^{-k(n / 2+1)} \delta^{-n / 2} \\
& =C 2^{-k}\left|Q_{k}\right|^{-1 / 2} .
\end{aligned}
$$

As for the second and third terms, we can use the fact that $\int_{\mathbf{R}_{+}^{n}} F=0$, and the estimate for $F(x)$ in $\mathbf{R}_{+}^{n} \backslash Q_{1}$, to get:

$$
\begin{aligned}
\left|F_{Q_{k}}\right| & =\frac{1}{\left|Q_{k}\right|}\left|\int_{Q_{k}} F(x) d x\right| \\
& =\frac{1}{\left|Q_{k}\right|}\left|\int_{\mathbf{R}_{+}^{n} \backslash Q_{k}} F(x) d x\right| \\
& \leq \frac{C|Q|^{1 / n}}{\left|Q_{k}\right|} \int_{\left|x-y_{Q}\right| \geq 2^{k} \delta} \frac{1}{\left|x-y_{Q}\right|^{n+1}} d x \\
& \leq C \frac{\delta}{\left(2^{k} \delta\right)^{n+1}},
\end{aligned}
$$


So

$$
\begin{aligned}
\left|F_{Q_{k}}\right|\left|Q_{k}\right|^{1 / 2} & \leq C \frac{\delta}{\left(2^{k} \delta\right)^{n+1}}\left|Q_{k}\right|^{1 / 2} \\
& =C 2^{-k}\left|Q_{k}\right|^{-1 / 2} .
\end{aligned}
$$

The estimate for $k-1$ is the same up to a constant. Thus we see that

$$
\left\|g_{k}\right\|_{L^{2}} \leq C 2^{-k}\left|Q_{k}\right|^{-1 / 2}
$$

Now let $\lambda_{k}=C 2^{-k}$ for $k \geq 2, \lambda_{1}=C_{1}$, and $a_{k}=\lambda_{k}^{-1} g_{k}$. Then each $a_{k}$ is supported in $Q_{k} \subset \overline{\mathbf{R}_{+}^{n}}$, and satisfies $\int a_{k}=0$ and $\left\|a_{k}\right\|_{L^{2}} \leq\left|Q_{k}\right|^{-1 / 2}$, so it is an $H_{z}^{1}\left(\overline{\mathbf{R}_{+}^{n}}\right)$ atom. Also $F=\sum \lambda_{k} a_{k}$, and

$$
\sum_{k=1}^{\infty}\left|\lambda_{k}\right|=C_{1}+C \sum_{k=2}^{\infty} 2^{-k}=A<\infty
$$

Here $A$ is independent of $a$.

This completes the proof of Proposition 5.2.

Proof of Theorem 5.1. We are going to give a local version of the proof of Proposition 5.2. We will give the proof for the case of the Dirichlet problem, and indicate where changes must be made for the Neumann problem. Again, it suffices to consider an $h_{z}^{1}$ atom $a$. Let $u=\mathbf{G}(a)$, fix $j, l$, and set

$$
F=\frac{\partial^{2} u}{\partial x_{j} \partial x_{l}}
$$

We will give an atomic decomposition for $F$ in terms of modified $h_{z}^{1}$ atoms (see Remark 3 following Definition 2.3).

We must distinguish between the case where $a$ is supported "away from the boundary", and the case where $a$ is supported "near the boundary". Cover $\bar{\Omega}$ by open sets $U_{0}, \ldots, U_{M}$, where $\overline{U_{0}} \subset \Omega, \partial \Omega \subset \bigcup_{i=1}^{M} U_{i}$, and in each $U_{i}, i=1, \ldots, M$, we have a system of tangential and normal coordinates $\left(t_{1}, \ldots, t_{n}\right)$. Let $C_{\Omega}>0$ be such that if $B$ is a ball with $|B|<C_{\Omega}$, then $B \cap \bar{\Omega} \subset U_{i}$ for some $i$. If $Q$ is the supporting cube of $a$, and $|Q|<C_{\Omega}$, let $K$ be the largest integer such that $\left|2^{K} Q\right|<C_{\Omega}$, and set

$$
Q_{K}=2^{K} Q \cap \bar{\Omega}
$$

Then one of the following cases must hold:

Case 0: $|Q| \geq C_{\Omega}$. In this case, we treat $F$ as a multiple of a large modified $h_{z}^{1}$

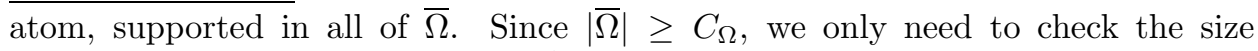
condition, which follows from the $L^{2}$ estimates:

$$
\|F\|_{L^{2}}=\left\|\frac{\partial^{2} u}{\partial x_{j} \partial x_{l}}\right\|_{L^{2}} \leq C\|a\|_{L^{2}} \leq C C_{\Omega}{ }^{-1 / 2} .
$$

This shows that the $h_{z}^{1}$ norm of $F$ is bounded by a constant independent of $a$. Case 1: $|Q|<C_{\Omega}$ and $Q_{K} \subset U_{0}$. By rotating and translating, we can assume $Q=$ $[-\delta, \delta]^{n}$ for some $\delta>0$.

In order to use get the atomic decomposition for $F$, we need an analogue of the cancellation property 5.1. We start by getting the size estimates on $F$ and the first derivatives of $u$. If $G$ is the Green's function for the Dirichlet problem, then

$$
F(x)=\int_{\Omega} \frac{\partial^{2} G(x, y)}{\partial x_{j} \partial x_{l}} a(y) d y .
$$


By interchanging the role of the variables in estimate 3.1 and its proof, we get that

$$
\left|\partial_{y}^{\alpha} \partial_{x_{j}} \partial_{x_{l}} G(x, y)\right| \leq C|x-y|^{-n-|\alpha|}
$$

for $0 \leq|\alpha| \leq 1$. The same holds for the Neumann function $\widetilde{G}$, following the proof of estimate (4.7). Combined with the moment condition on $a$, this gives

$$
\begin{aligned}
|F(x)| & \leq \frac{C}{|x|^{n+1}} \int_{Q}|y||a(y)| d y+\frac{C}{|x|^{n}}\left|\int_{Q} a(y) d y\right| \\
& \leq \frac{C|Q|^{1 / n}}{|x|^{n+1}}+\frac{C|Q|^{\nu_{1}}}{|x|^{n}} \\
& \leq \frac{C^{\prime}|Q|^{1 / n}}{|x|^{n+1}} .
\end{aligned}
$$

Note that we were able to absorb the second term because $\nu_{1}=1 / n$, and $|x|$ is bounded above. Applying the same estimates to the first order derivatives, and combining terms, we also get

$$
\left|\frac{\partial u}{\partial x_{l}}\right| \leq \frac{C|Q|^{1 / n}}{|x|^{n}}
$$

Thus we can integrate $F$ in one variable first and use this estimate to get

$$
\begin{aligned}
\left|\int_{Q_{K}} F(x) d x\right| & =\left|\int_{\left[-2^{K} \delta, 2^{K} \delta\right]^{n-1}}\left\{\int_{-2^{K} \delta}^{2^{K} \delta} \frac{\partial}{\partial x_{j}}\left(\frac{\partial u}{\partial x_{l}}\right) d x_{j}\right\} d x^{\prime}\right| \\
& \leq \frac{2 C|Q|^{1 / n}}{\left|2^{K} \delta\right|^{n}} \operatorname{Vol}_{n-1}\left(\left[-2^{K} \delta, 2^{K} \delta\right]^{n-1}\right) \\
& \leq C|Q|^{1 / n}
\end{aligned}
$$

as $2^{K} \delta \approx C_{\Omega}{ }^{1 / n}$. This is the approximate cancellation condition which replaces equation (5.1).

Now we proceed to define, as in the case of the upper half-space: $Q_{k}=2^{k} Q$ and $F_{Q_{k}}=\frac{1}{\left|Q_{k}\right|} \int_{Q_{k}} F(x) d x$ for $1 \leq k \leq K$,

$$
g_{1}=F \chi_{Q_{1}}-F_{Q_{1}} \chi_{Q_{1}},
$$

and

$$
g_{k}=F \chi_{Q_{k} \backslash Q_{k-1}}+F_{Q_{k-1}} \chi_{Q_{k-1}}-F_{Q_{k}} \chi_{Q_{k}}
$$

for $2 \leq k \leq K$. We also set $Q_{K+1}=\bar{\Omega}$ and

$$
g_{K+1}=F \chi_{\Omega \backslash Q_{K}}+F_{Q_{K}} \chi_{Q_{K}} .
$$

Then again we have that $g_{k}$ is supported in $Q_{k}, \int g_{k}=0$ for $1 \leq k \leq K$, and

$$
\sum_{k=1}^{K+1} g_{k}=F \chi_{Q_{K}}-F_{Q_{K}} \chi_{Q_{K}}+g_{K+1}=\left.F\right|_{\Omega} .
$$

The estimates for the $L^{2}$ norms of the $g_{k}$ have to be modified to take into account the approximate cancellation conditions. While we still have

$$
\left\|g_{1}\right\|_{L^{2}} \leq C\|a\|_{L^{2}} \leq C_{1}\left|Q_{1}\right|^{-1 / 2},
$$


and for $2 \leq k \leq K$,

$$
\left\{\int_{Q_{k} \backslash Q_{k-1}}|F|^{2}\right\}^{1 / 2} \leq C 2^{-k}\left|Q_{k}\right|^{-1 / 2},
$$

we no longer have $\int F=0$, but rather $\left|\int_{Q_{K}} F\right| \leq C|Q|^{1 / n}$. This gives

$$
\begin{aligned}
\left|F_{Q_{k}}\right| & =\frac{1}{\left|Q_{k}\right|}\left|\int_{Q_{k}} F(x) d x\right| \\
& \leq \frac{1}{\left|Q_{k}\right|}\left\{\left|\int_{Q_{K} \backslash Q_{k}} F(x) d x\right|+\left|\int_{Q_{K}} F(x) d x\right|\right\} \\
& \leq \frac{C|Q|^{1 / n}}{\left|Q_{k}\right|}\left\{\int_{2^{k} \delta \leq|x| \leq c}|x|^{-(n+1)} d x+1\right\} \\
& \leq C \frac{\delta}{\left(2^{k} \delta\right)^{(n+1)}} .
\end{aligned}
$$

Note that here we again used the fact that $2^{K} \delta \approx C_{\Omega}{ }^{1 / n}$. Hence

$$
\left|F_{Q_{k}}\right|\left|Q_{k}\right|^{1 / 2} \leq C 2^{-k}\left|Q_{k}\right|^{-1 / 2}
$$

as above. Combining these estimates, we once more get, for $2 \leq k \leq K$,

$$
\left\|g_{k}\right\|_{L}^{2} \leq 2^{-k}\left|Q_{k}\right|^{-1 / 2}
$$

For $K+1$, we have

$$
\begin{aligned}
\left\|g_{K+1}\right\|_{L^{2}} & \leq\left\{\int_{\Omega \backslash Q_{K}}|F|^{2}\right\}^{1 / 2}+\left|F_{Q_{K}} \| Q_{K}\right|^{1 / 2} \\
& \leq C_{\Omega}|Q|^{1 / n} \\
& \leq C_{\Omega} .
\end{aligned}
$$

This shows $g_{K+1} \in h_{z}^{1}(\bar{\Omega})$ with norm bounded by a constant, so we only need to consider $F-g_{K+1}$.

Set $\lambda_{1}=C_{1}$ and $\lambda_{k}=C 2^{-k}$ for $2 \leq k \leq K$. Then $g_{k}=\lambda_{k} a_{k}$ where each $a_{k}$ is supported in $Q_{k} \subset \bar{\Omega}, \int_{Q_{k}} a_{k}=0$ when $\left|Q_{k}\right| \leq\left|Q_{K}\right|<C_{\Omega}$, and $\left\|a_{k}\right\|_{L^{2}} \leq\left|Q_{k}\right|^{-1 / 2}$. Thus each $a_{k}$ is a modified $h_{z}^{1}(\bar{\Omega})$ atom. Also $F-g_{K+1}=\sum \lambda_{k} a_{k}$ in $\Omega$, and

$$
\sum_{k=1}^{K}\left|\lambda_{k}\right| \leq C_{1}+C \sum_{k=2}^{K} 2^{-k} \leq C .
$$

Case 2: $|Q|<C_{\Omega}$ and $Q_{K} \subset U_{i}$ for some $i=1, \ldots, n$. From the estimates on the Green's function and the moment condition, we again get

$$
|F(x)| \leq \frac{C|Q|^{1 / n}}{\left|x-y_{Q}\right|^{n+1}},
$$

for $x \in \Omega \backslash 2 Q$, where $y_{Q}$ is the center of $Q$. For such $x$, we can also bound any first derivatives of $u$ by $|Q|^{1 / n}\left|x-y_{Q}\right|^{-n}$, and $u$ itself by $|Q|^{1 / n}\left|x-y_{Q}\right|^{-n+1}$. However, this does not immediately give us the estimate 5.4 on the integral of $F$. In order to do this, we must switch coordinates.

Let $U=U_{i}$. Recall that in $U$ there exists a system of tangential and normal coordinates $\left(t_{1}, \ldots, t_{n}\right)$. More specifically, we take $\left(t_{1}, \ldots, t_{n-1}\right)$ to be coordinates 
on $\partial \Omega \cap U$ and $t_{n}$ to be the signed geodesic distance to $\partial \Omega$ in the Euclidean metric in $U$. Since $a$ is supported in $\bar{\Omega}$, and its supporting cube is "near" the boundary $\left(Q_{K} \subset U\right)$, we may assume that in the new coordinates it is supported in the cube

$$
\widetilde{Q}=[-\delta, \delta]^{n-1} \times[0,2 \delta],
$$

where $\delta$ is proportional to the sidelength of the original supporting cube (the constants of proportionality depending only on the change of coordinates.) We can now set

$$
\widetilde{Q_{k}}=\left[-2^{k} \delta, 2^{k} \delta\right]^{n-1} \times\left[0,2^{k+1} \delta\right] \subset U_{i}
$$

for $1 \leq k \leq K$, which would allow us to continue as in Case 1, provided we can get the estimate 5.4 .

In $U$, we can rewrite $F$ as

$$
F=\sum_{1 \leq i, m \leq n} a_{i, m} \frac{\partial^{2} u}{\partial t_{i} \partial t_{m}}+\sum_{1 \leq i \leq n} b_{i} \frac{\partial u}{\partial t_{i}}
$$

for some smooth functions $a_{i, m}$ and $b_{i}$. Thus to bound the integral of $F$, it suffices to bound the integrals of all first and second derivatives of $u$ in the coordinates $t_{i}$.

We do this first for the Dirichlet problem. To bound the integral of the first derivatives, we can use the fundamental theorem of calculus and the values of $u$ on the boundary of $\widetilde{Q_{K}}$ to get

$$
\left|\int_{Q_{K}} \frac{\partial u}{\partial t_{i}}(t) d t\right|=\left|\int\left\{\int \frac{\partial u}{\partial t_{i}} d t_{i}\right\} d t^{\prime}\right| \leq C|Q|^{1 / n} .
$$

Note that when $i=n$, this involves the Dirichlet boundary conditions.

For the second derivatives, we again use the bound on the first derivatives of $u$ on the boundary of $\widetilde{Q_{K}}$ to get

$$
\left|\int_{Q_{K}} \frac{\partial^{2} u}{\partial t_{j} \partial t_{l}}(t) d t\right| \leq C|Q|^{1 / n}
$$

as long as either $j<n$ or $l<n$.

In order to get an estimate for $\frac{\partial^{2} u}{\partial t_{n}^{2}}$, we need to use the fact that $\Delta u=a$ in $\Omega$. In the new coordinates, since we chose $t_{n}$ to be the signed geodesic distance to $\partial \Omega$ in the Euclidean coordinates, this translates into

$$
\frac{\partial^{2} u}{\partial t_{n}^{2}}+\sum_{1 \leq i, m \leq n-1} c_{i, m} \frac{\partial^{2} u}{\partial t_{i} \partial t_{m}}+\sum d_{i} \frac{\partial u}{\partial t_{i}}=a,
$$

where $c_{i, m}$ and $d_{i}$ are smooth functions in $U$. Combined with the approximate moment condition on $a$, this gives the desired estimate on the integral of $\frac{\partial^{2} u}{\partial t_{n}^{2}}$, and hence on the integral of $F$ (estimate 5.4).

For the Neumann problem, we are no longer able to estimate the integral of $\frac{\partial u}{\partial t_{n}}$ on $Q_{K}$, as above. However, we can use the Neumann boundary conditions to estimate the integral of $\frac{\partial^{2} u}{\partial t_{n}^{2}}$, as long as $\left.\frac{\partial}{\partial t_{n}}\right|_{\partial \Omega}=-\vec{n}$, where $\vec{n}$ is the outward normal vector on the boundary. Then we can proceed to estimate the integral of $\frac{\partial u}{\partial t_{n}}$ on $Q_{K}$ by solving for $\frac{\partial u}{\partial t_{n}}$ in the equation $\Delta u=a$. We need to make sure that the coefficient of $\frac{\partial}{\partial t_{n}}$ in the expression for the Laplacian is non-vanishing. This coefficient turns out to be the Euclidean Laplacian of $t_{n}, \Delta t_{n}$. But this can always be taken to be non-vanishing, if necessary by replacing $t_{n}$ with $1-e^{-t_{n}}$. These modifications give estimate 5.4 for the Neumann problem. 
Now we can proceed exactly as in Case 1 to get an atomic decomposition for $F$ with atoms supported entirely in $\bar{\Omega}$. These will be $h_{z}^{1}$ atoms with respect to the new coordinates $\left(t_{1}, \ldots, t_{n}\right)$ in $U$, and therefore when we pull back to the original coordinates, they will become modified $h_{z}^{1}$ atoms.

Thus in all three cases we have exhibited an atomic decomposition for $F$ with modified $h_{z}^{1}$ atoms, proving that $F \in h_{z}^{1}(\bar{\Omega})$ with bounded norm.

5.1. BMO regularity. We now come to the question of BMO regularity. First we have to consider the appropriate BMO spaces. We begin by recalling the definition of the local BMO, as defined in [G] (Section 5). We shall call this space bmo $\left(\mathbf{R}^{n}\right)$.

Definition 5.3. A locally integrable functions $g$ is said to belong to $\operatorname{bmo}\left(\mathbf{R}^{n}\right)$ if

$$
\|g\|_{\mathrm{bmo}}=\sup _{|Q|<1} \frac{1}{|Q|} \int_{Q}\left|g(x)-g_{Q}\right| d x+\sup _{|Q| \geq 1} \frac{1}{|Q|} \int_{Q}|g(x)| d x<\infty,
$$

where the suprema are taken over all cubes $Q \subset \mathbf{R}^{n}$ with sides parallel to the axes. Here $g_{Q}$ denotes the mean value of $g$ over $Q$. We call bmo $\left(\mathbf{R}^{n}\right)$ the space of such functions, with norm given by $\|\cdot\|_{\text {bmo }}$.

We can now define a subspace of this space which turns out to be the dual of $h_{d}^{1}$.

Definition 5.4. Let $\Omega$ be a bounded domain with smooth boundary. The space $\mathrm{bmo}_{z}(\bar{\Omega})$ is defined to be the subspace of $\operatorname{bmo}\left(\mathbf{R}^{n}\right)$ consisting of those elements which are supported on $\bar{\Omega}$, i.e.

$$
\operatorname{bmo}_{z}(\bar{\Omega})=\left\{g \in \operatorname{bmo}\left(\mathbf{R}^{n}\right): g=0 \text { on } \mathbf{R}^{n} \backslash \bar{\Omega}\right\},
$$

with

$$
\|g\|_{\mathrm{bmo}_{z}(\bar{\Omega})} \stackrel{\text { def }}{=}\|g\|_{\mathrm{bmo}\left(\mathbf{R}^{n}\right)} .
$$

Theorem $5.5([\mathrm{M}],[\mathrm{C}])$. The space $\operatorname{bmo}_{z}(\bar{\Omega})$ is the dual of $h_{d}^{1}(\bar{\Omega})$.

Remarks. 1. A global version of $\mathrm{bmo}_{z}$ was defined by Miyachi [M], and the local version was defined in $[\mathrm{C}]$, both using a different definition which involved distinguishing between type (a) and type (b) cubes. However, the remark following the proof of Theorem 4 in [M], and part (1) of Proposition 2.2 in $[\mathrm{C}]$, show that this definition is equivalent.

2. The duality theorem follows from Theorem 2 in $[\mathrm{M}]$ and Theorem 2.3 in $[\mathrm{C}]$, after observing that the space $h_{d}^{1}(\bar{\Omega})$ is in fact the same as the space $h_{r}^{1}(\Omega)$ defined in [CKS] (see Proposition 6.4 in Section 6.)

Next, we define the BMO space corresponding to $h_{z}^{1}$.

Definition 5.6. Let $\Omega$ be a bounded domain with smooth boundary. A locally integrable functions $g$ is said to belong to $\operatorname{bmo}_{r}(\Omega)$ if

$$
\|g\|_{\mathrm{bmo}_{r}}=\sup _{|Q|<1} \frac{1}{|Q|} \int_{Q}\left|g(x)-g_{Q}\right| d x+\sup _{|Q| \geq 1} \frac{1}{|Q|} \int_{Q}|g(x)| d x<\infty,
$$

where the suprema are taken over all cubes $Q \subset \Omega$. Here $g_{Q}$ denotes the mean value of $g$ over $Q$.

We call $\mathrm{bmo}_{r}(\Omega)$ the space of such functions, with norm given by $\|\cdot\|_{\mathrm{bmo}_{r}}$. 
Remarks. 1. This definition was introduced in $[\mathrm{C}]$ for a bounded Lipschitz domain $\Omega$. A similar notion is found in [JSW], where the local bmo space $\operatorname{bmo}(F)$ associated to a closed " $d$-set" $F$ is defined by using essentially the same norm, except the cubes are replaced with balls centered inside the set.

2. This space is a local version of the $\operatorname{space} \operatorname{BMO}(\mathcal{D})$ defined by Jones $([\mathrm{J}])$ for a connected open set $\mathcal{D}$, using the norm

$$
\|g\|_{*, \mathcal{D}}=\sup _{Q \subset \mathcal{D}} \frac{1}{|Q|} \int_{Q}\left|g(x)-g_{Q}\right| d x .
$$

3. It should be noted that $\operatorname{bmo}_{z}(\Omega) \subset \mathrm{bmo}_{r}(\Omega)$, and simple examples show that this inclusion is strict.

We now state the duality result:

Theorem 5.7 ([JSW], $[\mathrm{C}])$. The space $\mathrm{bmo}_{r}(\Omega)$ is the dual of $h_{z}^{1}(\bar{\Omega})$.

Remarks. 1. Jonsson, Sjögren and Wallin (see [JSW], Theorem 4.2) prove this theorem for a closed " $d$-set" $F$ having the Markov property, namely they show that $\operatorname{bmo}(F)$ is the dual of the Hardy space $h^{1}(F)$. As mentioned above (see Remark 1 following Definition 1.2), this is the same as $h_{z}^{1}(\bar{\Omega})$ when $\Omega$ is a smoothly bounded domain.

2. In ([C], Theorems 2.1,3.3) it is shown that for a bounded Lipschitz domain in $\mathbf{R}^{n}, \mathrm{bmo}_{r}(\Omega)$ is the dual of the quotient space $h_{z}^{1}(\Omega)$, as defined in [CKS] (see Remark 2 following Definition 1.2). When $p=1$, this quotient space is the same as $h_{z}^{1}(\bar{\Omega})$, since there are no nonzero $h^{1}$ functions which are supported in $\bar{\Omega}$ and vanish on $\Omega$.

In view of the duality theorems, we want to use our $h_{d}^{1}$ and $h_{z}^{1}$ regularity results for the Dirichlet and Neumann problems to prove similar regularity results for $\mathrm{bmo}_{z}$ and bmo $_{r}$. We first have to define the operators $\frac{\partial^{2} \mathbf{G}}{\partial x_{j} \partial x_{l}}$ and $\frac{\partial^{2} \widetilde{\mathbf{G}}}{\partial x_{j} \partial x_{l}}$ on these spaces.

Note that the spaces $\operatorname{bmo}_{z}(\bar{\Omega})$ and $\operatorname{bmo}_{r}(\Omega)$ are subsets of $L^{2}(\Omega)$. In fact, by the John-Nirenberg inequality, since $\mathrm{bmo}_{z}(\bar{\Omega}) \subset \operatorname{bmo}\left(\mathbf{R}^{n}\right) \subset \operatorname{BMO}\left(\mathbf{R}^{n}\right)$ (see $[\mathrm{G}]$, Corollary 1 in Section 4 ) and $\Omega$ is bounded,

$$
\|g\|_{\mathrm{bmo}_{z}(\bar{\Omega})} \geq C\|g\|_{L^{q}(\Omega)}
$$

for all $q<\infty$. Similarly, we have

$$
\|g\|_{\mathrm{bmo}_{r}(\Omega)} \geq C\|g\|_{L^{q}(\Omega)}
$$

(see [C], Lemma 1.6, and the proof of Theorem 4.2 in [JSW]). Thus we can define the operators $\frac{\partial^{2} \mathbf{G}}{\partial x_{j} \partial x_{l}}$ and $\frac{\partial^{2} \widetilde{\mathbf{G}}}{\partial x_{j} \partial x_{l}}$ on $\operatorname{bmo}_{z}(\bar{\Omega})$ and $\operatorname{bmo}_{r}(\Omega)$, in the $L^{2}$ sense.

We have the following regularity results:

Theorem 5.8. If $\Omega$ is as above, and $\mathbf{G}$ is the solution operator for the Dirichlet problem, then for $1 \leq j, l \leq n, \frac{\partial^{2} \mathbf{G}}{\partial x_{j} \partial x_{l}}$ is a bounded operator from $\operatorname{bmo}_{z}(\bar{\Omega})$ to $\mathrm{bmo}_{z}(\bar{\Omega})$ and from $\mathrm{bmo}_{r}(\Omega)$ to $\mathrm{bmo}_{r}(\Omega)$.

Theorem 5.9. If $\Omega$ is as above, and $\widetilde{\mathbf{G}}$ is the solution operator for the Neumann problem, then for $1 \leq j, l \leq n, \frac{\partial^{2} \widetilde{\mathbf{G}}}{\partial x_{j} \partial x_{l}}$ is a bounded operator from $\operatorname{bmo}_{r}(\Omega)$ to $\operatorname{bmo}_{r}(\Omega)$.

Before proceeding with the proofs, we will state and prove a couple of useful lemmas. 
Lemma 5.10. If $T: L^{2}(\Omega) \rightarrow L^{2}(\Omega)$ and the kernel $K(x, y)$ of $T$ satisfies

$$
|K(x, y)| \leq C|x-y|^{-(n-1)}
$$

for all $x \neq y$, then $T$ is bounded from $\mathrm{bmo}_{z}(\bar{\Omega})$ to $\mathrm{bmo}_{z}(\bar{\Omega})$ and from $\mathrm{bmo}_{r}(\Omega)$ to $\mathrm{bmo}_{r}(\Omega)$.

Proof. For $q>n$, if $\frac{1}{q}+\frac{1}{q^{\prime}}=1$, then $q^{\prime}<\frac{n}{n-1}$ and

$$
\int_{\Omega} K(x, y)^{q^{\prime}} d y<\infty .
$$

Hence $T$ is bounded from $L^{q}(\Omega)$ to $L^{\infty}$. Thus for $g \in \operatorname{bmo}_{z}(\bar{\Omega})$,

$$
\|T(g)\|_{\mathrm{bmo}_{z}} \leq\|T(g)\|_{\infty} \leq C\|g\|_{L^{q}} \leq C\|g\|_{\mathrm{bmo}_{z}}
$$

where the last inequality follows from the John-Nirenberg inequality (see the remark preceding Theorem 5.8). Similarly, we get the boundedness on $\mathrm{bmo}_{r}$.

Lemma 5.11. The spaces $\operatorname{bmo}_{z}(\bar{\Omega})$ and $\operatorname{bmo}_{r}(\Omega)$ are closed under multiplication by smooth functions, i.e. if $g \in \mathrm{bmo}_{z}(\bar{\Omega})$ and $\varphi \in \mathcal{C}^{1}(\bar{\Omega})$, then $\varphi g \in \mathrm{bmo}_{z}(\bar{\Omega})$ and

$$
\|\varphi g\|_{\mathrm{bmo}_{z}} \leq C\|\varphi\|_{\mathcal{C}^{1}}\|g\|_{\mathrm{bmo}_{z}}
$$

and similarly for $\operatorname{bmo}_{r}(\Omega)$.

Proof. To prove this for $\mathrm{bmo}_{z}$, it suffices to prove it for $\operatorname{bmo}\left(\mathbf{R}^{n}\right)$. We begin by noting that the bmo norm only changes by a factor of 2 if in equation 5.5, for each cube $Q$, we replace the mean value $g_{Q}$ by some constant $c_{Q}$. Take $\varphi \in C^{1}$ with compact support. Now for every cube $Q$ with $|Q|<1$, letting $c_{Q}=\varphi\left(x_{Q}\right) g_{Q}$, where $x_{Q}$ is the center of $Q$, we get

$$
\begin{aligned}
\frac{1}{|Q|} \int_{Q}\left|\varphi(x) g(x)-\varphi\left(x_{Q}\right) g_{Q}\right| d x \leq & \frac{1}{|Q|} \int_{Q}\left|\varphi(x) g(x)-\varphi(x) g_{Q}\right| d x \\
& +\frac{1}{|Q|} \int_{Q}\left|\varphi(x) g_{Q}-\varphi\left(x_{Q}\right) g_{Q}\right| d x \\
\leq & \|\varphi\|_{\infty}\|g\|_{\mathrm{bmo}}+C\left|g_{Q}\right|\|\varphi\|_{C^{1}}|Q|^{1 / n} \\
\leq & \|\varphi\|_{\infty}\|g\|_{\mathrm{bmo}}+C\|g\|_{L^{n}}\|\varphi\|_{C^{1}} \\
\leq & C\|\varphi\|_{C^{1}}\|g\|_{\mathrm{bmo}} .
\end{aligned}
$$

Clearly if $|Q| \geq 1$,

$$
\frac{1}{|Q|} \int_{Q}|\varphi(x) g(x)| d x \leq\|\varphi\|_{\infty}\|g\|_{\mathrm{bmo}}
$$

Thus $\varphi g \in$ bmo with norm bounded by $\|\varphi\|_{C^{1}}\|g\|_{\text {bmo. }}$.

The proof for $\mathrm{bmo}_{r}$ is obtained from the proof above by considering only cubes $Q \subset \Omega$.

We are now ready to prove the theorems.

Proof of Theorem 5.8. We will give the proof for $\mathrm{bmo}_{r}$, and indicate where changes need to be made for $\mathrm{bmo}_{z}$.

Set $T_{j, l}=\frac{\partial^{2} \mathbf{G}}{\partial x_{j} \partial x_{l}}$, defined on $L^{2}(\Omega)$. Take $g \in \operatorname{bmo}_{r}(\Omega)$. We want to show $T_{j, l}(g) \in \operatorname{bmo}_{r}(\Omega)$ with $\left\|T_{j, l}(g)\right\|_{\mathrm{bmo}_{r}} \leq C\|g\|_{\mathrm{bmo}_{r}}$.

As usual, we introduce a partition of unity $\left\{\eta_{\mu}\right\}, \mu=0, \ldots, k$, with $\eta_{0}$ supported inside $\Omega$, and $\eta_{\mu}, \mu \geq 1$, supported in an open set $U_{\mu}$, with $\bigcup U_{\mu} \supset \partial \Omega$. In each $U_{\mu}$ 
we assume the existence of a coordinate system $\left(t_{1}, \ldots, t_{n}\right)$, where $\left(t_{1}, \ldots, t_{n-1}\right)$ are tangential coordinates, and $t_{n}$ is the normal variable.

We want to show $\eta_{\mu} T_{j, l}(g) \in \mathrm{bmo}_{r}(\Omega)$ for every $\mu$. We begin with $\mu=0$. Case 0: Interior derivatives. We can write

$$
\eta_{0} T_{j, l}=X Y \mathbf{G},
$$

where $X$ and $Y$ are vector fields with compact support in $\Omega$ (i.e. take $X=\eta_{0} \frac{\partial}{\partial x_{j}}$, $Y=\tilde{\eta} \frac{\partial}{\partial x_{l}}$ with $\tilde{\eta}=1$ in the support of $\eta_{0}$ ).

Note that the operator $\mathbf{G} X Y$ is bounded on $\operatorname{bmo}_{r}(\Omega)$, since for $g \in \operatorname{bmo}_{r}(\Omega)$ and $a$ an $h_{z}^{1}$ atom, we have

$$
\begin{aligned}
|\langle\mathbf{G} X Y g, a\rangle| & =\left|\left\langle g, Y^{*} X^{*} \mathbf{G} a\right\rangle\right| \\
& \leq\|g\|_{\mathrm{bmo}_{r}}\left\|Y^{*} X^{*} \mathbf{G} a\right\|_{h_{z}^{1}} \\
& \leq C\|g\|_{\mathrm{bmo}_{r}}
\end{aligned}
$$

by Theorem 5.1. The same proof applies to $\mathrm{bmo}_{z}$ by using Lemma 3.5.

Furthermore, by the local smoothing properties of $\mathbf{G}$, the operator $X Y \mathbf{G}-\mathbf{G} X Y$ is smoothing of order 1 , hence satisfies the hypothesis of Lemma 5.10, and thus is bounded on $\operatorname{bmo}_{r}(\Omega)$. This shows $X Y \mathbf{G}=\eta_{0} T_{j, l}$ is bounded on $\mathrm{bmo}_{r}(\Omega)$.

We now look at the cases where $\mu \geq 1$. Let $U=U_{\mu}$. Then we have that

$$
\eta_{\mu} T_{j, l}=X Y \mathbf{G}
$$

where now $X$ and $Y$ are vector fields with supports in $U$. Recalling the coordinates $\left(t_{1}, \ldots, t_{n}\right)$ defined in $U$, we can rewrite $X$ and $Y$ in terms of the vector fields $\frac{\partial}{\partial t_{i}}$, with smooth coefficients. By Lemma 5.11, it suffices to consider separately the following three cases.

Case 1: Tangential derivatives. We assume that $X$ and $Y$ are tangential vector fields supported in $U$. Then again we have that the operator $\mathbf{G} X Y$ is bounded on $\mathrm{bmo}_{r}(\Omega)$, since we can integrate by parts as in Case 0. Thus it remains to show that the operator $X Y \mathbf{G}-\mathbf{G} X Y$ satisfies the hypothesis of Lemma 5.10. We will show that it is a smoothing operator of order 1.

As in the proof of estimate 3.1 in Section 3, write

$$
\mathbf{G}=\mathbf{E}+\mathbf{H},
$$

where the operator $\mathbf{E}$ is convolution with the Newtonian potential $E$, and $-\mathbf{H}=$ PRE, the Poisson integral of the restriction of $\mathbf{E}$ to the boundary. Now $\mathbf{E}$ is smoothing of order 2, so its commutator with a differential operator of order 2 is smoothing of order 1. As for $\mathbf{P}$, since $X$ and $Y$ are tangential, we have that

$$
X Y \mathbf{P}-\mathbf{P} X Y=\mathbf{P}^{\prime} X+\mathbf{P}^{\prime \prime} Y+\mathbf{P}^{\prime \prime \prime},
$$

where $\mathbf{P}^{\prime}, \mathbf{P}^{\prime \prime}$ and $\mathbf{P}^{\prime \prime \prime}$ are Poisson type operators of order 0 (on $\partial \Omega$ ) - see [GS], pp. 167-168. Composing this with $\mathbf{R E}$, and noting that $\mathbf{R}$ commutes with $X$ and $Y$, while again the commutator with $\mathbf{E}$ is smoothing of order 1, we get that

$$
\begin{aligned}
X Y \mathbf{H}-\mathbf{H} X Y & =-X Y \mathbf{P R E}+\mathbf{P R E} X Y \\
& =-(X Y \mathbf{P}-\mathbf{P} X Y) \mathbf{R E}-\mathbf{P}(X Y \mathbf{R E}-\mathbf{R E} X Y) \\
& =-\left(\mathbf{P}^{\prime} X+\mathbf{P}^{\prime \prime} Y+\mathbf{P}^{\prime \prime \prime}\right) \mathbf{R E}-\mathbf{P R}(X Y \mathbf{E}-\mathbf{E} X Y),
\end{aligned}
$$

which is smoothing of order 1. 
Case 2: One normal derivative. We assume $X$ is a tangential vector field and $Y=$ $\frac{\partial}{\partial t_{n}}$. Denote $Y \mathbf{G}$ by $T_{n}$ and the normal derivative by $\frac{\partial}{\partial \rho}$. Then the kernel of $T_{n}$ is $\frac{\partial}{\partial \rho_{x}} G(x, y)$, so we can define the dual operator $T_{n}^{*}$ by

$$
T_{n}^{*}(f)=\int_{\Omega} \frac{\partial}{\partial \rho_{y}} G(y, x) f(y) d y .
$$

Consider the operator $X^{*} T_{n}^{*}$. Using the same techniques as in Case 2 of the proof of Theorem 5.1, namely the cancellation obtained from the tangential derivative, and the size estimates on the kernel and its derivatives (which are the same regardless of whether we differentiate $G(x, y)$ in the $x$ or the $y$ variable), we get that $X^{*} T_{n}^{*}$ is bounded on $h_{z}^{1}$. This shows that the dual operator, $\left(X^{*} T_{n}^{*}\right)^{*}=T_{n} X$, is bounded on bmo $_{r}$. The same techniques apply for the case of $\mathrm{bmo}_{z}$. In fact, when we have one tangential derivative, as in $X^{*} T_{n}^{*}$, we can even show boundedness from $h_{d}^{1}$ to $h_{z}^{1}$, since we gain some cancellation.

Thus it remains to show $X T_{n}-T_{n} X$ is bounded on $b o_{r}$. In light of Lemma 5.10, it suffice to prove that $X T_{n}-T_{n} X$ is smoothing of order 1 .

Again, this follows from the calculus of pseudo-differential and Poisson-type operators. If we write

$$
\mathbf{G}=\mathbf{E}-\mathbf{P R E}
$$

as above, then

$$
T_{n}=\frac{\partial}{\partial \rho} \mathbf{E}-\frac{\partial}{\partial \rho} \mathbf{P R E} .
$$

Again the first term is smoothing of order 1 , and therefore so is its commutator with $X$.

As for the second term, setting $\mathbf{P}_{n}=\frac{\partial}{\partial \rho} \mathbf{P}$, we get a Poisson-type operator of order 1 . Thus its commutator with the tangential vector field $X$ also gives a Poissontype operator of order 1 . Composing with $\mathbf{R E}$, and recalling that $\mathbf{R}$ commutes with $X$, give

$$
\begin{aligned}
X \mathbf{P}_{n} \mathbf{R E}-\mathbf{P}_{n} \mathbf{R E} X & =\left(X \mathbf{P}_{n}-\mathbf{P}_{n} X\right) \mathbf{R E}+\mathbf{P}_{n}(X \mathbf{R E}-\mathbf{R E} X) \\
& =\left(X \mathbf{P}_{n}-\mathbf{P}_{n} X\right) \mathbf{R E}+\mathbf{P}_{n} \mathbf{R}(X \mathbf{E}-\mathbf{E} X),
\end{aligned}
$$

which is smoothing of order 1.

Case 3: Two normal derivatives. The boundedness of $\frac{\partial^{2}}{\partial \rho^{2}} \mathbf{G}$ on $\operatorname{bmo}_{r}(\Omega)$ follows from the previous two cases by writing $\frac{\partial^{2}}{\partial \rho^{2}}$ in terms of the Laplacian, purely tangential derivatives, mixed tangential and normal derivatives, and first order terms. The Laplacian composed with $\mathbf{G}$ gives the identity, while the first order derivatives of $\mathbf{G}$ are smoothing of order 1 , and are therefore bounded on $\operatorname{bmo}_{r}(\Omega)$ by Lemma 5.10. Finally, Lemma 5.11 guarantees that multiplication by smooth coefficients does not affect the boundedness on $\mathrm{bmo}_{r}(\Omega)$.

Having proved these four cases, we have concluded the proof of Theorem 5.8.

Proof of Theorem 5.9. The proof follows the same lines as for the Dirichlet problem. After localizing, we reduce to the problem of showing that the operator $X Y \widetilde{\mathbf{G}}$ is bounded on $\operatorname{bmo}_{r}(\Omega)$, where $\widetilde{\mathbf{G}}$ is the solution operator for the Neumann problem, and $X$ and $Y$ are vector fields in $\bar{\Omega}$. Case 0 , where $X$ and $Y$ are supported in the interior of $\Omega$, is exactly the same as for the Dirichlet problem, using the interior regularity for the Neumann problem. Thus we can proceed to the cases where $X$ and $Y$ are supported in a coordinate neighborhood of a point on the boundary. 
In the case where both $X$ and $Y$ are tangential, we use the $h_{z}^{1}$ regularity of the Neumann problem to show that the operator $\widetilde{\mathbf{G}} X Y$ is bounded on $\operatorname{bmo}_{r}(\Omega)$. Thus it remains to show the boundedness of the operator $X Y \widetilde{\mathbf{G}}-\widetilde{\mathbf{G}} X Y$, which we again do using Lemma 5.10, i.e. by showing it is a smoothing operator of order 1.

As in the proof of Claim 4.4 in Section 4, we write

$$
\widetilde{\mathbf{G}}=\mathbf{E}+\widetilde{\mathbf{H}}+S,
$$

where $\widetilde{\mathbf{H}}=-\mathbf{P}\left(Q \mathbf{R} \frac{\partial}{\partial \vec{n}} \mathbf{E}\right), S$ is a smoothing error, and $Q$ is smoothing of order 1 on the boundary. We want to look at $X Y \widetilde{\mathbf{G}}-\widetilde{\mathbf{G}} X Y$, where $X$ and $Y$ are tangential vector fields. Again, the commutator with $\mathbf{E}$ gives us an operator of one degree of smoothing, and similarly for the smoothing error $S$. Thus we need only concern ourselves with $\widetilde{\mathbf{H}}$.

Write

$$
\begin{aligned}
X Y \widetilde{\mathbf{H}}-\widetilde{\mathbf{H}} X Y= & -X Y \mathbf{P}\left(Q \mathbf{R} \frac{\partial}{\partial \vec{n}} \mathbf{E}\right)+\mathbf{P}\left(Q \mathbf{R} \frac{\partial}{\partial \vec{n}} \mathbf{E}\right) X Y \\
= & -(X Y \mathbf{P}-\mathbf{P} X Y) Q \mathbf{R} \frac{\partial}{\partial \vec{n}} \mathbf{E}-\mathbf{P}(X Y Q-Q X Y) \mathbf{R} \frac{\partial}{\partial \vec{n}} \mathbf{E} \\
& -\mathbf{P} Q \mathbf{R}\left(X Y \frac{\partial}{\partial \vec{n}} \mathbf{E}-\frac{\partial}{\partial \vec{n}} \mathbf{E} X Y\right),
\end{aligned}
$$

where we have again used the fact that $\mathbf{R}$ commutes with $X$ and $Y$. From equation 5.7 above, we get that the first term on the right-hand side can be written as

$$
\left(\mathbf{P}^{\prime} X+\mathbf{P}^{\prime \prime} Y+\mathbf{P}^{\prime \prime \prime}\right) Q \mathbf{R} \frac{\partial}{\partial \vec{n}} \mathbf{E}
$$

Since $\frac{\partial}{\partial \vec{n}} \mathbf{E}$ is smoothing of order $1, X Q, Y Q$ and $Q$ are operators of order 0 (or better) on $\partial \Omega$, and $\mathbf{P}^{\prime}, \mathbf{P}^{\prime \prime}$ and $\mathbf{P}^{\prime \prime \prime}$ are Poisson-type operators of order 0 , we get that the first term is smoothing of order 1. Similarly, since $X Y Q-Q X Y$ is of order 0 on $\partial \Omega$, the second term is of the same form, hence smoothing of order 1 . In the third term, the commutator $X Y \frac{\partial}{\partial \vec{n}} \mathbf{E}-\frac{\partial}{\partial \vec{n}} \mathbf{E} X Y$ is of order 0 , but $Q$ is smoothing of order 1 on $\partial \Omega$, so again we get a term which is smoothing of order 1.

In the case of one tangential derivative $(X)$ and one normal derivative $(Y)$, denoting the operator $Y \widetilde{\mathbf{G}}$ by $\widetilde{T}_{n}$, and following the argument of Case 2 in the proof of Theorem 5.8 above, we have to show that the operator $X \widetilde{T}_{n}-\widetilde{T}_{n} X$ is smoothing of order 1. Again we only need to consider the commutator of $X$ with

$$
\frac{\partial}{\partial \rho} \widetilde{\mathbf{H}}=-\mathbf{P}_{n}\left(Q \mathbf{R} \frac{\partial}{\partial \vec{n}} \mathbf{E}\right)
$$

where $\mathbf{P}_{n}$ is the normal derivative of the Poisson operator $\mathbf{P}$. The argument proceeds as for the Dirichlet problem.

Finally, the case of the two normal derivatives is also handled the same way as in the proof of Theorem 5.8, so that we can conclude the proof of Theorem 5.9.

\section{Relations Between the Spaces}

In this section we will discuss the relations between the two spaces $h_{z}^{p}(\bar{\Omega})$ and $h_{d}^{p}(\bar{\Omega})$, as well as their relations to the spaces $h_{z}^{p}(\Omega)$ and $h_{r}^{p}(\Omega)$ considered in [CKS].

We begin by comparing $h_{z}^{p}(\bar{\Omega})$ and $h_{d}^{p}(\bar{\Omega})$. To do so, we must consider elements of $h_{z}^{p}(\bar{\Omega})$ as distributions in $\mathcal{C}_{d}^{\infty \prime}(\bar{\Omega})$. That is, for $f \in h_{z}^{p}(\bar{\Omega})$, we can define a linear 
functional $\tilde{f}$ on $\mathcal{C}_{d}^{\infty}(\bar{\Omega})$ by

$$
\langle\tilde{f}, \varphi\rangle=\langle f, \tilde{\varphi}\rangle,
$$

where $\varphi \in \mathcal{C}_{d}^{\infty}(\bar{\Omega})$, and $\tilde{\varphi} \in \mathcal{S}\left(\mathbf{R}^{n}\right)$ is an extension of $\varphi$. This is independent of the extension because $f$ is supported on the compact set $\bar{\Omega}$, which also shows that $\tilde{f}$ must be continuous on $\mathcal{C}_{d}^{\infty}(\bar{\Omega})$. Thus $f \rightarrow \tilde{f}$ is a mapping from $h_{z}^{p}(\bar{\Omega})$ to $\mathcal{C}_{d}^{\infty \prime}(\Omega)$. The kernel of this map is

$$
h_{0}^{p}(\partial \Omega)=\left\{f \in h^{p}\left(\mathbf{R}^{n}\right):\langle f, \varphi\rangle=0 \forall \varphi \in \mathcal{S} \text { with }\left.\varphi\right|_{\partial \Omega}=0\right\},
$$

which is the space of $h^{p}\left(\mathbf{R}^{n}\right)$ distributions supported on $\partial \Omega$ and having order zero in the normal direction. (This is not to be confused with any $h^{p}$ space that can be defined on $\partial \Omega$ as a manifold.)

We can identify the quotient space $h_{z}^{p}(\bar{\Omega}) / h_{0}^{p}(\partial \Omega)$ with a subspace of $\mathcal{C}_{d}^{\infty \prime}(\Omega)$. As such, we have the following characterization:

Proposition 6.1. For $p<1$,

$$
h_{d}^{p}(\bar{\Omega}) \cong h_{z}^{p}(\bar{\Omega}) / h_{0}^{p}(\partial \Omega)
$$

with comparable norms, i.e. there is a linear operator $T$ from $h_{z}^{p}(\bar{\Omega}) / h_{0}^{p}(\partial \Omega)$ onto $h_{d}^{p}(\bar{\Omega})$ such that for every equivalence class $[f] \in h_{z}^{p}(\bar{\Omega}) / h_{0}^{p}(\partial \Omega)$,

$$
\|T([f])\|_{h_{d}^{p}(\bar{\Omega})} \approx \inf \left\{\|f\|_{h_{z}^{p}(\bar{\Omega})}: f \in[f]\right\} .
$$

Proof. As explained above, since $h_{0}^{p}(\partial \Omega)$ is the kernel of the map $f \rightarrow \tilde{f}$ from $h_{z}^{p}(\bar{\Omega})$ into $\mathcal{C}_{d}^{\infty \prime}(\bar{\Omega})$, we can define $T$ from $h_{z}^{p}(\bar{\Omega})$ by $T([f])=\tilde{f}$, and this is independent of the choice of the representative $f$. Thus to show that the image of this map is contained $h_{d}^{p}(\bar{\Omega})$, it suffices to show that for every $f \in h_{z}^{p}(\bar{\Omega}), \tilde{f} \in h_{d}^{p}(\bar{\Omega})$ and

$$
\|\tilde{f}\|_{h_{d}^{p}} \leq\|f\|_{h_{z}^{p}} .
$$

This can be seen in two ways. Using the maximal function definitions (as in Section 1 ), we see that by extending every normalized $\mathcal{C}_{d}^{\infty}(\bar{\Omega})$ bump function to a normalized bump function in $\mathcal{D}\left(\mathbf{R}^{n}\right)$, we have that

$$
m_{d}(\tilde{f})(x) \leq m(f)(x)
$$

for all $x \in \Omega$; hence $\tilde{f} \in h_{d}^{p}(\bar{\Omega})$ with

$$
\|\tilde{f}\|_{h_{d}^{p}}=\left\|m_{d}(\tilde{f})\right\|_{L^{p}(\Omega)} \leq\|m(f)\|_{L^{p}\left(\mathbf{R}^{n}\right)}=\|f\|_{h_{z}^{p}} .
$$

Alternatively, using the atomic decompositions, it is enough to note that for an $h_{z}^{p}$ atom $a, \tilde{a}=a$ is already an $h_{d}^{p}(\bar{\Omega})$ atom.

Conversely, to show that $T$ is onto and the bounds on the norms can be reversed, we have to show that if $g \in h_{d}^{p}(\bar{\Omega})$, we can write $g=\tilde{f}$ for some $f \in h_{z}^{p}(\bar{\Omega})$, and

$$
\|g\|_{h_{d}^{p}} \geq C \inf _{\tilde{f}=g}\|f\|_{h_{z}^{p}} .
$$

As in Theorem 2.6, write $g=\sum \lambda_{j} a_{j}$, where $a_{j}$ are $h_{d}^{p}$ atoms. Suppose we can show that every $a_{j}=\tilde{f}_{j}$ for some $f_{j} \in h_{z}^{p}(\bar{\Omega})$ with $\left\|f_{j}\right\|_{h_{z}^{p}} \leq C$. Then setting $f=\sum \lambda_{j} f_{j}$ in $\mathcal{S}^{\prime}\left(\mathbf{R}^{n}\right)$, we get that $f \in h_{z}^{p}(\bar{\Omega})$ and $\tilde{f}=\sum \lambda_{j} \tilde{f}_{j}=g$, since the map $f \rightarrow \tilde{f}$ is continuous on $\mathcal{S}^{\prime}\left(\mathbf{R}^{n}\right)$. Furthermore,

$$
\|f\|_{h_{z}^{p}} \leq C\left(\sum\left|\lambda_{j}\right|^{p}\right)^{1 / p}
$$


and each atomic decomposition of $g$ gives rises to such an $f$, so

$$
\inf _{\tilde{f}=g}\|f\|_{h_{z}^{p}} \leq C \inf \left(\sum\left|\lambda_{j}\right|^{p}\right)^{1 / p}=C^{\prime}\|g\|_{h_{d}^{p}} .
$$

Now if $a_{j}$ is a type (a) $h_{d}^{p}$ atom, or an atom supported in a large cube, then it is also an $h_{z}^{p}$ atom, so we can take $f_{j}=a_{j}$. Thus it remains to prove the following:

Lemma 6.2. Suppose $p<1$. If a is a type (b) $h_{d}^{p}$ atom with a small supporting cube (i.e. $\left.|Q|<C_{\Omega}\right)$, then there exists $f \in h_{z}^{p}(\bar{\Omega})$ such that $f-a \in h_{0}^{p}(\partial \Omega)$ and

$$
\|f\|_{h_{z}^{p}(\bar{\Omega})} \leq C
$$

with $C$ independent of $a$.

In particular, this shows that $a=\tilde{f}$, since

$$
\langle\tilde{f}-a, \varphi\rangle=\langle f-a, \tilde{\varphi}\rangle=0
$$

for all $\varphi \in \mathcal{C}_{d}^{\infty}(\bar{\Omega})$.

Proof of lemma. Since $a$ is supported in $Q \cap \bar{\Omega}$, where the cube $Q$ is small and near the boundary, we can find a neighborhood $U$ of $\partial \Omega$ containing $Q$, in which there is defined a smooth projection $\pi: U \rightarrow \partial \Omega$.

Define a linear functional $\Lambda$ on $\phi \in \mathcal{S}\left(\mathbf{R}^{n}\right)$ by

$$
\langle\Lambda, \phi\rangle=\int a(y) \phi(\pi(y)) d y .
$$

Since $\pi$ is smooth, $\Lambda$ is continuous, hence a tempered distribution. Furthermore, $\langle\Lambda, \varphi\rangle=0$ whenever $\left.\varphi\right|_{\partial \Omega}=0$.

We claim that $\Lambda \in h^{p}\left(\mathbf{R}^{n}\right)$. To see this, we look at the grand maximal function $m(\Lambda)$, as in Definition 1.1. Take $x \in \mathbf{R}^{n}$, and let $\phi_{t}^{x}$ be a normalized bump function supported in a ball of radius $R \leq 1$ containing $x$. Then

$$
\begin{aligned}
\left|\left\langle\Lambda, \phi_{t}^{x}\right\rangle\right| & =\left|\int_{Q} a(y) \phi_{t}^{x}(\pi(y)) d y\right| \\
& \leq|Q|^{-1 / p}\left\|\phi_{t}^{x}\right\|_{\infty}\left|\left\{y \in \operatorname{supp}(a): \pi(y) \in \operatorname{supp}\left(\phi_{t}^{x}\right)\right\}\right| \\
& \leq C|Q|^{-1 / p}\left\|\phi_{t}^{x}\right\|_{\infty} \operatorname{diam}(Q) t^{n-1} \\
& \leq C^{\prime}|Q|^{-1 / p+1 / n} t^{-1} \\
& \leq C^{\prime \prime}|Q|^{-1 / p+1 / n}(\operatorname{dist}(x, \partial \Omega))^{-1}
\end{aligned}
$$

since we must have $2 t \geq \operatorname{dist}(x, \partial \Omega)$ in order for $\phi_{t}^{x}(\pi(y)) \neq 0$ for some $y$.

If we now vary $\phi_{t}^{x}$, we see that the maximal function $m(\Lambda)$ is supported in a tubular neighborhood of "radius" 2 around $\partial \Omega$. Thus

$$
\begin{aligned}
\int_{\mathbf{R}^{n}} m(\Lambda)(x)^{p} d x & \leq C|Q|^{-1+p / n} \int_{\{x: \operatorname{dist}(x, \partial \Omega) \leq 2\}}(\operatorname{dist}(x, \partial \Omega))^{-p} d x \\
& \leq C_{Q, \Omega}
\end{aligned}
$$

since $p<1$ and $\Omega$ is bounded. Thus $\Lambda \in h^{p}\left(\mathbf{R}^{n}\right)$, and since it is supported on $\partial \Omega$ and has order zero, $\Lambda \in h_{0}^{p}(\partial \Omega)$. Note that the $h^{p}$ norm of $\Lambda$ depends on $a$, and may blow up as the supporting cube of $a$ shrinks.

Now let $f=a-\Lambda$ in $\mathcal{S}^{\prime}\left(\mathbf{R}^{n}\right)$. Since $a \in h^{p}\left(\mathbf{R}^{n}\right)$ and $\Lambda \in h^{p}\left(\mathbf{R}^{n}\right)$, we have $f \in h^{p}\left(\mathbf{R}^{n}\right)$. Also, $f=0$ on $\mathbf{R}^{n} \backslash \bar{\Omega}$, so $f \in h_{z}^{p}(\bar{\Omega})$. We want to show its norm is bounded by a constant independent of $a$. 
Let us compute the local grand maximal function $m(f)$. For $x \in 2 Q$, write

$$
m(f)(x) \leq m(a)(x)+m(\Lambda)(x) .
$$

Now we know from the size condition on $a$ and the $L^{2}$ boundedness of the maximal function that

$$
\begin{aligned}
\int_{2 Q} m(a)^{p} & \leq C\left(\int_{2 Q}|a|^{2}\right)^{p / 2}|Q|^{1-p / 2} \\
& \leq C .
\end{aligned}
$$

As for $\Lambda$, we have from above that

$$
\begin{aligned}
\int_{2 Q} m(\Lambda)^{p}(x) d x & \leq C|Q|^{-1+p / n} \int_{2 Q}(\operatorname{dist}(x, \partial \Omega))^{-p} d x \\
& \leq C^{\prime}|Q|^{-1+p / n} \operatorname{diam}(Q)^{1-p} \operatorname{diam}(Q)^{n-1} \\
& \leq C^{\prime \prime} .
\end{aligned}
$$

When $x \notin 2 Q$, take $\phi_{t}^{x}$ as above and write

$$
\begin{aligned}
\left\langle f, \phi_{t}^{x}\right\rangle & =\int_{Q} a(y) \phi_{t}^{x}(y) d y-\int_{Q} a(y) \phi_{t}^{x}(\pi(y)) d y \\
& =\int_{Q} a(y)\left[\phi_{t}^{x}(y)-\phi_{t}^{x}(\pi(y))\right] d y .
\end{aligned}
$$

But the function $\varphi_{t}^{x}(y)=\phi_{t}^{x}(y)-\phi_{t}^{x}(\pi(y))$ is in $\mathcal{C}_{d}^{\infty}(\bar{\Omega})$ (where we might have to use a cutoff function supported in $U$ in order to define $\varphi$ for all $y$ ). Thus by the moment conditions on $a$,

$$
\begin{aligned}
\left|\left\langle f, \phi_{t}^{x}\right\rangle\right| & \leq\left\|\varphi_{t}^{x}\right\|_{\mathcal{C}^{N_{p}+1}(Q)}|Q|^{\nu_{p}} \\
& \leq C_{\pi}\left\|\phi_{t}^{x}\right\|_{\mathcal{C}^{N_{p}+1}(Q)}|Q|^{\nu_{p}} \\
& \leq C t^{-n-\left(N_{p}+1\right)}|Q|^{\nu_{p}} \\
& \leq C^{\prime}(\operatorname{dist}(x, Q))^{-n-\left(N_{p}+1\right)}|Q|^{\left(N_{p}+1\right) / n+1-1 / p} .
\end{aligned}
$$

Note that for $\left\langle f, \varphi_{t}^{x}\right\rangle \neq 0$ we must have either $2 t \geq \operatorname{dist}(x, Q)$ or $2 t \geq \operatorname{dist}(x, \pi(Q))$, and since $x \notin 2 Q$ and $Q$ is a type (b) cube, this gives $t \geq C \operatorname{dist}(x, Q)$ for some constant $C$.

Thus

$$
\begin{aligned}
\int_{x \notin 2 Q} m(f)(x)^{p} d x & \leq C|Q|^{p\left(N_{p}+1\right) / n+p-1} \int_{x \notin 2 Q}(\operatorname{dist}(x, Q))^{-n p-\left(N_{p}+1\right) p} \\
& \leq C^{\prime}|Q|^{p\left(N_{p}+1\right) / n+p-1} \operatorname{diam}(Q)^{n-n p-\left(N_{p}+1\right) p} \\
& \leq C^{\prime \prime}
\end{aligned}
$$

since $n p+\left(N_{p}+1\right) p>n p+n(1 / p-1) p=n$.

We have now shown that

$$
\|f\|_{h_{z}^{p}}=\|m(f)\|_{L^{p}\left(\mathbf{R}^{n}\right)} \leq C,
$$

which concludes the proof of Lemma 6.2.

By the atomic decomposition, this also completes the proof of Proposition 6.1. 
Note that Proposition 6.1 applies only to $p<1$. For the case $p=1$, the situation is different. Since elements of $h^{1}\left(\mathbf{R}^{n}\right)$ are functions, the space $h_{0}^{1}(\partial \Omega)$ consists only of the zero function. Thus the quotient space $h_{z}^{1}(\bar{\Omega}) / h_{0}^{1}(\partial \Omega)$ is just $h_{z}^{1}(\bar{\Omega})$, the $h^{1}$ functions supported on $\bar{\Omega}$. We claim that this space is different from $h_{d}^{1}(\bar{\Omega})$.

By the atomic decomposition (Theorem 2.6), we know that the elements of $h_{d}^{1}(\bar{\Omega})$ are also functions (since the sums converge in $L^{1}$ ). We want to characterize these functions.

In order to do this, we recall the space $h_{r}^{p}(\Omega)$, defined in [M] and [CKS]. Let $\mathcal{D}(\Omega)$ denote the space of $\mathcal{C}^{\infty}$ functions with compact support in $\Omega$, and let $\mathcal{D}^{\prime}(\Omega)$ denote its dual, the space of distributions on $\Omega$.

Definition 6.3. The space $h_{r}^{p}(\Omega)$ consists of elements of $\mathcal{D}^{\prime}(\Omega)$ which are the restrictions to $\Omega$ of elements of $h^{p}\left(\mathbf{R}^{n}\right)$, i.e.

$$
h_{r}^{p}(\Omega)=h^{p}\left(\mathbf{R}^{n}\right) /\left\{f \in h^{p}\left(\mathbf{R}^{n}\right): f=0 \text { on } \Omega\right\},
$$

equipped with the quotient norm

$$
\|[f]\|_{h_{r}^{p}(\Omega)}=\inf \left\{\|f\|_{h^{p}\left(\mathbf{R}^{n}\right)}: f \in[f]\right\} .
$$

Again it is clear that when $p=1$, this is a space of functions, since elements of $h_{r}^{1}(\Omega)$ are just restrictions to $\Omega$ of functions in $h^{1}\left(\mathbf{R}^{n}\right)$. Thus we can state:

Proposition 6.4. For $\frac{n}{n+1}<p \leq 1$,

$$
h_{d}^{p}(\bar{\Omega}) \cong h_{r}^{p}(\Omega)
$$

Furthermore, considered as subspaces of $L^{1}(\Omega)$, we have

$$
h_{z}^{1}(\bar{\Omega}) \varsubsetneqq h_{d}^{1}(\bar{\Omega})=h_{r}^{1}(\Omega) .
$$

Proof. The equivalence of $h_{d}^{p}(\bar{\Omega})$ and $h_{r}^{p}(\Omega)$ follows immediately from the atomic decompositions. From $[\mathrm{M}]$ (Theorem 1) and [CKS] (Theorem 2.7), we know that every $f \in h_{r}^{p}(\Omega)$ has a decomposition

$$
f=\sum \lambda_{j} a_{j}
$$

in $\mathcal{D}^{\prime}(\Omega)$, where the $a_{j}$ are either $h^{p}\left(\mathbf{R}^{n}\right)$ atoms supported in type (a) cubes, or atoms supported in type (b) cubes and satisfying a size condition, but no moment condition. Note that these are essentially type (a) and type (b) $h_{d}^{p}$ atoms, since when $\frac{n}{n+1}<p \leq 1$, the moment conditions 2.3 in Definition 2.3 are null (they follow from the size condition by expanding the $\mathcal{C}_{d}^{\infty}$ test function around a point on the boundary). The only difference is that in both $[\mathrm{M}]$ and [CKS], type (b) cubes are assumed to be contained in $\Omega$, and in fact their distance from the boundary is assumed to be proportional to their diameter. However, because of the lack of moment conditions, it can be shown that a type (b) $h_{d}^{p}$ atom can be decomposed into type (b) $h_{r}^{p}$ atoms, as in $[\mathrm{M}]$ and $[\mathrm{CKS}]$ - see [CKS], pp. 295-296, where this is done for $\mathbf{R}_{+}^{n}$.

Note that for this range of $p, h_{r}^{p}(\Omega)$ distributions can be applied to test functions with only one order of vanishing at the boundary, and therefore can be considered as distributions in $\mathcal{C}_{d}^{\infty \prime}(\bar{\Omega})$. Also for this range of $p$, the convergence of the atomic decompositions in $\mathcal{D}^{\prime}(\Omega)$ and in $\mathcal{C}_{d}^{\infty}(\bar{\Omega})$ is equivalent. Thus we have the same atomic decomposition for $h_{r}^{p}(\Omega)$ and $h_{d}^{p}(\bar{\Omega})$, considered as subspaces of $\mathcal{C}_{d}^{\infty \prime}(\bar{\Omega})$, which means that these two spaces are the same, with equivalent norms. 
The fact that $h_{z}^{1}(\bar{\Omega})$ is a subset $h_{r}^{1}(\Omega)$ is obvious, since every function $f \in h_{z}^{1}(\bar{\Omega})$ can be extended by 0 to a function in $h^{1}\left(\mathbf{R}^{n}\right)$, and the norm is the same. Thus $f \in h_{r}^{1}(\Omega)$ and

$$
\|f\|_{h_{r}^{1}} \leq\|f\|_{h_{z}^{1}}
$$

since the $h_{r}^{1}$ norm is the infimum of the $h^{1}\left(\mathbf{R}^{n}\right)$ norms of all possible extensions of $f$.

To see that $h_{z}^{1}(\bar{\Omega})$ is a strict subset of $h_{r}^{1}(\Omega)$, we will construct a function $f \in$ $h_{r}^{1}(\Omega) \backslash h_{z}^{1}(\bar{\Omega})$. By translating and rescaling, we may assume $0 \in \partial \Omega$ and the cone

$$
\Gamma=\left\{x=\left(x^{\prime}, x_{n}\right): 2\left|x^{\prime}\right| \leq x_{n},\left|x_{n}\right| \leq 1\right\} \subset \Omega .
$$

For $j \geq 1$, set

$$
Q_{j}=\left[-2^{-j-1}, 2^{-j-1}\right]^{n-1} \times\left[2^{-j}, 2^{-j+1}\right],
$$

and

$$
a_{j}=\left|Q_{j}\right|^{-1} \chi_{Q_{j}} .
$$

Then $a_{j}$ are type (b) $h_{r}^{1}$ atoms. Let

$$
f=\sum_{j=1}^{\infty} \frac{1}{j^{2}} a_{j} .
$$

Then $f \in h_{r}^{1}(\Omega)$.

It is also easy to see that $m(f)(x) \geq \frac{c}{|x|^{n} \log (1 /|x|)}$, for $x \in \Gamma,|x|<1 / 2$, and hence, $f \notin h_{z}^{1}(\Omega)$. This concludes the proof of Proposition 6.4.

We may now ask what are the relations between the spaces $h_{z}^{p}(\bar{\Omega})$ and $h_{d}^{p}(\bar{\Omega})$ and the spaces $h_{r}^{p}(\Omega)$ when $p$ is small. Recall that in [CKS], in order to relate $h_{z}^{p}$ to $h_{r}^{p}$, the quotient space

$$
h_{z}^{p}(\Omega)=h_{z}^{p}(\bar{\Omega}) /\left\{f \in h_{z}^{p}(\bar{\Omega}): f=0 \text { on } \Omega\right\}
$$

was introduced (see Remark 2 following Definition 1.2.) Note that in this case, the null space

$$
h^{p}(\partial \Omega)=\left\{f \in h_{z}^{p}(\bar{\Omega}): f=0 \text { on } \Omega\right\}
$$

is the whole space of $h^{p}$ distributions supported on $\partial \Omega$, which is strictly larger than $h_{0}^{p}(\partial \Omega)$ when $p<\frac{n}{n+1}$.

We can identify $h_{z}^{p}(\Omega)$ with a set of distributions in $\mathcal{D}^{\prime}(\Omega)$, and equipped with the quotient norm, it is clearly a subspace of $h_{r}^{p}(\Omega)$.

By analogy, we can define

$$
h_{d}^{p}(\Omega)=h_{d}^{p}(\bar{\Omega}) /\left\{f \in h_{d}^{p}(\bar{\Omega}): f=0 \text { on } \Omega\right\},
$$

where $f=0$ on $\Omega$ for $f \in \mathcal{C}_{d}^{\infty \prime}$ means $\langle f, \varphi\rangle=0$ whenever $\varphi \in \mathcal{C}_{d}^{\infty}(\bar{\Omega})$ has compact support in $\Omega$. In other words, the null space is the space of distributions in $h_{d}^{p}(\bar{\Omega})$ which are supported on $\partial \Omega$.

Again it is easy to see that elements of $h_{d}^{p}(\Omega)$ can be identified with distributions on $\Omega$, since every $f \in \mathcal{C}_{d}^{\infty}(\bar{\Omega})$ immediately induces a distribution in $\mathcal{D}^{\prime}(\Omega)$, which is zero precisely when $\langle f, \varphi\rangle=0$ for every smooth $\varphi$ with compact support in $\Omega$.

Proposition 6.5. Considered as subspaces of $\mathcal{D}^{\prime}(\Omega)$, when $p<1$,

$$
h_{z}^{p}(\Omega)=h_{d}^{p}(\Omega) \subset h_{r}^{p}(\Omega) .
$$

Moreover, for $N=0,1,2, \ldots$ and $\frac{n}{n+N+1}<p<\frac{n}{n+N}$,

$$
h_{z}^{p}(\Omega)=h_{r}^{p}(\Omega) .
$$


Proof. The fact that $h_{z}^{p}(\Omega)=h_{d}^{p}(\Omega)$ as spaces of distributions on $\Omega$ follows almost immediately from Proposition 6.1 and its proof. For consider an equivalence class $[f] \in h_{z}^{p}(\Omega)$. Taking a representative $f \in[f], f \in h_{z}^{p}(\bar{\Omega})$, we can map $f$ to $\tilde{f} \in h_{d}^{p}(\bar{\Omega})$, with

$$
\|\tilde{f}\|_{h_{d}^{p}(\bar{\Omega})} \leq\|f\|_{h_{z}^{p}(\bar{\Omega})} .
$$

But if we also have $g \in[f]$, then $f=g$ in $\mathcal{D}^{\prime}(\Omega)$; hence $\tilde{f}=\tilde{g}$ in $\mathcal{D}^{\prime}(\Omega)$. Thus we can map $[f]$ to the equivalence class $[\tilde{f}]$ in $h_{d}^{p}(\Omega)$, and

$$
\|[\tilde{f}]\|_{h_{d}^{p}(\Omega)}=\inf _{h \in \tilde{f}}\|h\|_{h_{d}^{p}(\bar{\Omega})} \leq \inf _{g \in[f]}\|g\|_{h_{z}^{p}(\bar{\Omega})}=\|[f]\|_{h_{z}^{p}(\Omega)} .
$$

This shows $h_{z}^{p}(\Omega) \subset h_{d}^{p}(\Omega)$ with a bound on the norms.

Conversely, we show in the proof of Proposition 6.1 that every $f \in h_{d}^{p}(\bar{\Omega})$ can be decomposed in $\mathcal{C}_{d}^{\infty}(\bar{\Omega})$ as $g+b$, where $g \in h_{z}^{p}(\bar{\Omega}), b \in h_{0}^{p}(\partial \Omega)$, and the norm $\|f\|_{h_{d}^{p}}$ is equivalent to the infimum of the norms $\|g\|_{h_{z}^{p}}$ over all such decompositions. Since this decomposition holds a fortiori in $\mathcal{D}^{\prime}(\Omega)$, we get that every equivalence class $[f] \in h_{d}^{p}(\Omega)$ is the image of an equivalence class $[g] \in h_{z}^{p}(\Omega)$, with

$$
\|f\|_{h_{d}^{p}(\Omega)} \geq C\|[g]\|_{h_{z}^{p}(\Omega)} .
$$

Thus we have shown that $h_{z}^{p}(\Omega)=h_{d}^{p}(\Omega)$ with equivalent norms.

Since we have already pointed out that $h_{z}^{p}(\Omega) \subset h_{r}^{p}(\Omega)$, we now get that $h_{d}^{p}(\Omega) \subset$ $h_{r}^{p}(\Omega)$. This can also be seen from the atomic decomposition, since type (b) $h_{d}^{p}$ atoms are essentially (or can be decomposed into) type (b) $h_{r}^{p}$ atoms - see the proof of Proposition 6.4.

For the case $\frac{n}{n+N+1}<p<\frac{n}{n+N}$, in light of the atomic decomposition for $h_{r}^{p}$, it suffices to prove that every $h_{r}^{p}$ atom is in $h_{z}^{p}(\Omega)$, with bounded norm. This is obvious for atoms supported in large cubes and for type (a) atoms, since they are already $h_{z}^{p}$ atoms. Thus it remains to show the following:

Lemma 6.6. Suppose $\frac{n}{n+N+1}<p<\frac{n}{n+N}$. Let a be a type (b) $h_{r}^{p}$ atom supported in a cube $Q$ with $|Q|<1$. Then in $\mathcal{D}^{\prime}(\Omega)$,

$$
a=\sum \lambda_{j} a_{j},
$$

where $a_{j}$ are $h_{z}^{p}$ atoms, and

$$
\sum\left|\lambda_{j}\right|^{p} \leq C,
$$

where the constant $C$ is independent of a.

Proof of lemma. By choosing appropriate coordinates, we can assume $0 \in \partial \Omega$ and $a$ is supported in

$$
Q=[-\delta, \delta]^{n-1} \times[(A-1) \delta,(A+1) \delta]
$$

with $\delta<1$ and $A \geq 1$.

Note that $N=N_{p}$, so that the $a_{j}$ (when supported in small cubes) need to satisfy all moment conditions up to order $N$. We proceed by induction.

Let $K \leq N$, and assume $a$ has vanishing moments up to order $K-1$ (no moment condition is assumed when $K=0)$. We will show that we can write $a=\sum \lambda_{j} a_{j}$ with $a_{j}$ having vanishing moments up to order $K$, and $\sum\left|\lambda_{j}\right|^{p} \leq C$. The $a_{j}$ will also satisfy the size condition, and the supporting cubes $Q_{j}$ will satisfy the conditions:

$$
A^{\prime} Q_{j} \subset \Omega
$$


and

$$
2 A^{\prime} Q_{j} \cap \partial \Omega \neq \emptyset,
$$

with $A^{\prime}=(3 A+1) /(A+3) \geq 1$. Thus the new atoms will satisfy the induction hypothesis for the next step.

Let $b_{0}=a$, and for $j=1,2,3, \ldots$, define $b_{j}$ by

$$
b_{j}(x)=2^{j(K+n)} a\left(2^{j} x\right) .
$$

Then $b_{j}$ is supported in

$$
\widetilde{Q_{j}}=\left[-2^{-j} \delta, 2^{-j} \delta\right]^{n-1} \times\left[2^{-j}(A-1) \delta, 2^{-j}(A+1) \delta\right] .
$$

Also,

$$
\int_{\widetilde{Q_{j}}} b_{j}(x) x^{\alpha} d x=2^{j(K-|\alpha|)} \int_{Q} a(x) x^{\alpha} d x,
$$

so $b_{j}$ satisfies the same moment conditions as $a$. Finally,

$$
\left\|b_{j}\right\|_{\infty} \leq 2^{j(K+n)}|Q|^{-1 / p} \text {. }
$$

Now if we set

$$
f_{j}=b_{j}-b_{j+1},
$$

we have that $f_{j}$ is supported in the smallest cube containing $\widetilde{Q_{j}}$ and $\widetilde{Q_{j+1}}$, namely

$$
Q_{j}=\left[-2^{-(j+2)}(A+3) \delta, 2^{-(j+2)}(A+3) \delta\right]^{n-1} \times\left[2^{-(j+1)}(A-1) \delta, 2^{-j}(A+1) \delta\right],
$$

$f_{j}$ satisfies all the moment conditions that $a$ satisfies, and in addition

$$
\begin{aligned}
\int_{Q_{j}} f_{j}(x) x^{\alpha} d x & =\int_{\widetilde{Q_{j}}} b_{j}(x) x^{\alpha} d x-\int{\widetilde{\widetilde{Q_{j+1}}}}_{j+1}(x) x^{\alpha} d x \\
& =2^{j(K-|\alpha|)} \int_{Q} a(x) x^{\alpha} d x-2^{(j+1)(K-|\alpha|)} \int_{Q} a(x) x^{\alpha} d x \\
& =0
\end{aligned}
$$

whenever $|\alpha|=K$. As for size,

$$
\begin{aligned}
\left\|f_{j}\right\|_{\infty} & \leq 2^{j(K+n)}|Q|^{-1 / p} \\
& =C_{n, p, A} 2^{j(K+n-n / p)}\left|Q_{j}\right|^{-1 / p} .
\end{aligned}
$$

Thus we can let

$$
\lambda_{j}=C_{n, p, A} 2^{j(K+n-n / p)}
$$

and

$$
a_{j}=\lambda_{j}^{-1} f_{j}
$$

The $a_{j}$ satisfy the conditions stated above, and

$$
\sum\left|\lambda_{j}\right|^{p}=C_{n, p, A}^{\prime} \sum_{j=0}^{\infty} 2^{j(K+n-n / p) p} \leq C
$$

since $K \leq N<n / p-n$.

It remains to show $a=\sum \lambda_{j} a_{j}$ in $\mathcal{D}^{\prime}(\Omega)$. However, since $a-\sum_{0}^{J} \lambda_{j} a_{j}=b_{J+1}$ is supported in $\widetilde{Q_{J+1}}$, which is contained in smaller and smaller neighborhoods of $\partial \Omega$ as $J$ increases, we have

$$
\lim _{J \rightarrow \infty}\left\langle a-\sum_{0}^{J} \lambda_{j} a_{j}, \psi\right\rangle \rightarrow 0
$$


for every $\psi$ with compact support in $\Omega$ (actually one can show that the vanishing of $\psi$ up to order $K$ at the boundary is sufficient). This completes the proof of the lemma.

As was pointed out, by the atomic decomposition, this also concludes the proof of Proposition 6.5.

The question arises as to the situation for $p=n /(n+N), N=0,1,2, \ldots$ We already saw in Proposition 6.4 that when $p=1, h_{z}^{1}$ is a strict subspace of $h_{d}^{1}=h_{r}^{1}$ (here the distinction between the spaces and their quotients is irrelevant). Thus we have the following:

Problem. Let $p=n /(n+N), N=1,2, \ldots$ Then considered as subspaces of $\mathcal{D}^{\prime}(\Omega)$, is it true that $h_{z}^{p}(\Omega) \neq h_{r}^{p}(\Omega)$ ?

In other words, the question is whether for the "critical" values of $p$, there exists a distribution $f$ on $\Omega$ which is the restriction to $\Omega$ of an element of $h^{p}\left(\mathbf{R}^{n}\right)$, but not the restriction to $\Omega$ of any element of $h^{p}\left(\mathbf{R}^{n}\right)$ which is supported on $\bar{\Omega}$. Intuitively, the idea is that one cannot "correct" the lack of cancellation of $f$ by a distribution supported on $\partial \Omega$. To answer the question, one needs to understand the behavior of the maximal function of distributions supported on $\partial \Omega$, and to be able to determine when such a distribution is in $h^{p}\left(\mathbf{R}^{n}\right)$. While several sufficient conditions are known in general (see [S2], 5.18), there is one case in which the necessary and sufficient conditions are well understood, which is the case where $\partial \Omega$ consists of a single point, i.e. when

$$
\Omega=\mathbf{R}_{+}=\{x \in \mathbf{R}: x>0\} .
$$

In this case, we denote by $\mathcal{S}\left(\mathbf{R}_{+}\right)$the space of Schwartz functions with support in $\mathbf{R}_{+}$, and let $\mathcal{S}^{\prime}\left(\mathbf{R}_{+}\right)$be the dual space. The space $h_{r}^{p}\left(\mathbf{R}_{+}\right)$is the subspace of $\mathcal{S}^{\prime}\left(\mathbf{R}_{+}\right)$consisting of restrictions of elements of $h^{p}(\mathbf{R})$ to $\mathbf{R}_{+}$. As above, $h_{z}^{p}\left(\overline{\mathbf{R}_{+}}\right)$is the space of elements of $h^{p}(\mathbf{R})$ which are supported on $\overline{\mathbf{R}_{+}}$.

Lemma 6.7. Let $p=1 /(1+N), N=0,1,2, \ldots$ Then there exists a distribution $f \in h_{r}^{p}\left(\mathbf{R}_{+}\right)$such that for every $g \in h_{z}^{p}\left(\overline{\mathbf{R}_{+}}\right)$,

$$
\left.g\right|_{\mathbf{R}_{+}} \neq f
$$

in $\mathcal{S}^{\prime}\left(\mathbf{R}_{+}\right)$.

Proof of lemma. We will give two examples, which are essentially two versions of the same example.

For the first example, we take

$$
f_{1}(x)=\frac{d^{N+1}}{d x^{N+1}}\left[\left(\log \frac{1}{x}\right)^{-1 / p} \eta(x)\right],
$$

defined for $x>0$, where $\eta$ is a smooth function satisfying $\eta(x)=1$ for $0<x \leq 1 / 2$ and $\eta(x)=0$ when $x \geq 3 / 4$. Being the derivative of a smooth function on $\mathbf{R}_{+}$with bounded support, $f_{1}$ defines a distribution in $\mathcal{S}^{\prime}\left(\mathbf{R}_{+}\right)$. Note that since $N+1=1 / p$,

$$
f_{1}(x) \approx\left(\log \frac{1}{x}\right)^{-1 / p-1} x^{-1 / p} \eta(x)
$$


as $x \rightarrow 0$, so $f_{1}$ is not a distribution in $\mathcal{S}^{\prime}(\mathbf{R})$. To extend $f_{1}$ to such a distribution, we let

and define

$$
h(x)= \begin{cases}\left(\log \frac{1}{x}\right)^{-1 / p} \eta(x) & \text { if } x \geq 0 \\ 0 & \text { if } x \leq 0\end{cases}
$$

$$
F_{1}=\frac{d^{N+1}}{d x^{N+1}} h
$$

in the sense of distributions. Then $F_{1} \in \mathcal{S}^{\prime}(\mathbf{R})$ and $\left.F_{1}\right|_{\mathbf{R}_{+}}=f_{1}$.

We build the second example by means of an atomic decomposition, analogous to the one constructed in the proof of Proposition 6.4. For $j \geq 1$, set

and

$$
\begin{gathered}
I_{j}=\left[2^{-j}, 2^{-j+1}\right], \\
\lambda_{j}=\frac{1}{j^{1+1 / p}},
\end{gathered}
$$

Let

$$
a_{j}=2^{j / p} \chi_{I_{j}} .
$$

$$
f_{2}=\sum_{j=1}^{\infty} \lambda_{j} a_{j}
$$

Again $f_{2}$ defines a distribution in $\mathcal{S}^{\prime}\left(\mathbf{R}_{+}\right)$, and for $x \approx 2^{-j}$,

$$
f_{2}(x) \approx \frac{1}{j^{1+1 / p}} 2^{j / p} \approx\left(\log \frac{1}{x}\right)^{-1 / p-1} x^{-1 / p}
$$

To extend $f_{2}$ to a distribution in $\mathcal{S}^{\prime}(\mathbf{R})$, we define distributions $\tau_{j}$ by

$$
\left\langle\tau_{j}, \varphi\right\rangle=\lambda_{j} \int a_{j}(x)\left[\varphi(x)-\sum_{k=0}^{N-1} \frac{1}{k !} \frac{d^{k} \varphi}{d x^{k}}(0) x^{k}\right] d x
$$

for every $\varphi \in \mathcal{S}(\mathbf{R})$. Since

$$
\begin{aligned}
\left|\left\langle\tau_{j}, \varphi\right\rangle\right| & =\lambda_{j} 2^{j / p}\left|\int_{I_{j}}\left[\varphi(x)-\sum_{k=0}^{N-1} \frac{1}{k !} \frac{d^{k}}{d x^{k}} \varphi(0) x^{k}\right] d x\right| \\
& \leq \lambda_{j} 2^{j / p}\|\varphi\|_{\mathcal{C}^{N}} \int_{I_{j}} x^{N} d x \\
& \leq C \frac{1}{j^{1+1 / p}} 2^{j(1 / p-N-1)}\|\varphi\|_{\mathcal{C}^{N}} \\
& =C \frac{1}{j^{1+1 / p}}\|\varphi\|_{\mathcal{C}^{N}}
\end{aligned}
$$

we can define a distribution $F_{2} \in \mathcal{S}^{\prime}(\mathbf{R})$ by

$$
F_{2}=\sum_{j=1}^{\infty} \tau_{j}
$$

Again we have that

$$
\left.F_{2}\right|_{\mathbf{R}_{+}}=\left.\sum_{j=1}^{\infty} \tau_{j}\right|_{\mathbf{R}_{+}}=\sum_{j=1}^{\infty} \lambda_{j} a_{j}=f_{2} .
$$

We shall prove the result for $f_{2}$; there are parallel arguments for $f_{1}$. 
Claim 6.8. $f_{2}$ belongs to $h_{r}^{p}\left(\mathbf{R}_{+}\right)$.

This is via the atomic decomposition which defines $f_{2}$.

Claim 6.9. The distribution $F_{2}$ does not belong to $h^{p}(\mathbf{R})$, since the local grand maximal function satisfies

$$
m\left(F_{2}\right)(x) \approx\left(\log \frac{1}{x}\right)^{-1 / p} x^{-1 / p}
$$

for $0<x<1 / 2$.

To get the lower bound for $m\left(F_{2}\right)$, take $0<x<1 / 2$. We will use a normalized bump function $\phi_{x}$ supported in the interval $(-x, 3 x)$, with $\phi_{x} \geq 0$ on $(x, 3 x)$ and

$$
\phi_{x}(y)=\frac{y^{N}}{N !(2 x)^{1+N}} \text { for } y \in[0, x] .
$$

Note that this is consistent with the condition

$$
\left|D^{\alpha}\left(\phi_{x}\right)\right| \leq(2 x)^{-1-|\alpha|}
$$

for $|\alpha| \leq N+1$. Furthermore,

$$
\frac{d^{k} \phi_{x}}{d y^{k}}(0)=0
$$

for all $k \leq N-1$. Thus $\left\langle F_{2}, \phi_{x}\right\rangle=\sum \lambda_{j} \int a_{j} \phi_{x}$ and we can proceed as in the proof of Proposition 6.4 to get

$$
\begin{aligned}
\left\langle F_{2}, \phi_{x}\right\rangle & \geq \sum_{I_{j} \subset[0, x]} \lambda_{j} \int_{I_{j}} a_{j}(y) \phi_{x}(y) d y \\
& =\frac{1}{N !(2 x)^{1+N}} \sum_{I_{j} \subset[0, x]} \lambda_{j} \int_{I_{j}} a_{j}(y) y^{N} d y \\
& =\frac{2^{N+1}-1}{(N+1) !(2 x)^{1+N}} \sum_{I_{j} \subset[0, x]} \lambda_{j} 2^{j(1 / p-N-1)} \\
& \geq C_{N} x^{-1-N} \sum_{j \geq-\log _{2} x} j^{-1 / p-1} \\
& \approx x^{-1 / p}\left(\log \frac{1}{x}\right)^{-1 / p}
\end{aligned}
$$

This gives the lower bound on the maximal function of $F_{2}$.

Now we will prove the upper bound on the maximal function of $F_{2}$. Take $0<x<$ $1 / 2$, and let $\varphi_{t}^{x}$ be a bump function supported in a ball of radius $t \leq 1$ containing $x$.

Suppose first that $t \geq x / 4$. If $2^{-j}>6 t \geq x+2 t$, then $\operatorname{supp}\left(\varphi_{t}^{x}\right) \cap I_{j}=\emptyset$, and we have

$$
\begin{aligned}
\left|\left\langle\tau_{j}, \varphi_{t}^{x}\right\rangle\right| & =\left|\lambda_{j} 2^{j / p} \sum_{k \leq N-1} \frac{1}{k !} d^{k} \varphi_{t}^{x}(0) \int_{I_{j}} x^{k} d x\right| \\
& \leq C \lambda_{j} \sum_{k \leq N-1} \frac{1}{k !} t^{-1-k} 2^{j(1 / p-1-k)},
\end{aligned}
$$


while otherwise we use the estimate

$$
\left|\left\langle\tau_{j}, \varphi_{t}^{x}\right\rangle\right| \leq C \lambda_{j}\left\|\varphi_{t}^{x}\right\|_{\mathcal{C}^{N}} \leq C \lambda_{j} t^{-1-N} .
$$

Thus

$$
\begin{aligned}
\left|\left\langle F_{2}, \varphi_{t}^{x}\right\rangle\right| \leq & C \sum_{k \leq N-1} \frac{1}{k !} t^{-1-k} \sum_{j<-A \log t} \frac{2^{j(N-k)}}{j^{1+1 / p}} \\
& +C t^{-1-N} \sum_{j \geq-A \log t} \frac{1}{j^{1+1 / p}} \\
\leq & C \sum_{k \leq N-1} \frac{1}{k !} t^{-1-k} t^{-(N-k)}(-\log t)^{-1 / p} \\
& +C t^{-1-N}(-\log t)^{-1 / p} \\
\leq & C t^{-1 / p}\left(\log \frac{1}{t}\right)^{-1 / p} \\
\leq & C x^{-1 / p}\left(\log \frac{1}{x}\right)^{-1 / p}
\end{aligned}
$$

since $(x / t)^{1 / p}(\log x / \log t)^{1 / p}$ remains bounded when $x \ll t$.

If $t \leq x / 4$, we have that $\varphi_{t}^{x}$ vanishes in a neighborhood of 0 , and

$$
\begin{aligned}
\left|\left\langle F_{2}, \varphi_{t}^{x}\right\rangle\right| & \leq C \sum_{j \leq-A \log x} \lambda_{j}\left|\int a_{j} \varphi_{t}^{x}\right| \\
& \leq C \sum_{j \leq-A \log x} \frac{1}{j^{1+1 / p}} 2^{j / p}\left\|\varphi_{t}^{x}\right\|_{L^{1}} \\
& \leq C x^{-1 / p}\left(\log \frac{1}{x}\right)^{-1 / p} .
\end{aligned}
$$

Thus we have shown that for $0<x<1 / 2$,

$$
m\left(F_{2}\right)(x) \approx x^{-1 / p}\left(\log \frac{1}{x}\right)^{-1 / p} .
$$

Claim 6.10. Then there is no distribution $g \in h^{p}(\mathbf{R})$ with $g=0$ on $\mathbf{R}_{-}$and $g=f_{2}$ on $\mathbf{R}_{+}$.

Recall that $F_{2}$ is supported on $\overline{\mathbf{R}_{+}}$and $F_{2}=f_{2}$ on $\mathbf{R}_{+}$. Thus if $g$ is any distribution in $\mathcal{S}^{\prime}(\mathbf{R})$ with $g=0$ on $\mathbf{R}_{-}$and $g=f_{2}$ on $\mathbf{R}_{+}$, then $g-F_{2}$ is supported on $\{0\}$. Since tempered distributions are of finite order, we can write (see [R], Theorem 6.25)

$$
g-F_{2}=\sum_{k=0}^{K} c_{k} \delta^{(k)}(0),
$$

where $\delta^{(k)}(0)$ denotes the $k$-th derivative of the delta function at 0 . We may assume $c_{K} \neq 0$. Then if $K \geq N$, we have

$$
m\left(g-F_{2}\right) \geq C|x|^{-N-1}
$$


as $x \rightarrow 0$, and so by the upper bounds on $m(F)$,

$$
m(g) \geq C|x|^{-N-1}=C|x|^{-1 / p}
$$

for $x$ small, and $m(g) \notin L^{p}(\mathbf{R})$.

On the other hand, if $K<N$, then

$$
m\left(g-F_{2}\right) \leq C|x|^{-N}=C|x|^{1-1 / p}
$$

as $x \rightarrow 0$, so by the lower bounds on $m\left(F_{2}\right)$,

$$
m(g) \geq C|x|^{-1 / p}\left(\log \frac{1}{x}\right)^{-1 / p}
$$

for $x$ small, and again $m(g) \notin L^{p}(\mathbf{R})$.

This completes the proof of the lemma.

\section{REFERENCES}

[ADN] S. Agmon, A. Douglis, L. Nirenberg, Estimates near the boundary for solutions of elliptic partial differential equations satisfying general boundary conditions, I, Comm. Pure Applied Math. 12 (1959), 623-727. MR 23:A2610

[C] D.-C. Chang, The dual of Hardy spaces on a bounded domain in $\mathbf{R}^{n}$, Forum Math. 6 No. 1 (1994), 65-81. MR 95b:42022

[CKS] D.C. Chang, S. G. Krantz, and E. M. Stein, $H^{p}$ Theory on a smooth domain in $\mathbf{R}^{N}$ and elliptic boundary value problems, J. Funct. Anal. 114, No. 2 (1993), 286-347. MR 94j:46032

[D] G. Dafni, Hardy Spaces on Strongly Pseudoconvex Domains in $\mathbf{C}^{n}$ and Domains of Finite Type in $\mathbf{C}^{2}$, Ph.D. Thesis, Princeton University, 1993.

[D2] G. Dafni, Distributions supported in a hypersurface and local $h^{p}$, Proc. Amer. Math. Soc. 126 (1998), 2933-2943. CMP 98:16

[F] G. B. Folland, Introduction to Partial Differential Equations, Math. Notes 17, Princeton Univ. Press, Princeton, New Jersey, 1976. MR 58:29031

[FS] C. Fefferman and E. M. Stein, $H^{p}$ spaces of several variables, Acta Math. 129 (1972), 137-193. MR 56:6263

[G] D. Goldberg, A local version of real Hardy spaces, Duke Math. J. 46 (1979), 27-42. MR 80h: 46052

[GS] P. C. Greiner and E. M. Stein, Estimates for the $\bar{\partial}-$ Neumann Problem, Math. Notes 19, Princeton Univ. Press, Princeton, New Jersey, 1977. MR 58:17218

[J] P. W. Jones, Extension theorems for BMO, Indiana Univ. Math. J. bf 29, No. 1 (1980), 41-66. MR 81b:42047

[JSW] A. Jonsson, P. Sjögren, and H. Wallin, Hardy and Lipschitz spaces on subsets of $\mathbf{R}^{n}$, Studia Math. 80, No. 2 (1984), 141-166. MR 87b:46022

[KL] S. G. Krantz, S. Y. Li, Elliptic boundary value problems for the inhomogeneous Laplace equation on bounded domains, preprint.

[M] A. Miyachi, $H^{p}$ spaces over open subsets of $\mathbf{R}^{n}$, Studia Math. 95, No. 3 (1990), 205-228. MR 91m:42022

[R] W. Rudin, Functional Analysis, Second Edition, McGraw-Hill, New York, 1991. MR 92k:46001

[Ry] V. S. Rychkov, Intrinsic characterizations of distribution spaces on domains, Studia Math. 127 (1998), 227-298. CMP 98:06

[S1] E. M. Stein, Singular Integrals and Differentiability Properties of Functions, Princeton Univ. Press, Princeton, New Jersey, 1970. MR 44:7280 
[S2] E. M. Stein, Harmonic Analysis: Real-Variable Methods, Orthogonality, and Oscillatory Integrals, Princeton Univ. Press, Princeton, New Jersey, 1993. MR 95c:42002

[SW] E. M. Stein and G. Weiss, Introduction to Fourier Analysis on Euclidean Spaces, Princeton Univ. Press, Princeton, New Jersey, 1971. MR 46:4102

Department of Mathematics, University of Maryland, College Park, Maryland 20742 Current address: Department of Mathematics, Georgetown University, Washingon, DC 20057

E-mail address: drc@math.umd.edu

Department of Mathematics \& Statistics, Concordia University, Montreal, Quebec H3G-1M8, CANADA

E-mail address: gdafni@discrete.concordia.ca

Department of Mathematics, Princeton University, Princeton, New Jersey 08544

E-mail address: stein@math.princeton.edu 